\title{
Kinematic modelling of disk galaxies ${ }^{\star}$
}

\section{A case-study of symmetrically warped galaxy disks}

\author{
G. I. G. Józsa
}

\begin{abstract}
Argelander Institute for Astronomy (AIfA), Univ. Bonn, Auf dem Hügel 71, 53121 Bonn, Germany
e-mail: gjozsa@astro.uni-bonn.de
\end{abstract}

Received 2 August 2006 / Accepted 2 March 2007

\section{ABSTRACT}

\begin{abstract}
This is the second of a series of papers in which the kinematics of disk galaxies is studied by analysing spectroscopic data. Making use of the methods presented in Paper I, this work focuses on the large-scale distribution of disk and Dark Matter halo material by analysing the structure of warped galaxies. H I observations of three disk galaxies showing regular large-scale ("grand-design") warps are presented and their structure is analysed. The kinematics and geometry of the galaxies are modelled by fitting a tilted-ring model directly to the data cube, thereby avoiding problems due to orbit crowding or beam smearing.

The galaxies follow the previously known "rules for the behaviour of warps". Furthermore, at large radii the H I disk settles into a relatively coplanar disk that is inclined with respect to the inner one. With increasing radius, the measured rotation velocity shows a rise. In one of the cases, NGC 5204, the rotation curve shows a jump at the transition radius. While the best fits in the other two cases also suggest that such a jump is present, the change in rotation velocity is compatible with a gradual rise. In two cases, the surface density of the warped $\mathrm{H}$ I layer shows a clear drop at the transition radius and decreases only very slowly further out, while this feature is not as clear for UGC 3580. A literature search shows that these properties are shared by a number of other galaxies. In all cases, an additional, slowly rotating gas layer is found to be present.

The change in the rotation velocity and the remarkable geometry of the disks together indicate that the radius at which the warp starts marks the transition between two dynamical regimes, an inner one governed by the stellar disk and an outer one determined by the Dark Matter halo.
\end{abstract}

Key words. galaxies: kinematics and dynamics - galaxies: structure - galaxies: individual: NGC 2541 galaxies: individual: UGC 3580 - galaxies: individual: NGC 5204

\section{Introduction}

Warps in the disks of spiral galaxies are ubiquitous. If traced sufficiently well beyond the optical radius, independent of the environment, galaxy disks bend away from the plane defined by the inner portion of the disk (García-Ruiz et al. 2002).

Today, warps are particularly interesting in the context of cosmology on galaxy scales. Usually they are interpreted as the result of an external torque exerted on the disk (e.g. by a misaligned and flattened outer halo as is often seen in cosmological simulations, Sharma \& Steinmetz 2005, see below), possibly being the visible part of "excitations of the oscillation modes of quasi-equilibrium halos" (Gao \& White 2006). Once fully understood, determination of their properties can serve to put constraints on the large-scale structure and evolution of galaxies and their Dark Matter (DM) halos, and hence on galaxy formation scenarios. This second paper in a series presents a detailed analysis of the large-scale morphological and kinematical structure of prototype warped galaxies. While the literature does not provide a uniform picture, most recent studies (García-Ruiz et al. 2002; Sánchez-Saavedra et al. 2003) indicate that warp shapes are dominated by the (anti-) symmetric S-shaped "grand-design" warp, the projection of the galaxy resembling an $\mathrm{S}$ or an integral sign.

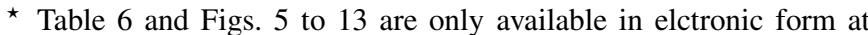
http: //www . aanda.org
A few rules for the behaviour of such (antisymmetric) warps exist although they are not strictly followed by all galaxies, the rules were proposed by Briggs (1990). Of these the most important ones are: i) Galactic (H I-)disks are planar within $R_{25}$. The kinematical minor axis is straight inside a transition radius $R_{\mathrm{tr}} \approx R_{\mathrm{Ho}}$. (Rule 1 in Briggs 1990.) This implies that, if the inner galactic disk is precessing, the disk keeps the same precession frequency inside $R_{\mathrm{tr}}$, where a warp is already occurring. ii) Warps change character at $R_{\text {tr }}$ (Rule 2 in Briggs 1990). iii) For radii larger than $R_{\mathrm{Ho}}\left(\approx R_{\mathrm{tr}}\right)$, the kinematical minor axis progresses in the direction of the galactic rotation (Rule 3 in Briggs 1990), implying the onset of a characteristic differential precession pattern at radii $\gtrsim R_{\text {tr }}$ ). iv) A unique reference frame exists in which the kinematical minor axis exhibits two straight regimes, one inside $R_{\mathrm{tr}}$ and one beyond $R_{\mathrm{tr}}$ (Rule 4 in Briggs 1990 . One thus finds an outer regime, in which co-precession is also present, cf. Revaz \& Pfenniger 2001). v) Warps are common and occur in all environments (Rogstad et al. 1974; Bosma 1978). The environment plays a role insofar as the amplitude of warps is higher (Reshetnikov \& Combes 1998; Schwarzkopf \& Dettmar 2001; Sánchez-Saavedra et al. 2003) and warps are more asymmetric in dense environments (García-Ruiz et al. 2002). vi) No warp exists that shows clear differences of amplitude in the stellar and the gaseous component (Cox et al. 1996, but see also Florido et al. 1991). While the rules i), ii), iii), v), vi) are commonly accepted, iv) is a controversial point that has been put down to projection effects (Briggs \& Kamphuis 1991). 
Recent studies of Sánchez-Salcedo (2004, 2006) indicate that the intergalactic medium in the form of magnetic fields (Battaner et al. 1990b,a; Battaner \& Jimenez-Vicente 1998) or intergalactic gas (Kahn \& Woltjer 1959; López-Corredoira et al. 2002) can be ruled out as an interaction partner that enables warp formation. The possibility of the growth of self-inflicted bending instabilities is discussed by Revaz \& Pfenniger (2004). Such models have the drawback that their warp amplitude is too small to explain the numerous isolated galaxies with large symmetric warps and that they partly violate rule iii).

Tidal interactions with small companion galaxies as a possible origin of warps are an attractive proposal. More and more small stellar systems in the environment of seemingly isolated warped galaxies are detected due to the improvement in instrumental sensitivity (e.g. Shang et al. 1998). In denser environments more interaction partners are present, hence that warps are stronger in denser environments has a natural explanation. Warp excitation by tidal interaction has been examined in a number of studies (Burke 1957; Kerr 1957; Elwert \& Hablick 1965; Avner \& King 1967; Weinberg 1998; Brada \& Milgrom 2000; Bailin 2003), but only recently could it be shown that the shape and the amplitude of the rather irregular Milky Way warp can be qualitatively reproduced in a simulation of tidal interaction with the Large Magellanic Cloud (Weinberg \& Blitz 2006). In the same study it was shown that beneath the "grand-design" $m=1$ vertical distortion, other modes are excited so that a warp generated by tidal interaction will in general have an asymmetric appearance. While certainly explaining the fact that warps are more asymmetric in denser environments, the high frequency of regular grand-design warps cannot be explained by a tidal interaction scenario alone.

Bending modes in the presence or without the presence of a stiff DM halo in order to ensure a long-lived warping of the disk have been studied extensively (Lynden-Bell 1965; Hunter \& Toomre 1969; Binney 1978; Tubbs \& Sanders 1979; Dekel \& Shlosman 1983; Toomre 1983; Sparke 1984, 1985; Sparke \& Casertano 1988; Kuijken 1991). All these models fail to reproduce rule iii). Proposals that warps could be temporary features that reflect the settling into a bending mode or a modifiedtilt mode (Hofner \& Sparke 1994; see also Christodoulou \& Tohline 1986) could be excluded when the halo was not longer regarded as a stiff entity but as a live (reactive) system (Nelson \& Tremaine 1995; Dubinski \& Kuijken 1995; Binney et al. 1998) that quickly aligns with the disk in the region of the disk. It is nevertheless an issue of discussion whether for a realistic setup bending modes of a coupled disk-halo system might exist, a point stressed by Binney et al. (1998). They observe that, while in their simulations the warp winds up rapidly, the "warping energy" is still preserved. For the right setup of an initial model, a simulation could possibly converge to a steady-state "mode", involving disk and (flattened) DM halo.

Being aware of the problems with bending modes in the presence of a (static) DM halo, Ostriker \& Binney (1989) and Binney (1992) demonstrate that secondary cosmic infall on the halo, reorientating it steadily, might be responsible for warp formation. Various simplified computer simulations in which cosmic infall was taken into account have been performed. Debattista \& Sellwood (1999) find a surprisingly realistic warp by simulating the evolution of a initially flat disk in a DM halo with inclined spin axis, successfully reproducing Briggs' (1990) rules. Following up an earlier experiment by Jiang \& Binney (1999), Shen \& Sellwood (2006) used an improved setup to simulate the evolution of a disk-halo system into which halo material was injected into an inclined torus. The torus was set up to be non-reactive, thereby acting as an external agent. They were able to produce a transient, but quite long-lived warp fulfilling the three accepted rules i)-iii). They made a testable prediction: at a large radius the sign of differential precession (iii) would be reversed, with the "twist radius" depending on the position and the mass of the torus. After removal of the torus, the produced warp dampens extremely slowly by an (rather ineffective) exchange of angular momentum between the misaligned inner and outer portions of the flattened DM halo. Such long-lived misalignment between inner and outer portions of DM halos has been found to evolve in a cosmological concordance scenario (Bailin et al. 2005) and is indeed reminiscent of a steady-state bending structure of the disk-inner halo system and an outer flattened halo, a bending mode including disk and an intrinsically misaligned halo.

In this paper we present the study of the kinematical warp structure of three galaxies, selected to show the prototype of symmetric grand-design warps of intermediate up to large amplitudes. The goal of this study is to scrutinise the rules formulated by Briggs (1990), to check their validity, and furthermore to possibly constrain the general structure of grand-design warps further, making use of the higher sensitivity provided by today's telescopes and improved analysis methods.

The paper is laid out as follows: In Sect. 2 the selection and global properties of the observed galaxies are described. Section 3 presents details of the H I synthesis observations conducted with the Westerbork Synthesis Radio Telescope and the data reduction. In Sect. 4 the data analysis by means of a new technique to perform a tilted-ring modelling is described, the results of which are shown in Sect. 6, and Sect. 5 briefly describes the distance estimation using the fitting results. In Sect. 7 the fitting results are critically reviewed and the influence of violating systematic effects is taken into account. Section 8 contains a discussion of the final results in view of possible mechanisms that drive warping. Supplementary figures and tables not essential for a reading of this paper are placed in the online publication, and more details on data reduction and analysis can be found in Józsa (2006).

\section{The galaxies studied}

The selection of suitable candidates was dominated by the desire to choose prototype grand-design warps that do not show direct signs of tidal interaction or other peculiarities in their H I structure. The galaxies were selected to be classified as non-barred in the RC3 (de Vaucouleurs et al. 1991) and not to be members of a galaxy cluster. In order to be able to get an impression whether a galaxy would serve for the intended purpose, the WHISP (van der Hulst et al. 2001) database, which provides H I total intensity maps and, equally important, velocity maps of a large sample of galaxies, was consulted in order to select the spiral galaxies NGC 2541, UGC 3580, and NGC 5204 as suitable objects.

NGC 2541 is a well-known smaller late-type spiral galaxy in the NGC 2841 galaxy group, which contains 7 larger spiral galaxies in total. While listed in the RC3 as a non-barred galaxy, it is classified as showing a bar in the PGC (Paturel 1989). Chapelon et al. (1999) and Cabrera-Lavers \& Garzón (2004) show in their photometric analyses that this galaxy has a central isophotal twist that they interprete as a bar with a diameter of $\approx 38^{\prime \prime}$. Analysing photometric observations, Coccato et al. (2004) do not confirm the presence of a bar, but find a strong velocity gradient along the minor axis of the galaxy in their long-slit data. Using Fabry-Pérot spectroscopy, Garrido et al. (2004) found a characteristic kinematical feature at the end of the 
assumed bar, the extent of which they estimate to be $30^{\prime \prime}$ in projection. Either NGC 2541 possesses a small bar or an inclined inner disk at the innermost radii. It is a convenient coincidence that NGC 2541 is part of the HST key project to measure the Hubble constant, and it has been used as a Tully-Fisher calibrator. Its distance has been measured with great accuracy using the Cepheid period-luminosity relation (Ferrarese et al. 1998; Freedman et al. 2001).

UGC 3580 is an early-type spiral of similar size as NGC 2541. This galaxy is poorly studied and has no obvious companions. While the RC3 (de Vaucouleurs et al. 1991) classifies this galaxy as being unbarred, in the PGC (Paturel 1989) it is classified as containing a central bar.

NGC 5204 is a rather small galaxy of Magellanic type. It entered this study because of it's extraordinary large and symmetric warp. The galaxy is supposed to lie at the border of the M101 group (Karachentsev et al. 1994, 2003) but has no obvious companions or interaction partners. The distance of $4.6 \mathrm{Mpc}$, determined by Karachentsev et al. (2003) (compare to 4.95 Mpc found by Swaters \& Balcells 2002), is rather low for a member of the M101 group (see also Table 1). Because of its symmetry, it entered several kinematical and dynamical studies (Sicotte et al. 1996; Sicotte \& Carignan 1997; Swaters et al. 2000; Swaters 2001; Swaters et al. 2003).

Basic properties of the galaxies, derived in this work or taken from literature, are listed in Table 1.

\section{H I observations and data reduction}

The galaxies were observed in the $\mathrm{H}$ I emission line in-between December 2002 and May 2003 with the upgraded WSRT: NGC 2541 and UGC 3580 for $1 \times 12$ h, NGC 5204 for $2 \times 12 \mathrm{~h}$. Details of the observations are listed in Table 2.

The data underwent a standard data reduction with the Miriad software package (Sault et al. 1995). After the initial calibration, editing, and second-order continuum subtraction, a self calibration of the subtracted continuum data was performed to correct the small phase- and - if necessary - amplitude errors of the broad-band gain. A second continuum removal was performed by subtracting the inversion of the obtained continuum model from the visibilities and subsequent fitting of the continuum residuals to first or (if necessary) second order. After inversion of the line data using various weighting schemes (see Table 2), the data were CLEANed iteratively determining more and more accurate CLEAN regions and lower cutoff parameters. The iteration was stopped at a cutoff of one third to half of the rms noise in the channel maps. For the subsequent analysis, two data cubes were used per galaxy, a high-resolution data cube (see Fig. 6 in the online version of this paper) using uniform weighting at full resolution, and a low-resolution data cube (Fig. 5 in the online version), decreasing the velocity resolution by a factor of two and using only baselines with a size of maximal $6.5 \mathrm{k} \lambda_{\mathrm{HI}}$ $\left(\lambda_{\mathrm{H} \text { I }} \approx 21.1 \mathrm{~cm}\right)$ and a robust weighting of 0.4 . Total intensity maps and first-moment maps were generated by first masking the cubes with the finally applied clean masks. Also in the following analysis it was refrained from generating velocity fields more suitable to representing the mid-plane kinematics of the galaxies like Gaussian or Gaussian Hermite velocity fields (introducing more parameters to characterise spectra), since the the velocity fields were mainly used to check the quality of the tilted-ring models as derived directly from the data cubes (Sect. 4) themselves. As the tilted-ring model was fitted to global spectra, a velocity field better representing the global aspects of the single spectra with one parameter was preferred. The resulting maps are shown in Fig. 2 and in Fig. 7 and 8 in the online version of this paper.

For the calculation of the total H I flux in Table 1, a primary beam correction was applied, while for the later work uncorrected maps were used in order to keep the noise distributed uniformly over the channel maps. A more detailed description of the data reduction can be found in Józsa (2006).

\section{Tilted-ring modelling}

The HI kinematics of the observed galaxies were parametrised by means of the tilted-ring model (Rogstad et al. 1974) employing the newly developed software "TiRiFiC" (see Józsa et al. 2007a, hereafter Paper I). TiRiFiC automatically fits synthetic observations of a galaxy parametrised in terms of the tilted-ring model directly to the data cube optimising the model parameters via $\chi^{2}$ minimisation. This approach significantly mitigates the problems due to beam smearing and avoids problems due to ambiguities caused by the line-of-sight intersecting the disk twice or more, which might occur when analysing velocity fields, especially in the case of warped galaxies.

In order to optimise the parameters, the golden-section approach described in Paper I was applied. Initial guesses for the parametrisation of the galaxies were taken from the literature (Sicotte \& Carignan 1997) or estimated from velocity fields. As discussed in Paper I, a few iterations on the initial values for TiRiFiC were necessary to obtain stable results for all parameters. This approach is necessary, because for (only) a few parameters the $\chi^{2}$ minimisation algorithm lacks efficiency in terms of scanning the parameter space. In Paper I it is shown that this technique of stirring up the parameters in order to enable the repeated search for the $\chi^{2}$ minimum in slightly different regions in parameter space quickly leads to the best fit.

For each galaxy, the low-resolution cube was analysed first, varying the number of point sources between $1.7 \times 10^{6}$ and $4.0 \times 10^{6}$. The centre of the galaxy, the global velocity dispersion, and the scaleheight were kept as free parameters but were not allowed to vary independently from ring to ring (in case of the velocity dispersion, the user has no other choice). The symmetry of the galaxies allowed for this restriction (see Sect. 7). For each ring, the surface brightness, the orientational parameters, and the rotation velocity were varied independently. For the estimation of the errors, a traditional, crude method was applied by comparing individual fits to the approaching and the receding side of the galaxies, and. if necessary, by visual inspection of the data cubes varying individual parameters. The errors of the central position and the systemic velocity of the galaxy had to be estimated without a cross check, but these global values proved to be very robust from fit to fit and changes were well inside the observational beam and the velocity resolution, respectively, less than $4^{\prime \prime}$ or $1 \mathrm{~km} \mathrm{~s}^{-1}$. The errors that may safely be adopted here can be estimated to be less than $12^{\prime \prime}$ for the position of the kinematical centre and less than $2 \mathrm{~km} \mathrm{~s}^{-1}$ for the systemic velocity.

In all cases, the models (triangles in Fig. 1, red in online version) showed a central tilt of the disk in the innermost few tens of arcseconds. Whether this is a real feature cannot be decided. Alternatively, TiRiFiC could fail for these inner few rings due to the small number of independent data points (a statistical error, see also Paper I) or due to non-circular motions that are confused for a change in the orientational parameters (a systematic error). Therefore, for each galaxy, another model was fit assuming a flat disk in the the inner part of the galaxy. The disk was forced to retain equal but variable orientational parameters inside a radius, outside of which the outer warp starts, i.e. well inside the radius 
Table 1. Basic properties of the examined galaxies. (1) de Vaucouleurs et al. (1991); (2) Freedman et al. (2001); (3) This work; (4) NED; (5) LEDA (Paturel et al. 2003).

\begin{tabular}{|c|c|c|c|c|c|}
\hline Descr. & Par. & NGC 2541 & $\overline{\text { UGC } 3580}$ & NGC 5204 & $\overline{\text { Ref. }}$ \\
\hline Classification of galaxy & Type & SA(s)cd & SA(s)a & SA(s)m & (1) \\
\hline Right Ascension (J2000) (NED) & RA & $08^{\mathrm{h}} 14^{\mathrm{m}} 40^{\mathrm{s}} 07$ & $06^{\mathrm{h}} 55^{\mathrm{m}} 30.86$ & $13^{\mathrm{h}} 29^{\mathrm{m}} 36^{\mathrm{s}} 51$ & (4) \\
\hline Declination (J2000) (NED) & Dec & $+49^{\circ} 03^{\prime} 41^{\prime \prime} 2$ & $+69^{\circ} 33^{\prime} 47^{\prime \prime} 0$ & $+58^{\circ} 25^{\prime} 07^{\prime \prime} 4$ & (4) \\
\hline Optical heliocentric systemic velocity $\left(\mathrm{km} \mathrm{s}^{-1}\right)$ & $V_{\text {sys }}$ & $559.5 \pm 2.0$ & $1200.1 \pm 2.0$ & $200.8 \pm 2.0$ & (3) \\
\hline Distance of object (Mpc) & $D^{\infty}$ & $11.2 \pm 0.8$ & $15.1 \pm 3.5$ & $4.1 \pm 1.0$ & $(2,3)$ \\
\hline Scale between distance on sky and true distance $\left(" \mathrm{pc}^{-1}\right)$. & $s c$ & $54.4 \pm 3.9$ & $73 \pm 17$ & $20.0 \pm 4.8$ & (3) \\
\hline Apparent $B$-band magnitude (mag). & $m_{\mathrm{B}}$ & $12.36 \pm 0.34$ & $13.87 \pm 0.35$ & $11.71 \pm 0.12$ & (5) \\
\hline Apparent $I$-band magnitude (mag). & $m_{\mathrm{I}}$ & $10.86 \pm 0.06$ & $11.55 \pm 0.08$ & $9.79 \pm 0.10$ & (5) \\
\hline Extinction-corrected apparent $B$-band magnitude (mag). & $m_{\mathrm{B}}^{\mathrm{c}}$ & $11.60 \pm 0.34$ & $13.34 \pm 0.35$ & $11.30 \pm 0.12$ & (4) \\
\hline Extinction-corrected apparent $I$-band magnitude (mag). & $m_{\mathrm{I}}^{\mathrm{c}}$ & $10.57 \pm 0.07$ & $11.25 \pm 0.09$ & $9.71 \pm 0.10$ & (3) \\
\hline Absolute $B$-band magnitude (mag). & $M_{\mathrm{B}}$ & $-18.6 \pm 0.4$ & $-17.6 \pm 0.6$ & $-16.8 \pm 0.5$ & (3) \\
\hline Absolute $I$-band magnitude (mag). & $M_{\mathrm{I}}$ & $-19.7 \pm 0.2$ & $-19.6 \pm 0.5$ & $-18.4 \pm 0.5$ & (3) \\
\hline$B$-band luminosity $\left(10^{9} L_{\odot}\right)$. & $L_{\mathrm{B}}$ & $4.5 \pm 1.6$ & $1.64 \pm 0.92$ & $0.81 \pm 0.39$ & (3) \\
\hline$I$-band luminosity $\left(10^{9} L_{\odot}\right)$ & $L_{\mathrm{I}}$ & $3.20 \pm 0.50$ & $3.1 \pm 1.5$ & $0.96 \pm 0.46$ & (3) \\
\hline Total H I flux (Jy km s$\left.{ }^{-1}\right)$. & $F_{\mathrm{HI}}$ & $142 \pm 15$ & $41.6 \pm 4.2$ & $135 \pm 14$ & (3) \\
\hline H I mass $\left(10^{9} M_{\odot}\right)$. & $M_{\mathrm{HI}}$ & $4.23 \pm 0.74$ & $2.2 \pm 1.1$ & $0.54 \pm 0.27$ & (3) \\
\hline$B$-band optical radius ("). & $r_{25}$ & $173 \pm 61$ & $97 \pm 28$ & $140 \pm 10$ & (5) \\
\hline H I radius ("). & $r_{\mathrm{HI}}$ & $420 \pm 30$ & $240 \pm 30$ & $240 \pm 30$ & (3) \\
\hline Terminal radius ("). & $r_{\mathrm{t}}$ & $590 \pm 30$ & $375 \pm 30$ & $420 \pm 30$ & (3) \\
\hline$B$-band optical radius (kpc). & $R_{25}$ & $9.39 \pm 0.67$ & $7.1 \pm 1.7$ & $2.82 \pm 0.66$ & (5) \\
\hline H I radius (kpc). & $R_{\mathrm{H} \mathrm{I}}$ & $22.9 \pm 1.7$ & $17.6 \pm 4.1$ & $4.8 \pm 1.2$ & (3) \\
\hline Terminal radius (kpc). & $R_{\mathrm{t}}$ & $32.1 \pm 2.3$ & $27.5 \pm 6.4$ & $8.4 \pm 2.0$ & (3) \\
\hline Rotation velocity at terminal radius $\left(\mathrm{km} \mathrm{s}^{-1}\right)$. & $V_{\mathrm{t}}$ & $127 \pm 25$ & $121 \pm 17$ & $105 \pm 25$ & (3) \\
\hline Rotation period at terminal radius (Gyr). & $t_{\mathrm{t}}$ & $1.55 \pm 0.04$ & $1.39 \pm 0.04$ & $0.49 \pm 0.03$ & (3) \\
\hline Dynamical mass $\left(10^{9} M_{\odot}\right)$. & $M_{\mathrm{dyn}}$ & $>121 \pm 49$ & $>94 \pm 34$ & $>22 \pm 12$ & (3) \\
\hline Ratio of H I mass and $B$-band luminosity $\left(M_{\odot} L_{\odot}^{-1}\right)$. & $\frac{M_{\mathrm{HI}}}{L_{\mathrm{B}}}$ & $0.9 \pm 0.2$ & $1.4 \pm 0.23$ & $0.7 \pm 0.07$ & (3) \\
\hline Ratio of H I mass and $I$-band luminosity $\left(M_{\odot} L_{\odot}^{-1}\right)$. & $\frac{M_{\mathrm{HI}}}{L_{\mathrm{I}}}$ & $1.3 \pm 0.2$ & $0.7 \pm 0.08$ & $0.6 \pm 0.06$ & (3) \\
\hline Ratio of dynamical mass and H I mass. & $\frac{M_{\mathrm{dyn}}}{M_{\mathrm{HI}}}$ & $>29 \pm 4$ & $>42 \pm 12$ & $>40 \pm 11$ & (3) \\
\hline Ratio of dynamical mass and $B$-band luminosity $\left(M_{\odot} L_{\odot}^{-1}\right)$. & $\frac{M_{\mathrm{dyn}}}{L_{\mathrm{B}}}$ & $>27 \pm 12$ & $>57 \pm 23$ & $>27 \pm 15$ & (3) \\
\hline Ratio of dynamical mass and $I$-band luminosity $\left(M_{\odot} L_{\odot}^{-1}\right)$. & $\frac{M_{\mathrm{dyn}}}{L_{\mathrm{I}}}$ & $>38 \pm 16$ & $>30 \pm 12$ & $>23 \pm 13$ & (3) \\
\hline
\end{tabular}

Table 2. Summary of observations and data reduction.

\begin{tabular}{|c|c|c|c|c|c|c|c|}
\hline \multicolumn{2}{|l|}{$\begin{array}{l}\text { Name of galaxy } \\
\text { Total on-source integration time (h) } \\
\text { Observation dates }\end{array}$} & \multicolumn{2}{|c|}{$\begin{array}{c}1 \times 12 \\
4-5 / 01 / 03\end{array}$} & \multicolumn{2}{|c|}{$\begin{array}{c}1 \times 12 \\
1-2 / 01 / 03\end{array}$} & \multicolumn{2}{|c|}{$\begin{array}{c}2 \times 12 \\
24-27 / 04 / 03\end{array}$} \\
\hline Type of data cube (resolution) & & high & low & high & low & high & low \\
\hline Applied weighting scheme & & uniform & robust 0.4 & uniform & robust 0.4 & uniform & robust 0.4 \\
\hline Pixel size (") & $\mathrm{d} x$ & 4 & 7 & 4 & 7 & 4 & 7 \\
\hline Channel width $\left(\mathrm{km} \mathrm{s}^{-1}\right)$ & $\mathrm{d} v$ & 2.06 & 4.12 & 2.06 & 4.12 & 2.06 & 4.12 \\
\hline$H P B W$ along the beam major axis $\left({ }^{\prime \prime}\right)$ & $H P B W_{\text {maj }}$ & 14.4 & 30.0 & 12.2 & 25.4 & 12.67 & 24.81 \\
\hline$H P B W$ along the beam minor axis ( $\left.{ }^{\prime \prime}\right)$ & $H P B W_{\min }$ & 12.0 & 25.2 & 11.4 & 27.1 & 11.4 & 21.51 \\
\hline Beam position angle $\left({ }^{\circ}\right)$ & $\mathrm{PA}_{\text {beam }}$ & 3.1 & 4.4 & -9.2 & -11.5 & -1.4 & -19.6 \\
\hline rms noise (mJy/beam) in the cubes & $\sigma_{\mathrm{rms}}$ & 0.81 & 0.45 & 0.84 & 0.49 & 0.59 & 0.39 \\
\hline rms noise $(\Delta v=2 \cdot \mathrm{d} v)\left(10^{19}\right.$ atoms $\left.\mathrm{cm}^{-2}\right)$ & $\sigma_{\mathrm{rms}}^{N}$ & 2.14 & 0.54 & 2.74 & 0.65 & 1.86 & 0.65 \\
\hline $\operatorname{rms}$ noise $(\Delta v=2 \cdot \mathrm{d} v)\left(M_{\odot} \mathrm{pc}^{-2}\right)$ & $\sigma_{\mathrm{rms}}^{\odot}$ & 0.172 & 0.043 & 0.219 & 0.052 & 0.149 & 0.053 \\
\hline Max. rms noise, total intensity $\left(\mathrm{Jy} /\right.$ beam $\left.\mathrm{km} \mathrm{s}^{-1}\right)$ & $\sigma_{\text {rms, tot }}$ & 0.022 & 0.018 & 0.022 & 0.020 & 0.014 & 0.0135 \\
\hline Max. rms noise, total intensity $\left(10^{19}\right.$ atoms $\left.\mathrm{cm}^{-2}\right)$ & $\sigma_{\text {rms tot }}^{N}$ & 14.4 & 2.56 & 17.3 & 3.17 & 10.8 & 2.78 \\
\hline Max. rms noise, total intensity $\left(M_{\odot} \mathrm{pc}^{-2}\right)$ & $\sigma_{\mathrm{rms}, \text { tot }}^{\odot}$ & 1.15 & 0.205 & 1.39 & 0.254 & 0.864 & 0.223 \\
\hline
\end{tabular}

where the orientational parameters begin to change substantially. Then, visually comparing the original data cubes with the artificial data cubes stemming from the models with an inner flat disk and with a radius-dependent orientation of the inner disk, it was decided whether to accept a model with an inclined inner disk. Except for the case of NGC 2541, the data were found to be consistent with an inner flat disk, while for NGC 2541 the spectra were slightly better represented allowing for a change of the inner disk orientation.

In the region of the flat inner disk, the finally adopted error bars of the orientational parameters for UGC 3580 and
NGC 5204 are the difference between the original fit results without enforcing a flat inner disk and the best-fit results with enforcement of a flat inner disk. For NGC 2541 the previously determined error bars were adopted. For the outer disks of all galaxies, the error bars from the first fits were taken over.

In order to refine the spatial resolution of the rotation curve and the surface brightness profile, the high-resolution data cubes were used. Up to a maximal radius, the spacing of the ring radii was refined and the ring parameters and their error bars were determined via linear interpolation. The number of point sources was increased to a value between $5.0 \times 10^{6}$ and $1.4 \times 10^{7}$ in order 


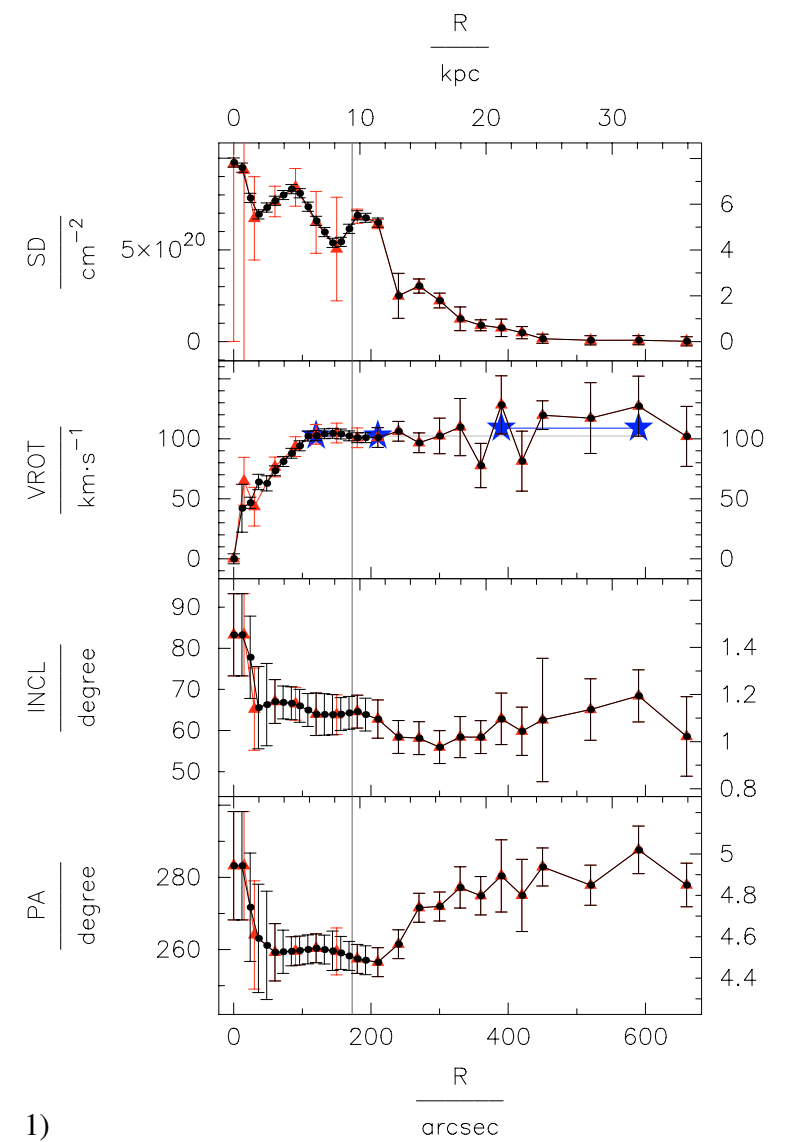

Fig. 1. Tilted-ring parametrisations of the galaxies (the figure is continued on subsequent pages). SD: surface density. VROT: rotation velocity. INCL: inclination. PA: position angle. R: radius. Dots and connecting lines (black in online version): finally adopted best-fit model. Triangles and connecting lines (red in online version): best-fit model fitting to the low-resolution data cube. Stars, connecting lines and grey line (blue and grey in online version): Best-fit velocities keeping the rotation curve flat for the regions enclosed by the stars. The vertical line denotes the optical radius $r_{25}$. NGC 2541 .

to then fit the rotation curve, the surface brightness profile, the global scaleheight, and the global velocity dispersion only in the selected region. For the final result, represented by the black dots in Fig. 1, the error bars in the surface brightness profile and the rotation velocity were again derived by fitting to the receding and approaching halves of the galaxy.

Several ways to check the quality of the finally resulting fits are possible and have been exhausted, as shown in detail in Józsa (2006). Figure 2 shows overlays of corresponding moment maps of the original data and the synthetic ones. As with TiRiFiC one fits to the unmasked data cube, it is possible to extend the model beyond the derived moment maps. Inside the radius where the total intensity and first moment maps were derived, a good agreement between data and model is found. For larger radii, PV diagrams (see Fig. 3) or the data cubes could be compared directly (see Fig. 5 in the online version of this paper). Table 3 shows the final adopted global parameters of the models. In all fits, the scaleheight with an assumed $\operatorname{sech}^{2}$-law and the dispersion were kept as free parameters. A rough estimate of the uncertainties of these quantities may be derived from the differences in the lowresolution fits and the high-resolution fits. The resulting values for both parameters lie in an acceptable range. As both values were fitted as global parameters they are not very meaningful in terms of a physical interpretation. It is, however, remarkable

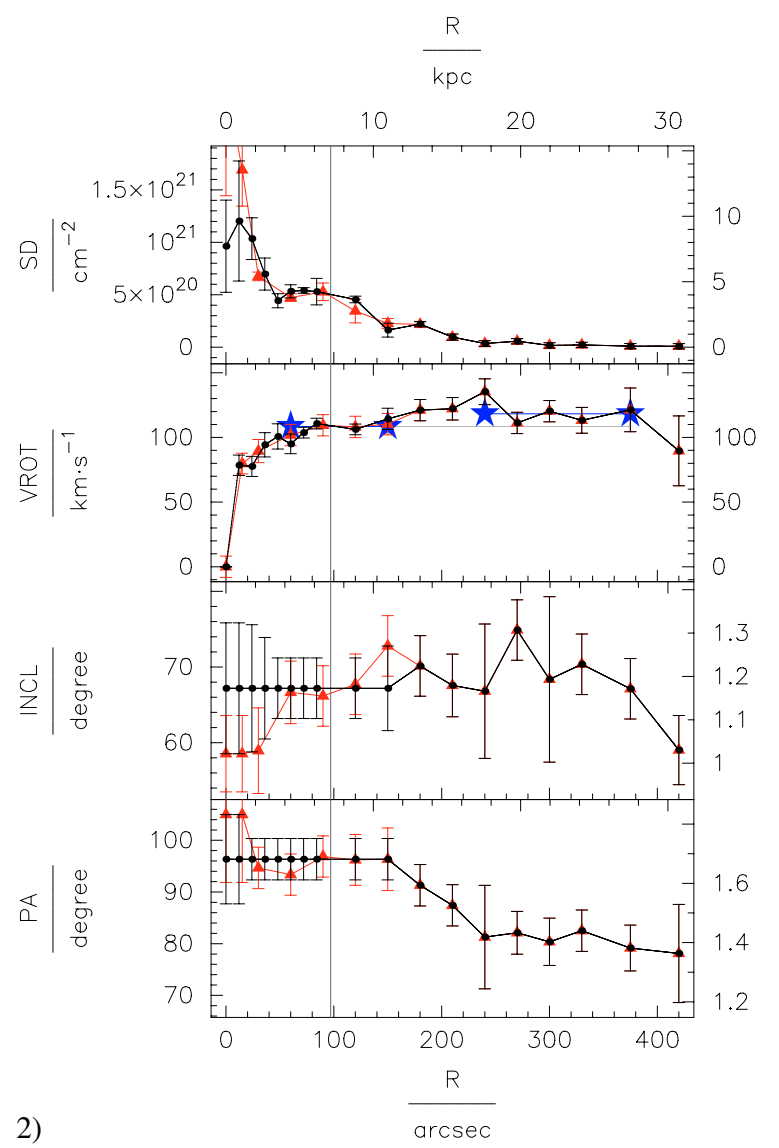

Fig. 1. continued. UGC 3580.

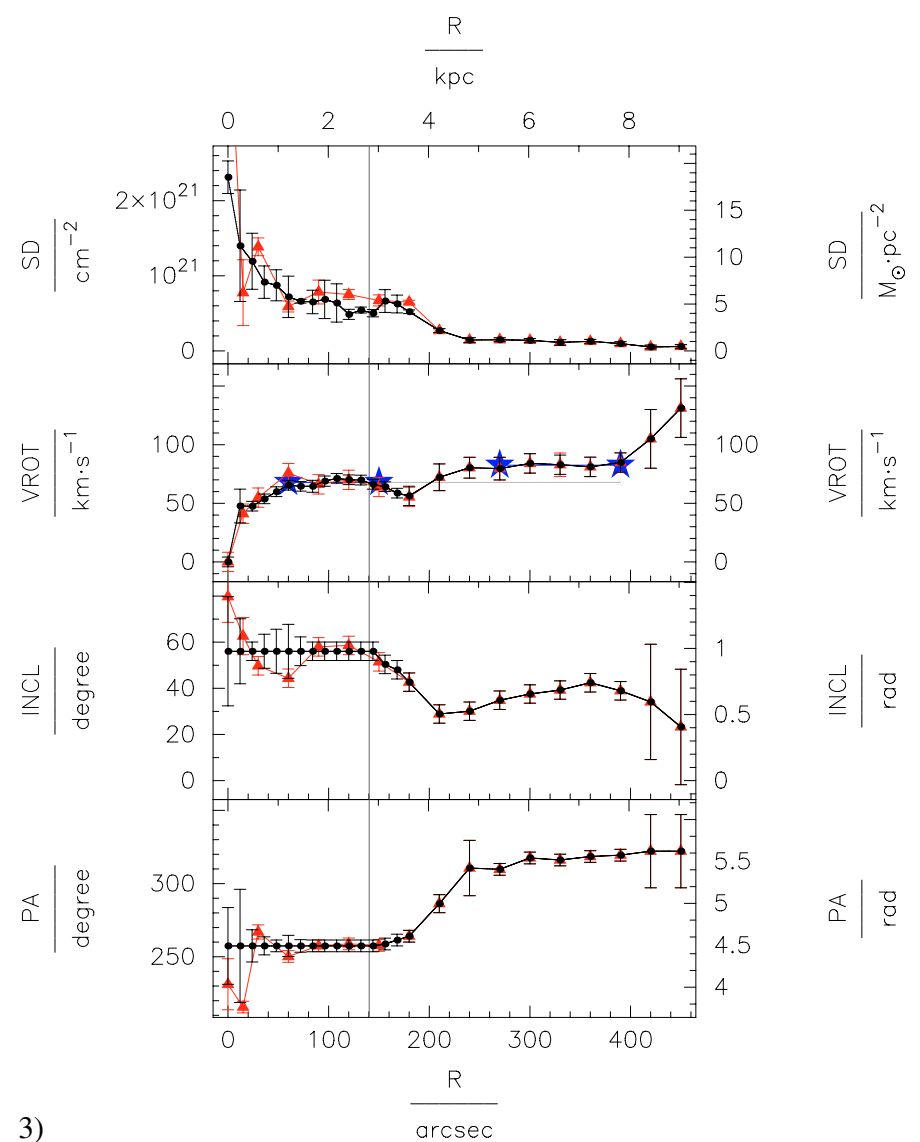

Fig. 1. continued. NGC 5204. 

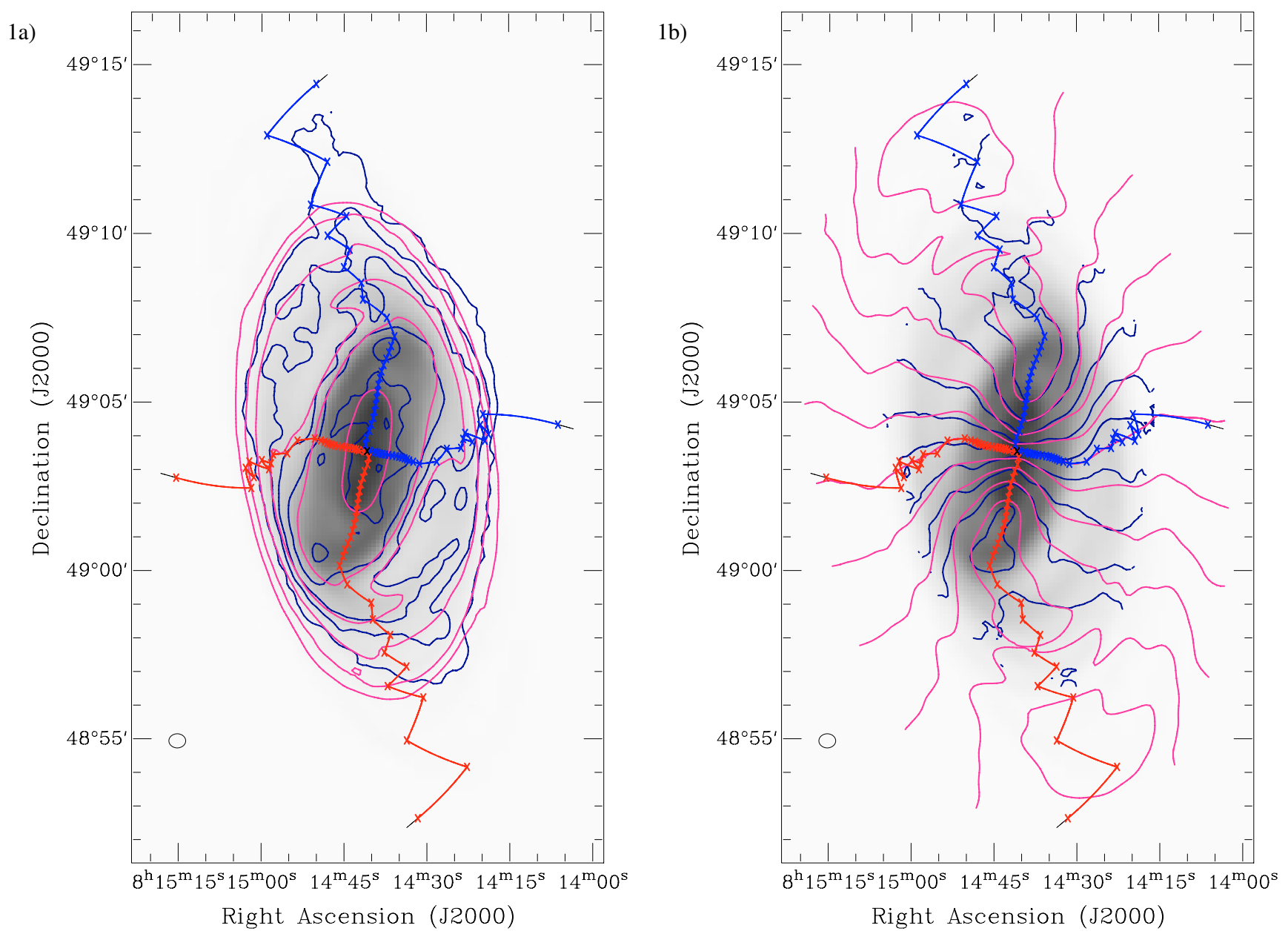

Fig. 2. Total intensity contour plots (a, left) and first moment velocity fields (b, right) derived from the low-resolution data cubes, blue original, pink model, overlaid on total intensity grey-scale maps derived from the model data cubes. The over-plotted lines represent the kinematical major and minor axes. The red kinematical major axis lies in the receding part of the galaxy, the blue kinematical major axis in the approaching part.

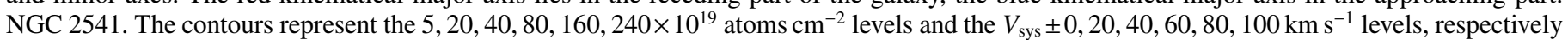
(see Table 1).

that the velocity dispersion found for the high-resolution data is systematically higher than found for the low-resolution data and that the scaleheight is systematically lower. A simple explanation lies in the weighting scheme applied to make both data cubes. For the high-resolution data, the detected $\mathrm{H}$ I is more confined to the disk as the fitted scaleheight is lower. This indicates that in the low-resolution data cubes, a smoother component is detectable that is not as confined to the disk.

Remarkably, a close look at the PV diagrams (Fig. 3) shows that, at the lowest contour levels, all target galaxies possess an excess in the width of the velocity profiles in the central regions. This is most evident for NGC 5204, but also visible in the case of NGC 2541 and UGC 3580, as a comparison with the model data cubes and PV diagrams shows. A single-disk tilted-ring model is not able to completely describe the gas distributions at the faintest level. Such features have been detected and analysed in other galaxies (e.g. Fraternali et al. 2004) and have been interpreted as a gas component that belongs to a thickened disk or a halo of neutral gas, which rotates at a lower rotation speed than the central disk. Hence, it is not surprising that the scale height resulting from the low-resolution fits is higher. This is because the halo gas, detected with a higher sensitivity in the lowresolution cubes, partly alters the results of the fits with respect to the scale height. However, since the effect is visible where the emission of the galaxy is strongest, the single component tiltedring models for the central disk presented here are not likely to be strongly affected by the presence of this excess component. The issue of the anomalous gas components present in all of the three sample galaxies is followed up (see Józsa et al. 2007b) and results will be reported elsewhere.

At the same time the fitted velocity dispersion is higher for the high-resolution data cubes. This can be explained by the fact that the fitted velocity dispersion is determined primarily by local deviations in the disk from the assumed symmetry. It fits into this picture that NGC 2541, which is the galaxy with the most pronounced spiral arms in the small sample, shows the most obvious deviations in the velocity dispersion comparing high-and low-resolution fits and, at the same time, the largest uncertainties in the parametrisation of the radially dependent parameters. Table 6 in the online version of this paper lists the radially dependent best-fit parameters shown in Fig. 1.

\section{Distance estimation and global parameters}

In order to estimate some of the global parameters shown in Table 1, a distance to the galaxies has to be assumed. In the 
2a)

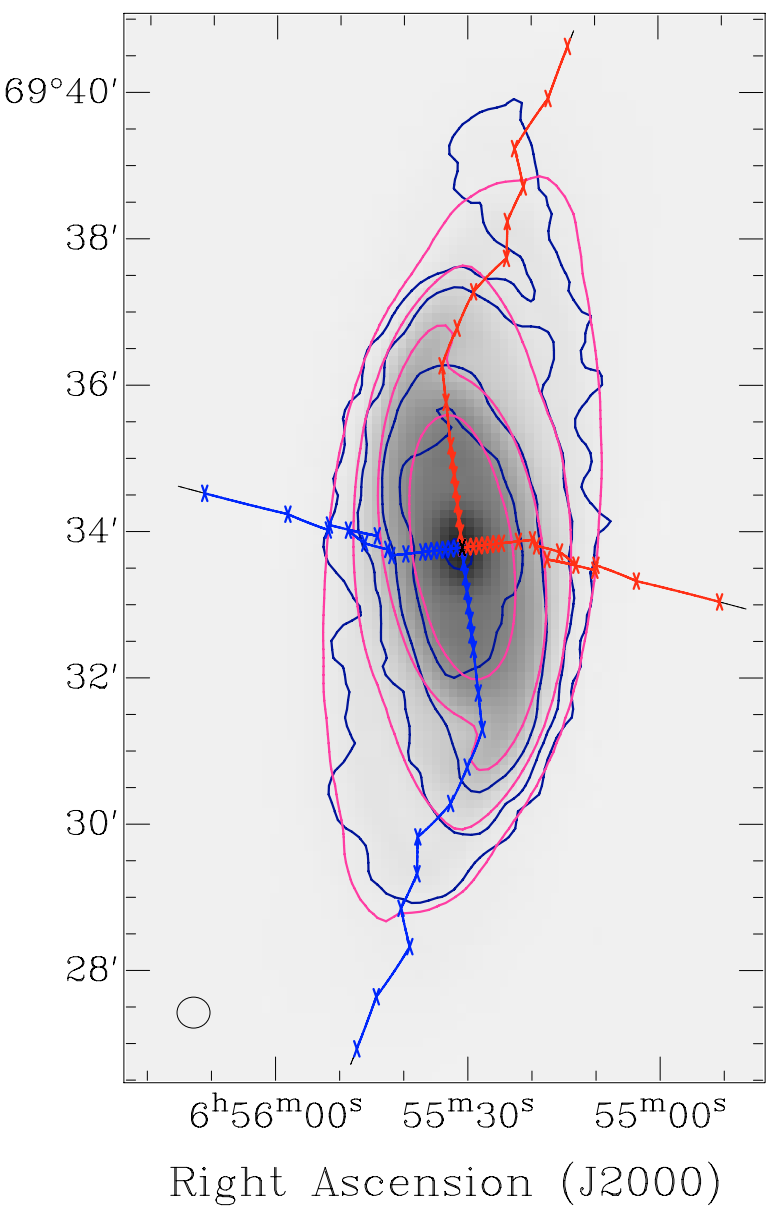

2b)

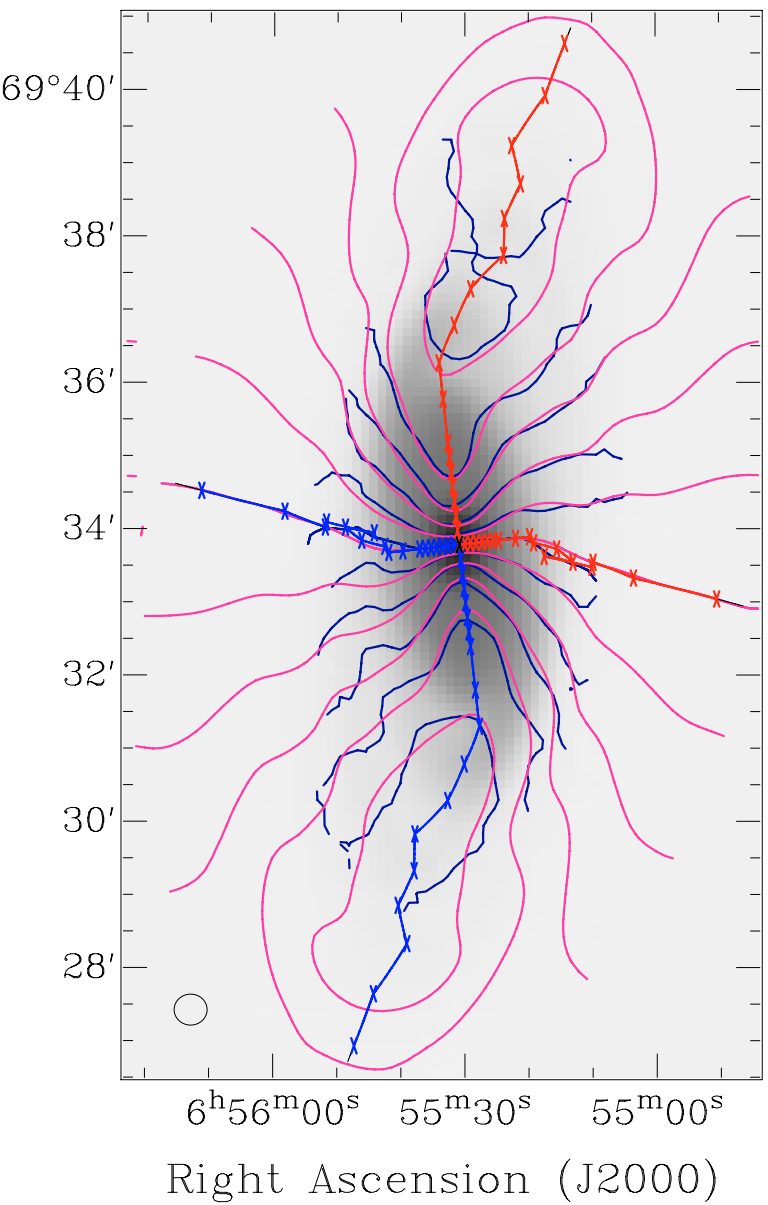

Fig. 2. continued. UGC 3580. The contours represent the $6,19,57,114,228 \times 10^{19}$ atoms cm ${ }^{-2}$ levels and the $V_{\text {sys }} \pm 0,20,40,60,80,100 \mathrm{~km} \mathrm{~s}^{-1}$ levels, respectively (see Table 1).

case of NGC 2541, the Cepheid distance given in Freedman et al. (2001) was adopted. For the two remaining galaxies, having $\mathrm{H}$ I data available, together with $I$-band magnitudes from the literature, the Tully-Fisher relation as described in Sakai et al. (2000) was applied (for details see Józsa 2006). For the necessary estimation of the HI linewidth, the derived models were used. A comparison for NGC 2541 of the Tully-Fisher distance of (11.6 \pm 2.6$) \mathrm{Mpc}$ with the cepheid distance of $(11.22 \pm 0.8) \mathrm{Mpc}$ as derived by Freedman et al. (2001) shows that this method works well. In order to estimate a lower limit to the dynamical masses of the galaxies, the terminal radius $R_{\mathrm{t}}$ (in physical units) was determined as the second-largest radius in the best-fit models and the dynamical mass

$M_{\text {dyn }}=\frac{R_{\mathrm{t}} V_{\mathrm{t}}^{2}}{G}$

inside that radius was calculated with the best-fit rotation velocity $V_{\mathrm{t}}$ at that radius and the gravitational constant $G$.

The scales for the luminosities were defined by $M_{\odot}^{\mathrm{B}}=5$. 48 in the $B$-band and $M_{\odot}^{\mathrm{I}}=4^{\mathrm{m}} .08$ in the $I$-band (cf. Binney \& Merrifield 1998).

\section{Global features in the tilted-ring models}

The finally adopted tilted-ring models (Fig. 1) can be visualised in different ways, as shown in Fig. 4 (see also Figs. 9 and 10 in the online version). Up to a certain radius, basically all rules for the behaviour of warps formulated by Briggs (1990) (rules i-iv in Sect. 1) are followed by the three galaxies within the given error bars. The disks stay flat out to approximately $r_{25}$ and start to show the warp at somewhat larger radii. Panels a) and b) in Fig. 4 show the tip-LON plots introduced by Briggs (1990) in order to develop his rules. They show the projection of the normal vector of each ring onto a plane, the distance to the origin indicating the so-called warp angle or tip angle, the azimuthal angle being the so-called line-of-the-nodes (LON). Briggs' first, second, and third rules can be checked by inspecting tip-LON plots in which the reference plane is chosen to be the central (flat) plane of the galaxies as shown in Fig. 4b. Linear plots containing the errors of the warp (tip-) angle and the LON are presented in the online version of this paper (Fig. 10, see also Table 6). According to the first rule, the line of the nodes should stay straight for a few data points in the region of the commencement of the warps; in other words, the tips of the normal vectors should lie on one straight line that goes through the origin of the plots. While this is barely visible in the case of UGC 3580, which has the warp with the smallest amplitude in the sample, this feature is apparent for NGC 2541 and NGC 5204. Beyond a certain radius $r_{\text {tr }}$ according to Briggs' rules, the LONs should form a leading spiral. Optical images of the galaxies were inspected in order to determine the sense of rotation from dust-lane features or from the orientation of spiral arms, assumed to be trailing. In Fig. 4a) the sense of rotation is indicated by an arrow. The plots (compare 

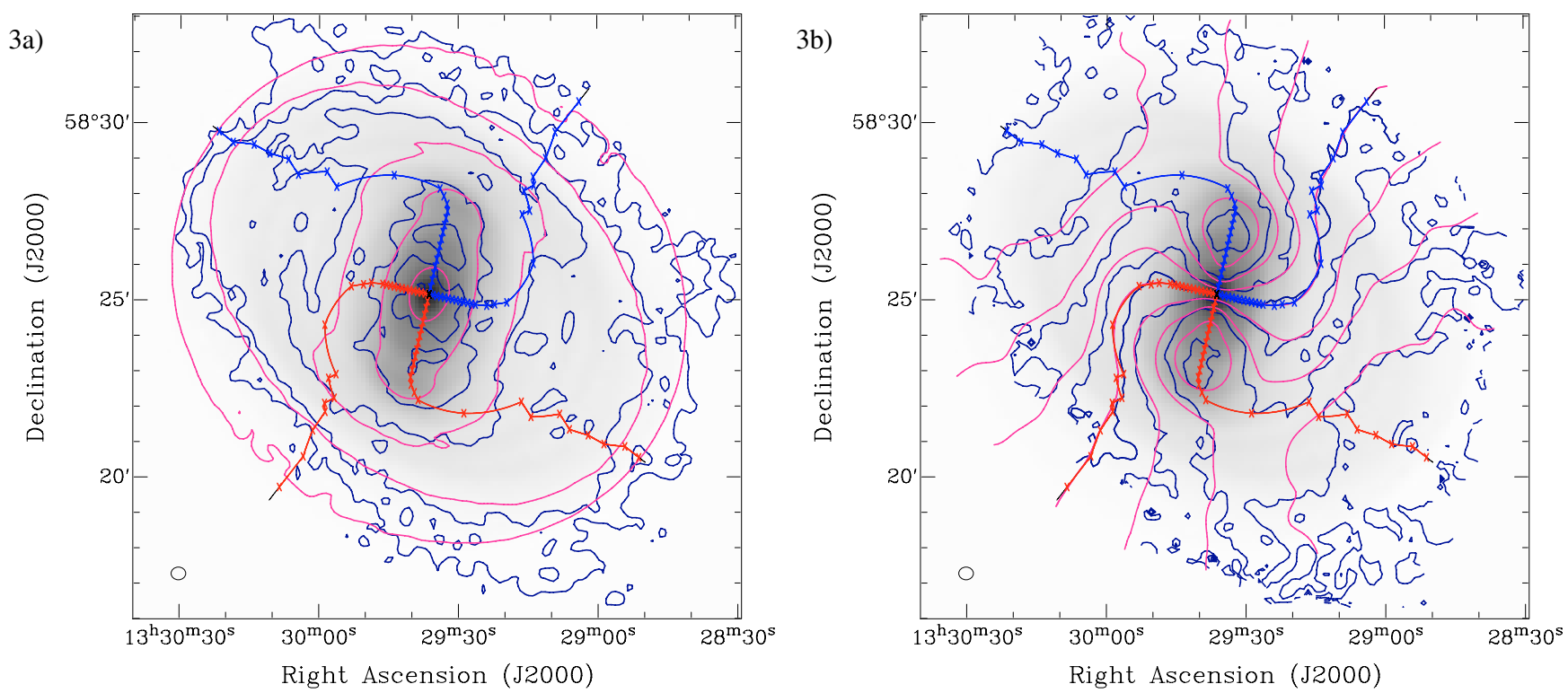

Fig. 2. continued. NGC 5204. The contours represent the $2.5,8,25,75,145,225 \times 10^{19}$ atoms cm ${ }^{-2}$ levels and the $V_{\text {sys }} \pm 0,15,30,45,60 \mathrm{~km} \mathrm{~s}^{-1}$ levels, respectively (see Table 1).
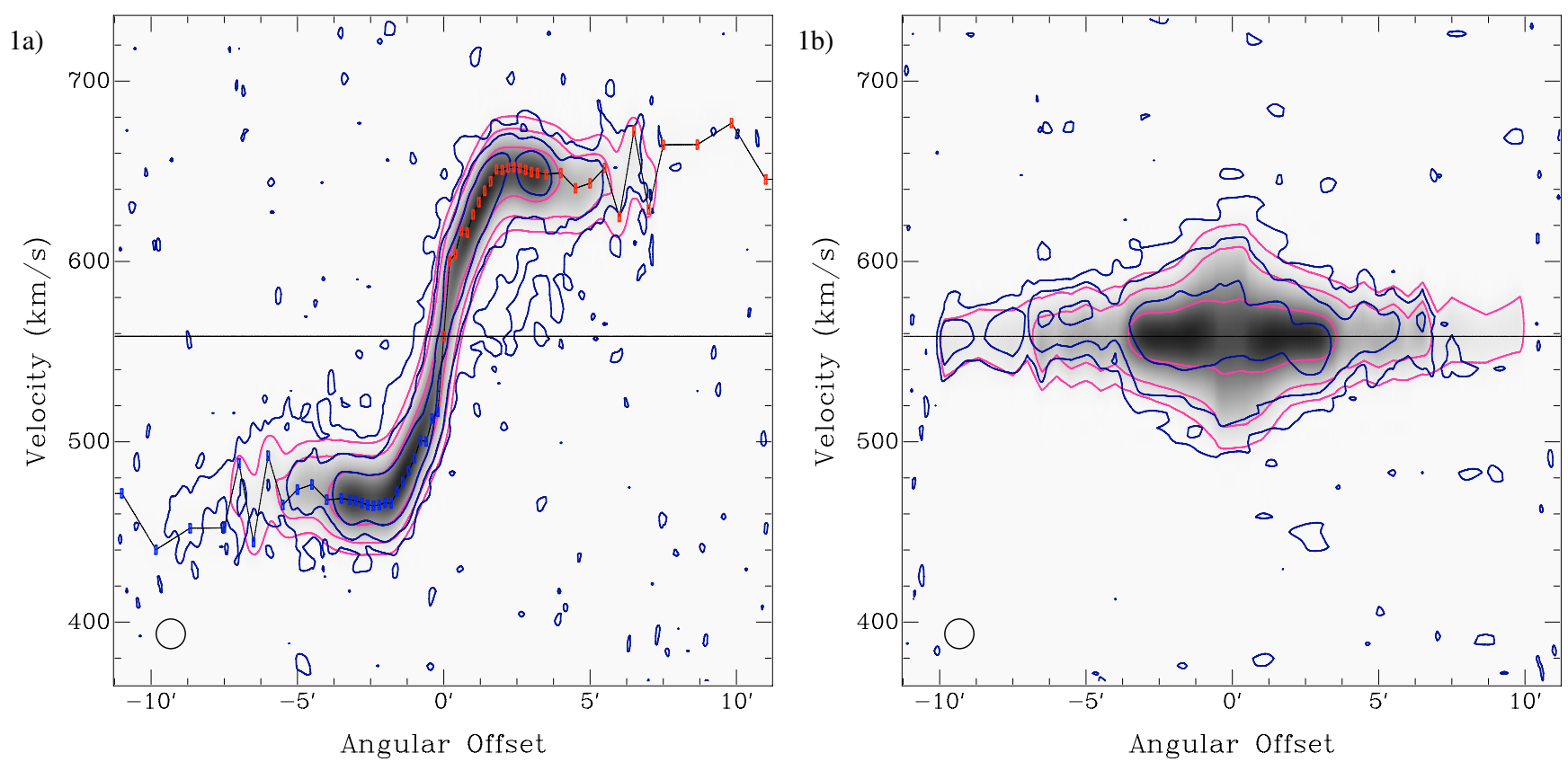

Fig. 3. a) PV diagrams along the kinematical major axis comparing the final best-fit models ( light grey contours, pink in online version) with the original low-resolution data ( dark grey contours, blue in online version). The vertical line marks the systemic velocity, the boxes connected with the lines denote the rotation curve corrected for the inclination. b) PV diagrams along the kinematical minor axis comparing the final models ( light grey contours, pink in online version) with the original low-resolution data (dark grey contours, blue in online version). The ellipses in the lower left corner indicate the major axis $H P B W$ and the velocity resolution (FWHM). NGC 2541. Contour levels are 1, 4, 16 mJy/beam. NGC 2541 .

with Fig. 10 in the online version) show that within the errors, rule iii) is followed up to a certain radius. For larger radii the data points do not show a clear tendency toward a spirality, but the LON stays comparably constant.

Also the question whether the "forgotten" fourth rule of Briggs is obeyed by the sample galaxies can be answered. His observation that a reference frame can be found in which two straight lines of the nodes exist, one for the inner part of the galaxy and one for the outer part, has been a matter of discussion and has since been rejected by Briggs himself
(Briggs \& Kamphuis 1991). However, this rule is reproducible with the parametrisations derived here. Figure $4 \mathrm{c}$ shows the attempts to find the reference plane in which this feature is most visible.

Figure 4c shows "tiltograms" of the galaxy parametrisations, i.e. images in which the inclination $\theta_{\text {tilt }}$ of a (sub-)ring with a (sub-)ring at a different radius is colour-coded (see also Fig. 11 in the online version of the paper). Light (yellow) areas in the diagram show regions where the mutual inclination is low, and dark (blue) areas show regions where the mutual inclination is 


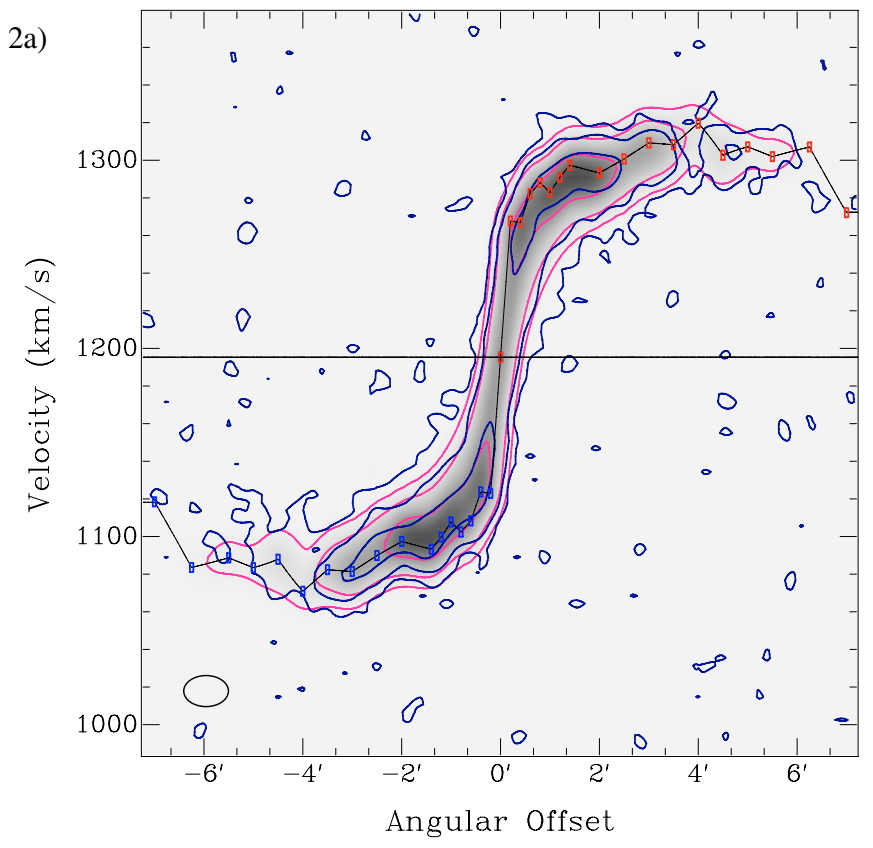

Fig. 3. continued. UGC 3580. Contour levels are 1, 4, $16 \mathrm{mJy} /$ beam.

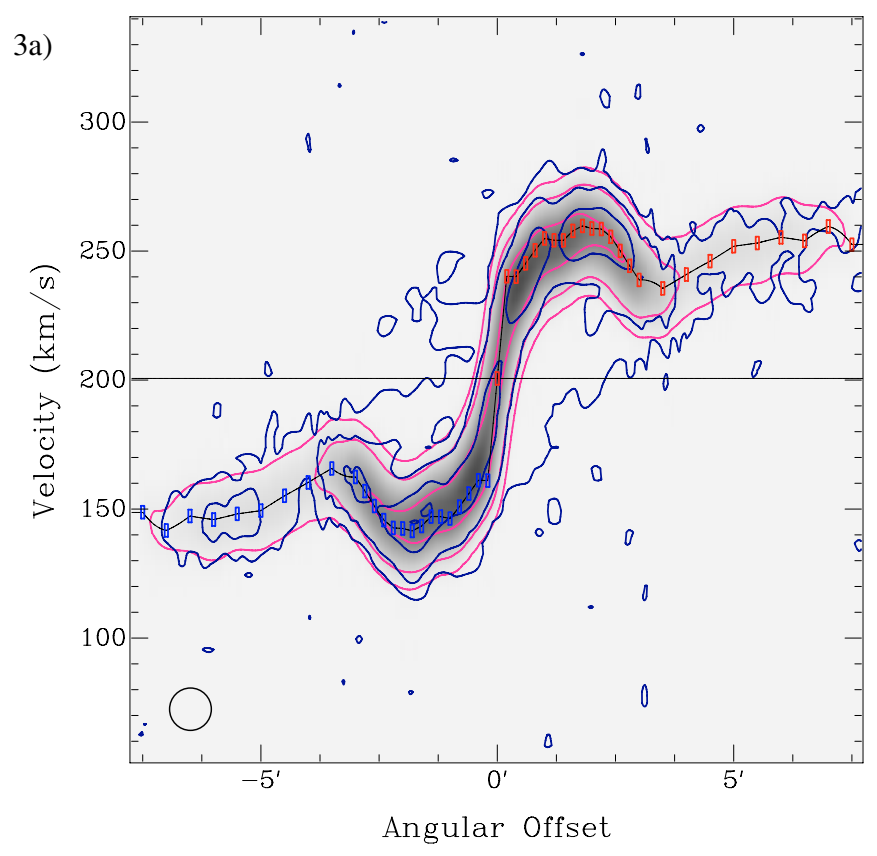

Fig. 3. continued. NGC 5204. Contour levels are 1, 4, 16, 32 mJy/beam.

high. These tiltograms are a representation of the orientational parameters of the tilted-ring model that is independent of the orientation of the galaxy with respect to the observer. The existence of two well-separated, roughly rectangular regions in the diagrams within which the pixel values do not change much, indicates that all three H I disks show two separate regions within which the orientational parameters do not change significantly and that these two regions are misaligned with each other. The same diagram for a galaxy with a disk that continuously changes orientation towards larger radii would not show two rectangular regions; it would rather exhibit one rectangular region
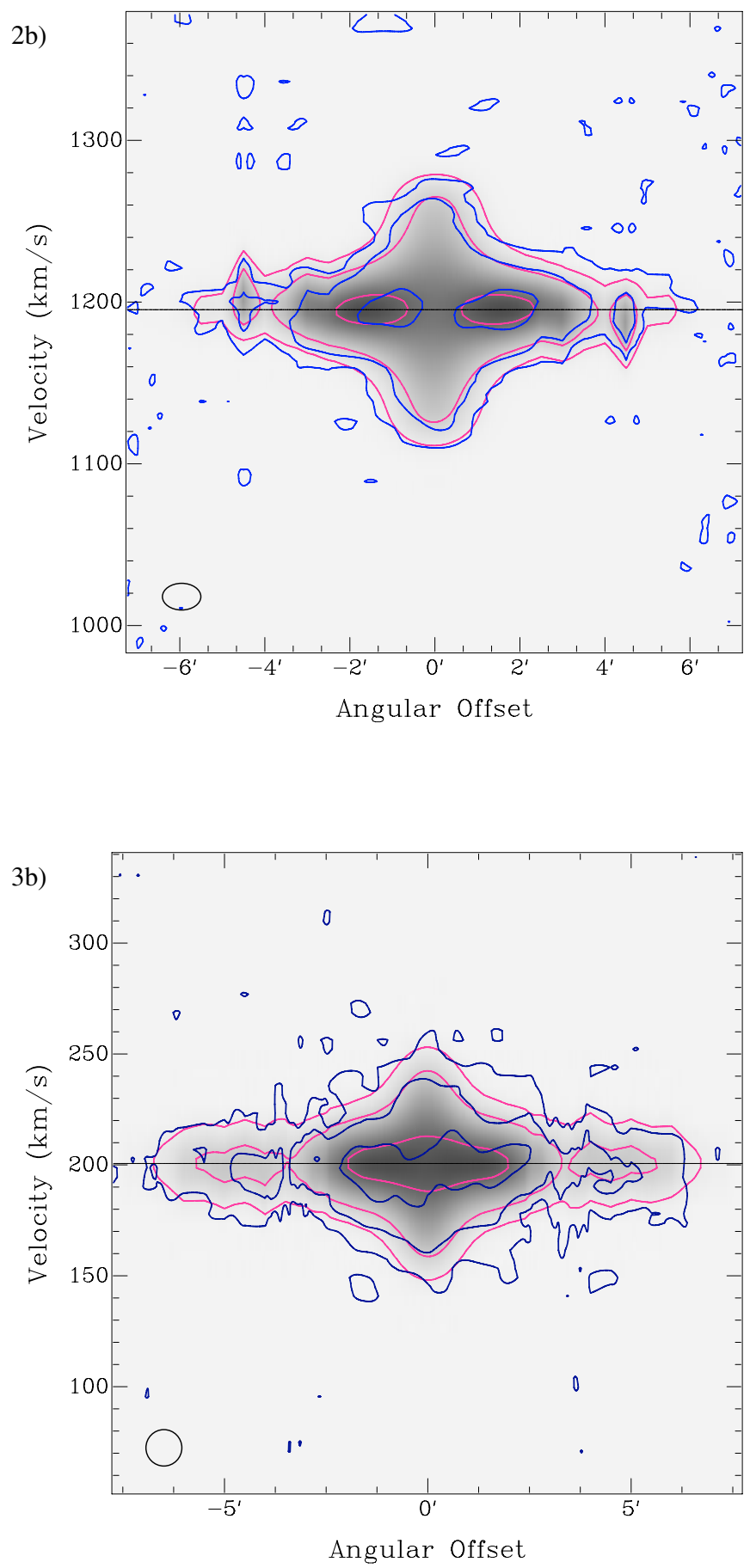

representing a flat inner disk and a tail of lower values in the vicinity of the diagonal. The galaxies seem to show outer and an inner flat disks that are connected by a region in which the disk orientation changes more or less rapidly. For a more quantitative approach, the best-fit parameters from the fit to the lowresolution data cubes without enforcing an inner flat disk (Fig. 1, see also Fig. 11 in the online version) were used to identify two ranges of radii, an inner one and an outer one, within which the orientational parameters stay comparably constant (listed in Table 4). Then, the mean and the standard deviation of the intrinsic mutual inclination of the rings within these regions, $\bar{\theta}_{\text {tilt }}^{\text {in }}$ 
Table 3. Global best-fit tilted-ring parameters for the examined galaxies.

\begin{tabular}{|c|c|c|c|c|c|c|c|}
\hline & \multicolumn{2}{|c|}{ NGC 2541} & \multicolumn{2}{|c|}{ UGC 3580} & \multicolumn{2}{|c|}{ NGC 5204} \\
\hline Right Ascension (J2000), fitting results & $\mathrm{RA}_{\text {fit }}$ & \multirow{2}{*}{\multicolumn{2}{|c|}{$\begin{array}{l}08^{\mathrm{h}} 14^{\mathrm{m}} 40^{\mathrm{s}} .83 \\
+49^{\circ} 03^{\prime} 33^{\prime \prime} 6\end{array}$}} & \multicolumn{2}{|c|}{$06^{\mathrm{h}} 55^{\mathrm{m}} 30^{\mathrm{s}} .89$} & \multicolumn{2}{|c|}{$13^{\mathrm{h}} 29^{\mathrm{m}} 36^{\mathrm{s}} .30$} \\
\hline Declination (J2000), fitting results & $\operatorname{Dec}_{\text {fit }}$ & & & \multirow{2}{*}{\multicolumn{2}{|c|}{$\begin{array}{l}+69^{+} 33^{2} 47.4 \\
06^{\mathrm{h}} 55^{\mathrm{m}} 30.86\end{array}$}} & \multicolumn{2}{|c|}{$+58^{\circ} 25^{\prime} 10^{\prime \prime} .8$} \\
\hline Right Ascension (J2000) from NED & $\mathrm{RA}_{\mathrm{NED}}$ & \multicolumn{2}{|c|}{$08^{\mathrm{h}} 14^{\mathrm{m}} 40^{\mathrm{s}} 07$} & & & $13^{\mathrm{h}} 2$ & 36.51 \\
\hline Declination (J2000) from NED & $\operatorname{Dec}_{\mathrm{NED}}$ & \multicolumn{2}{|c|}{$+49^{\circ} 03^{\prime} 41^{\prime \prime} \cdot 2$} & \multicolumn{2}{|c|}{$+69^{\circ} 33^{\prime} 47^{\prime \prime} 0$} & \multicolumn{2}{|c|}{$+58^{\circ} 25^{\prime} 07^{\prime \prime} .4$} \\
\hline Heliocentric optical systemic velocity $\left(\mathrm{km} \mathrm{s}^{-1}\right)$ & $V_{\text {sys }}$ & \multicolumn{2}{|c|}{559.5} & \multicolumn{2}{|c|}{1200.1} & \multicolumn{2}{|c|}{200.8} \\
\hline Type of data cube used for analysis, high-resolution or low-resolution & & high & low & high & low & high & low \\
\hline Dispersion $\left(\mathrm{km} \mathrm{s}^{-1}\right)$ & $V_{\text {disp,tot }}$ & 13.4 & 11.4 & 9.6 & 9.4 & 9.4 & 9.7 \\
\hline Internal dispersion, instrumental dispersion subtracted $\left(\mathrm{km} \mathrm{s}^{-1}\right)$ & $V_{\text {disp,int }}$ & 13.3 & 10.8 & 9.4 & 8.7 & 9.2 & 9.0 \\
\hline Global scaleheight (") & $z_{0}$ & 11.5 & 12.1 & 7.4 & 9.7 & 15.5 & 20.6 \\
\hline Global scaleheight (pc) & $z_{0}^{\mathrm{pc}}$ & 628 & 656 & 545 & 708 & 312 & 414 \\
\hline
\end{tabular}
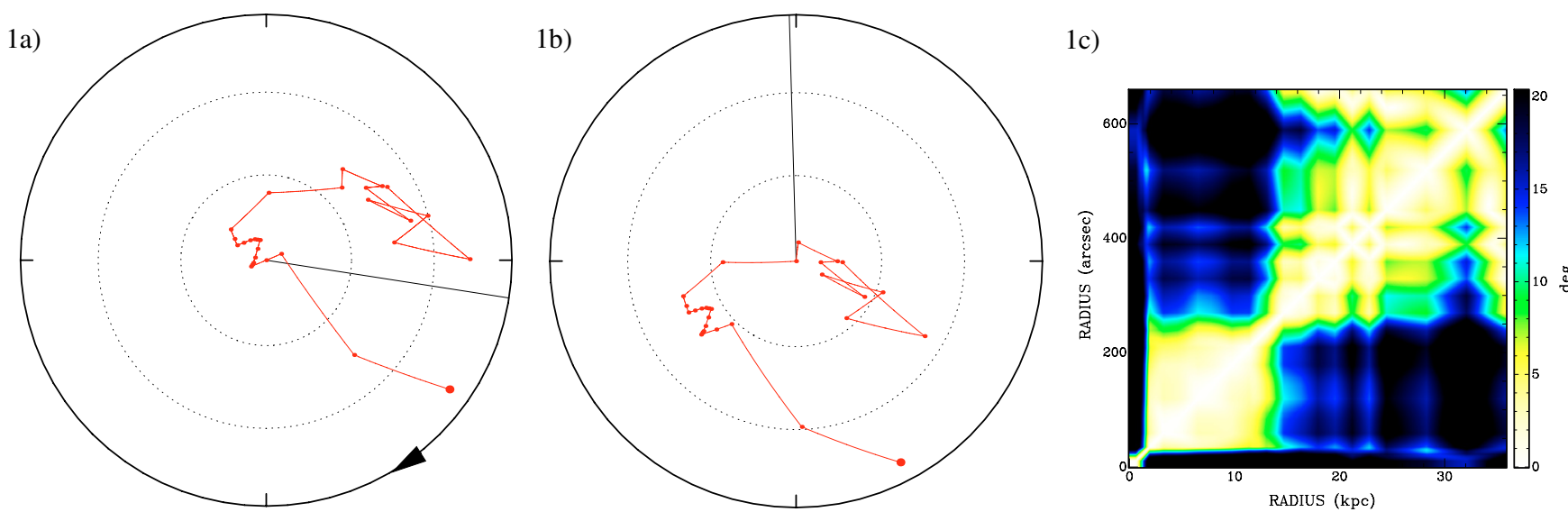

Fig. 4. Various visualisations of the adopted tilted-ring models for the galaxies. a) and b) tip-LON plots, the filled circles are the projection of the tip of the normal vectors of the rings at different radii onto a reference plane the innermost radius (0) denoted by a larger circle. The circles in panels a) and b) are drawn at intervals of $10^{\circ}$. a) The reference plane is determined by the orientation of the fifth ring (see Fig. 1 and Table 6 in the online version). The arrows indicate the sense of rotation. b) The reference plane is chosen such that two straight LONs are visible, resembling the fourth rule of Briggs (1990). At larger radii the dots are clustered around the same position, indicating an outer planar disk. c) Tiltogram of the galaxies. This is a pixel map showing the mutual inclination $\theta_{\text {tilt }}$ of rings at different radii. Most clearly, this diagram shows the existence of two planar disks, an inner one and an outer one (see also Fig. 11 for a comparison). Note that large errors are assigned to the last data points at the largest radii (see Fig. 10 in the online version). NGC 2541. The first two data points in a) and b) are indicative of an inner tilt of the disk of this galaxy or an erroneous fit due to non-circular motions in the innermost region of this galaxy.
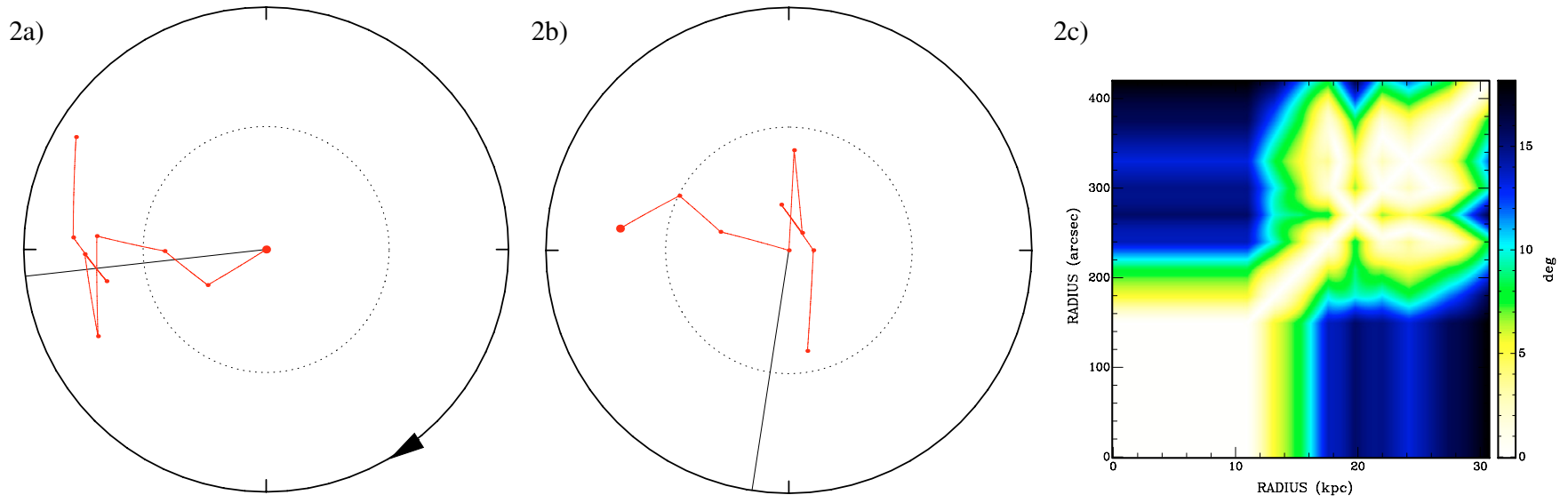

Fig. 4. continued. UGC 3580.

and $\bar{\theta}_{\text {tilt }}^{\text {out }}$, and the mean of the mutual inclination taking into account only ring-pairs consisting of one ring from the inner disk and one ring of the outer disk $\bar{\theta}_{\text {tilt }}^{\text {mut }}$ was determined (the corresponding regions in the tiltograms are marked in Fig. 11 in the online version of this paper). In order to get a reference for an intrinsic scatter representative of a flat disk, the best-fit parameters fitting to the low-resolution data cube without enforcing an inner flat disk were used. As shown in Table 4, the differences $\bar{\theta}_{\text {tilt }}^{\text {in }}-\bar{\theta}_{\text {tilt }}^{\text {out }}$ are close to zero, while $\bar{\theta}_{\text {tilt }}^{\text {mut }}-\bar{\theta}_{\text {tilt }}^{\text {in }}$ and $\bar{\theta}_{\text {tilt }}^{\text {mut }}-\bar{\theta}_{\text {tilt }}^{\text {out }}$ differ significantly from zero. The flatness of the outer disk $\bar{\theta}_{\text {tilt }}^{\text {out }}$ is comparable to that of the inner disk $\bar{\theta}_{\text {tilt }}^{\text {in }}$, while the change in the 

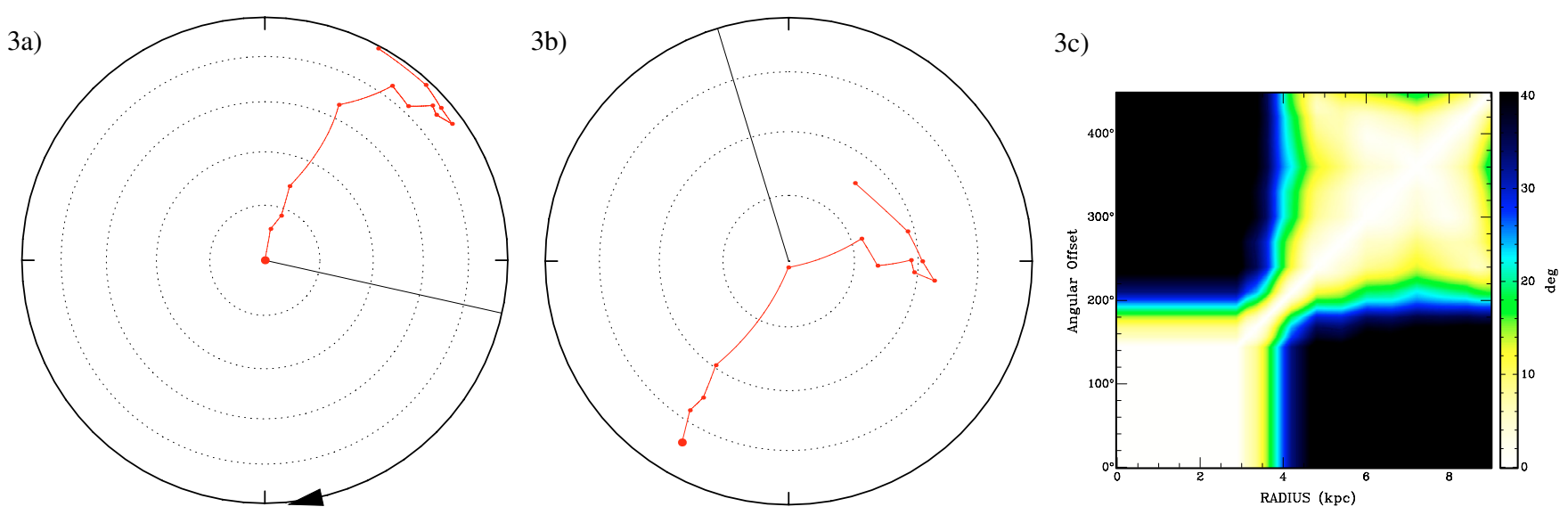

Fig. 4. continued. NGC 5204.

Table 4. Mean mutual inclinations of the galaxy disks, comparing different regions (see Sect. 6 and Fig. 11 in the online version).

\begin{tabular}{|c|c|c|c|}
\hline Galaxy name & NGC 2541 & UGC 3580 & NGC 5204 \\
\hline Range of inner disk (") & $30-240$ & $30-180$ & $30-180$ \\
\hline Range of inner disk (kpc) & $300-590$ & $240-375$ & $240-420$ \\
\hline Range of outer disk ( $\left.{ }^{\prime \prime}\right)$ & $1.6-13.1$ & $2.2-13.2$ & $0.6-3.6$ \\
\hline Range of outer disk (kpc) & $16.3-32.1$ & $17.6-27.5$ & $4.8-8.4$ \\
\hline Intrinsic mean mutual inclination of inner disk $\bar{\theta}_{\text {tilt }}^{\text {in }}\left({ }^{\circ}\right)$ & $3.4 \pm 2.6$ & $4.6 \pm 4.0$ & $7.5 \pm 5.9$ \\
\hline Intrinsic mean mutual inclination of outer $\operatorname{disk} \bar{\theta}_{\text {tilt }}^{\text {out }}\left({ }^{\circ}\right)$ & $5.8 \pm 4.9$ & $2.9 \pm 2.9$ & $6.2 \pm 2.9$ \\
\hline Mean mutual inclination of inner and outer disk $\theta_{\text {tilt }}^{\text {mut }}\left({ }^{\circ}\right)$ & $17.6 \pm 4.4$ & $13.9 \pm 2.5$ & $41.1 \pm 5.7$ \\
\hline $\bar{\theta}_{\mathrm{tilt}}^{\text {mut }}-\bar{\theta}_{\mathrm{tilt}}^{\text {in }}\left({ }^{\circ}\right)$ & $14.3 \pm 5.1$ & $9.3 \pm 4.7$ & $33.7 \pm 8.2$ \\
\hline $\bar{\theta}^{\text {tilt }}-\bar{\theta}^{\text {tilt }}\left({ }^{\circ}\right)$ & $11.8 \pm 6.5$ & $10.9 \pm 3.8$ & $34.9 \pm 6.4$ \\
\hline $\bar{\theta}_{\text {tilt }}^{\text {tilt }}-\bar{\theta}_{\text {titl }}^{\text {oult }}\left({ }^{\circ}\right)$ & $-2.4 \pm 5.5$ & $1.7 \pm 4.9$ & $1.3 \pm 6.6$ \\
\hline
\end{tabular}

orientation $\bar{\theta}_{\text {tilt }}^{\text {mut }}$ is significant compared to the intrinsic flatness $\bar{\theta}_{\text {tilt }}^{\text {out }}$ or $\bar{\theta}_{\text {tilt }}^{\text {in }}$.

The most figurative visualisation of this feature is given by a three-dimensional model of the galaxies shown in Fig. 9 in the online version of this paper. These two disks can be readily seen in all visualisations shown here. At large radii, the orientational parameters remain constant within the error bars in Fig. 1, and the data points that belong to large radii cluster about the same position in the tip-LON plots. Knowing this, it is a simple task to find a realisation of a tip-LON plot in which the two "straight" lines of the nodes are visible. The fundamental plane of the plot has to be chosen to be the plane in which a ring lies that is in the intermediate region between the two disks. This position can be determined by looking at the tiltogram as the radius at which the two regions make contact. It is then not surprising that rule 4 is obeyed by the sample galaxies, as a few rings lie in the intermediate region that must connect two clusters of points in the tipLON diagram. If the transitional region where the disk changes from one orientation to the other is small, two "straight" lines of the nodes are seen looking at a tip-LON plot.

A further question is whether the changes in the disk orientation are accompanied by other signs of a change in the physical conditions for the disk at the transition radius, where the warp commences. An answer can be found by inspecting Fig. 1. At about the transition radius both the rotation curve and the surface brightness profiles change their behaviour with varying significance.

The surface density shows a drop and reaches a regime in the region of the outer disk where the gradient of the profile becomes very shallow, a feature that has already been connected with the warping phenomenon by García-Ruiz et al. (2002). This drop is well-defined in the case of NGC 2541 and NGC 5204, but is less significant for UGC 3580 . In Table 1 the H I radius $r_{\mathrm{HI}_{\mathrm{I}}}$ is included as the radius at which this transition occurs.

The rotation velocity of NGC 5204 shows a distinct feature: the rotation curve rises at the transition radius and then becomes comparably flat again in the region of the outer disk. While this feature is not easily seen in the viewgraphs for the two other cases (Fig. 1), an attempt was made to check whether there are indications of its existence and to possibly quantify it in all cases.

In order to have a robust estimate of the amplitude of a possible change in rotation velocity at the warp radius, another series of fits to the low-resolution data was performed. For each galaxy, two regions were selected for which the rotation curve was relatively flat in the best-fit models, one inner region and one outer region. Then, for both regions, as a first guess, the same average rotation velocity was assigned. A fit was performed for each galaxy, leaving all parameters variable as in the initial fitting process (also the orientational parameters), keeping the rotation curve flat and at the same value in each separate region, and allowing these two rotation velocities to vary independently (see Józsa 2006 for details). Here, the resulting best-fit model is called "rise model". The change in the rotation velocity (1) derived from these models is listed in Table 5.

In order to estimate the significance of the change in the rotational velocity, a new test series of fits was performed identical to the latter with the only difference being that the two selected regions were forced to have the same rotational velocity. The resulting best-fit model from this fitting run is called the "flat model". Both data cubes were then compared by eye with the original ones. Since the differences show up at lower contour levels, it is not immediately evident in the data cubes whether a 
Table 5. Statistical evidence for a rise in the rotation curves from the inner flat regime to the outer flat regime. In order to test whether a rise in the rotation curve is present and statistically relevant, two fits per galaxy to the low-resolution data cube were performed. For each fit and each galaxy an inner and an outer region was selected within which the rotation curve was kept flat (see also Fig. 1). A first fit was performed allowing the regions in which the rotation velocity was kept flat to have a different velocity ("rise model"). A second fit was performed enforcing the two regions to have the same rotation velocity ("flat model"). All other parameters were left to vary freely as described in the text. The $\chi^{2}$ was calculated in two ways, once taking into account all data points in the data cube, and once taking into account only those pixels which significantly differed from zero in either model data cube. The ratios of the resulting $\chi^{2}$ probability densities support a rise in the rotation velocities outwards.

\begin{tabular}{|c|c|c|c|}
\hline Name of galaxy. & NGC 2541 & 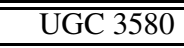 & NGC 5204 \\
\hline best-fit $\chi^{2}$ for flat model, calculated using the whole data cube. & $6.590 \times 10^{6}$ & $1.3999 \times 10^{6}$ & $4.605 \times 10^{6}$ \\
\hline best-fit $\chi^{2}$ for rise model, calculated using the whole data cube. & $6.565 \times 10^{6}$ & $1.3904 \times 10^{6}$ & $4.529 \times 10^{6}$ \\
\hline number of data points in the whole data cube. & $2.33 \times 10^{6}$ & $1.08 \times 10^{6}$ & $2.91 \times 10^{6}$ \\
\hline $\begin{array}{l}\text { probability density function } p\left(\chi^{2}\right) \text { of flat model divided by } p\left(\chi^{2}\right) \text { of rise model, } \\
\text { calculated for the whole data cube. }\end{array}$ & $1.3 \times 10^{-10}$ & $1.0 \times 10^{-2}$ & $2.3 \times 10^{-29}$ \\
\hline best-fit $\chi^{2}$ for flat model, derived only in regions with emission in the model data cubes. & $4.786 \times 10^{6}$ & $5.36 \times 10^{5}$ & $2.17 \times 10^{6}$ \\
\hline best-fit $\chi^{2}$ for rise model, derived in regions with emission in the model data cubes. & $4.760 \times 10^{6}$ & $5.26 \times 10^{5}$ & $2.09 \times 10^{6}$ \\
\hline number of data points in regions with emission in the model data cubes. & $5.35 \times 10^{5}$ & $2.02 \times 10^{5}$ & $2.91 \times 10^{6}$ \\
\hline $\begin{array}{l}\text { probability density function } p\left(\chi^{2}\right) \text { of flat model divided by } p\left(\chi^{2}\right) \text { of rise model, } \\
\text { calculated only in regions with emission in the model data cubes. }\end{array}$ & $2.6 \times 10^{-16}$ & $1.3 \times 10^{-5}$ & $8.0 \times 10^{-73}$ \\
\hline rotation velocity of the inner disk in the rise model $\left(\mathrm{km} \mathrm{s}^{-1}\right)$. & 102.1 & 108.9 & 67.9 \\
\hline rise model $\left(\mathrm{km} \mathrm{s}^{-1}\right)$. & 6.7 & 9.7 & 14.6 \\
\hline change of rotation velocity from inner to outer disks in the rise model. & $6 \%$ & $9 \%$ & $22 \%$ \\
\hline
\end{tabular}

step in velocity is necessary to reproduce the original data cubes. But a close inspection shows that the original data cube is represented better for NGC 5204 and UGC 3580 when allowing for a change in rotation velocity, while for NGC 2541 the quality of the fit when allowing for an independent variation of the rotation velocity in both regions was marginally better and did not allow an ad hoc conclusion. As an illustration Fig. 12 in the online version of the paper shows comparisons of PV diagrams derived from both (rise- and flat) model data cubes with PV diagrams derived from the original data. A purely statistical treatment, however, favours in all three cases the existence of a rise in the rotation velocity from inner to outer disks (see Table 5). In all three cases, $\chi^{2}$ was lower for the best-fit models allowing for two regions with differing velocities. The ratio of the $\chi^{2}$ probability functions was calculated with the number of data points in the data cubes. The volume of the $3 \mathrm{D}$ instrumentational function can be calculated from Table 2 and was used to account for the dependence of the data points (pixels in the data cube). Because it is hard to estimate how many pixels in the data cubes are relevant for the $\chi^{2}$ evaluation during the minimisation process, the calculations were made in two variants. In one case all pixels in the data cube were taken into account for the $\chi^{2}$ evaluation. In the other case a smaller number of data points was used by flagging those data points that show no emission in both bestfit model data cubes (the flat- and the rise model). The results (Table 5) show that, regardless of the method, the ratios of the $\chi^{2}$ probability densities favour the rise models.

In the case of NGC 2541 and UGC 3580, the results are robust against a possible degeneracy of rotation velocity and inclination: the rotation velocity rises where a slight increase in the inclination takes place. This is not the case for NGC 5204. Indeed, when keeping the rotation velocity fixed for inner and outer disks in the flat model, this change is partly compensated by a mean rise in the inclination of about $5.5 \mathrm{~km} \mathrm{~s}^{-1}$ in the outer parts. This change is significantly larger than an error that could arise from a TiRiFiC tilted-ring fit for a galaxy of similar properties as NGC 5204. In the course of testing TiRiFiC in Paper I, a synthetic observation of a down-sized idealised model of NGC 5204 containing realistic noise identical to that in the data cube of NGC 5204 was handed to an "observer". This observer then analysed this synthetic observation like a real observation (see test 13, Fig. 22 and Table 14 in the online version of Paper I). For the test, an artificial shift of the centre and the systemic velocity was added in order to enhance the difficulty for the observer to fit the observation. Nevertheless, the rms error in inclination taking the fainter regions into account amounted to $2^{\circ}$ (larger errors occurred only in the central few arcseconds). In Paper I it was shown that TiRiFiC generally produces reliable fits with respect to the inclination above $25^{\circ}$. Analysing symmetric warps in noisy data cubes, the error in inclination was lower than 2.8 in the well-defined reliable range in these tests. Thus, even ignoring the statistical evidence, it very unlikely that for NGC 5204 the rise in velocity is due to a degeneracy of inclination and rotation velocity.

These results indicate that a rise in the rotation velocity with increasing radius is needed in all three cases in order to explain the observations utilising a tilted-ring model. Except for NGC 5204, the change in rotation velocity could in principle be gradual as has been observed in other galaxies. Hence, in the case of NGC 2541 and UGC 3580, any connection to the warp has to be treated with caution. However, a comparison of the obtained changes with the rotation velocities measured for the inner disk shows the dynamical importance of this feature: the change in rotation velocity ranges from 6 per cent to 22 per cent (see Table 5).

\section{Non-circular motions and their possible influence on the results}

In the previous section evidence was given to the fact that the rotation velocity changes from the inner disk to the outer disks. This was done under the assumption of circularity of orbits inherent to the tilted-ring model. The detection of a change in this rotation velocity means that the physical conditions change from the inner to the outer disk. This holds at least for NGC 5204, where the data show most clearly that the change occurs abruptly at the transition radius. A change in the dynamics from the inner to the outer disk of NGC 5204 is thus very likely and suggested in the other two cases. Two explanations for the measured rise in the rotation velocity exist. The first possibility is that the change in the measured rotation velocity (derived using a tiltedring model) is due to the fact that indeed the local axisymmetric 
potential changes. This would then cause a real rise in the rotation velocity. A second possibility is that the local potential changes its symmetry, causing a different shape of the orbits. In that case the kinematics of the galaxies is only approximated by the tilted-ring model, and the changes in the symmetry are erroneously measured as a change in the tilted-ring rotation velocity.

In order to decide whether the shape of the orbits changes, it would be necessary to introduce more parameters in the tiltedring analysis (e.g. orbit ellipticity, radial and vertical motions, harmonic terms). These possibilities are not yet included in TiRiFiC; moreover, the degeneracies one introduces with such an approach have not been studied yet. A similar approach working with velocity fields (Schoenmakers et al. 1997) is even more prone to line-of-sight ambiguities than a tilted-ring modelling of velocity fields (Begemann 1987, see Paper I), especially in the presence of warps. It is therefore not a good option here.

The simplest approach has been used in order to get an impression of the influence of deviations in the kinematics of the galaxies from the symmetry inherent to the tilted-ring model. For each galaxy, the first-moment velocity map of the best-fit model data cubes was subtracted from the first-moment velocity map derived from the original observation (Fig. 13 in the online version of this paper). These difference maps are suited to spotting possible deviations in the fitted model kinematics from the observation. Such errors can arise because the model is not able to represent the galaxy kinematics; in Sect. 6 it has already been mentioned that, with the single-disk approach, at least the presence of the broadened profile at low intensities in the PV diagrams cannot be reproduced. For UGC 3580 and NGC 5204 and for the inner disk of NGC 2541, the largest systematic contribution to the deviations are of an antisymmetric ( $m=1)$ nature. In the region of the outer disk, NGC 2541 shows a systematic enhancement of the velocity difference (an $m=0$ feature).

Since the differences of the velocity maps show slight systematics, the possibility of an erroneous measurement cannot be excluded: the changes in the rotation velocities that were measured under the assumption of circular orbits possibly do not reflect a change in the axisymmetric potential. They might reflect a change of the orbit geometry without changing the character of the radial potential.

\section{Summary of the results and discussion}

Within the errors the sample galaxies follow the rules for the behaviour of warps laid down by Briggs (1990) up to a certain radius. In addition, the following features are found to be present in the examined $\mathrm{H}$ I disks:

i) The galaxies show an outer comparably planar regime, so they have an inner flat disk in which most of the visible stars reside and an outer flat disk. Both regions are connected at the transition radius, at which the disk orientation changes rapidly.

ii) In two cases (NGC 2541 and NGC 5204), the H I surface density profile shows a drop at the transition radius, which is seen with less significance in the case of NGC 3580. Beyond the transition radius, the H I surface density is falling only slowly with radius or even remains constant.

Briggs' Briggs (1990) fourth rule is a direct consequence of a flat outer disk. It is found that:

iii) Under the assumption of circularity of orbits, the rotation curve changes from the inner part of the galaxy to the outer one.
Statistical evidence for this change is presented in all three cases. In the case of NGC 5204, the change in rotation velocity clearly shows up as a sudden increase at the transition radius. In the other two cases, the rotation curve is compatible with a gradual rise.

iv) In all three cases, a slowly rotating extraplanar gas component is detected.

This gas component shows up in a comparison of measurements with single-disk model data cubes and as broadened profiles in the PV diagrams, in NGC 5204 even gas at forbidden velocities is seen. At this stage, it is not clear whether the presence of this gas component is connected to the warping phenomenon, but the coincidence is striking (see below).

The studied galaxies have been selected to be highly symmetric, and it could be shown that they maintain their symmetry to very large radii. The large orbital times at these radii (see Table 1) imply that the warps in the galaxies studied are either long-lived stationary structures or very slowly evolving transitional phenomena. While the number of galaxies presented in this work is by far too small to draw any general conclusions, the presence of the reported features in possibly all three cases raises nevertheless the question of whether they are immanent to a certain type of long-lived symmetric warp. Searching the literature, one can find several reports indicating that the same warp structure in other disk galaxies (NGC 628, NGC 1058, NGC 2841, NGC 3344, NGC 3718, NGC 5055, and M 83: Briggs 1990; Kuijken 1991; but see also Battaglia et al. 2006 for NGC 5055. M 33: Corbelli \& Schneider 1997. Milky Way: de Boer et al. 2005; Dedes et al. 2005; Levine et al. 2006, a detailed discussion can be found in Józsa 2006).

The question arises as to whether these findings can help put constraints on the underlying warp formation process. Proposed scenarios mainly have to face the fact that in the examined galaxies with the existence of the flat outer disk rule 3 of Briggs (1990) does not hold at large radii, where the LON stops to show a clear tendency to increase in the direction of rotation. As already discussed in Sect. 1, scenarios that connect warping with cosmic infall are presently the most successful ones. In these models, the tendency of the LON to wind up towards larger radii is explained by an outwards decreasing torque on the disk. Without the presence of an agent that acts in a reverse manner at large radii, these models must fail to reproduce a flat region in the galactic disk, as is the case in Debattista \& Sellwood (1999) and Bailin \& Steinmetz (2003).

As discussed in Sect. 1, the simulations of Shen \& Sellwood (2006), in which cosmic infall was simulated by actively injecting particles on a fixed orbit inclined with respect to the disk, show that a realistic warp could be reproduced, given that a further increase in sensitivity would clearly show a regressing windup of the LON at even larger radii. In this model, the artificial outer ring imposes an additional outwards increasing torque on the disk that in a twist region balances up the (prograde) differential precession due to the outwards decreasing torque from the disk itself. While a direct detection of such a twist cannot be reported on here (see Figs. 4 and 10 in the online version), given the presence of sufficiently large errors, such a feature can be identified as a (comparably) constant orientation within the reach of the twist radius. Therefore a scenario with an agent that produces an outwards increasing torque at large radii could possibly be compatible with the detection of an outer flat region in a galaxy disk, provided, a model setup can be found for which the region is very large within which the spirality of the 
LON changes. In the specific models shown in Shen \& Sellwood (2006) however, this does not seem to be the case.

A remarkable incidence is the rise of the rotation curve towards the warp region, which could be explained in principle by the presence of a ring of debris at this position. In order to exert the same torque and at the same time be the cause for a rise in the rotation velocity, at smaller radii such a ring would have considerably less relative mass than the one used by Shen \& Sellwood (2006) (2.5 disk masses). In that case, however, the treatment of the torus as a passive external agent for warp formation would not be valid anymore, as its moment of inertia would be reduced significantly, and the torque exerted from the disk on the torus would be significantly larger. The presence of the extraplanar gas possibly supports the accretion scenario: it is known that the kinematical structure of extraplanar gas detected in other galaxies cannot be explained by internal processes alone (Collins et al. 2002; Fraternali \& Binney 2006), but an external supply of (primordial) gas with low angular momentum is needed. One might therefore speculate that the slowly rotating extraplanar gas component is acquired in the same accretion event that excites the warp. However, in order to follow up on this, detailed studies are necessary and will be presented in a forthcoming paper.

The velocity rise can also indicate a change of the DM halo shape. In response to a massive torus the halo is expected to become increasingly flattened outwards (see also Bailin et al. 2005) and the rotation velocity will increase in response. This issue will be followed up on in a case study of the spiral galaxy NGC 755 presented in Paper III of this series.

The detection of the flatness of the outer disks in the observed galaxies is possibly compatible with a very slowly evolving temporary structure. The extent of the flat outer disks, however, is rather indicative of a long-lived stationary structure, as within the errors no differential precession seems to be present over a wide range of radii.

In their simulations, (Shen \& Sellwood 2006) find that, after the removal of the forcing agent that ignites the warp in their simulations, the warp maintains its amplitude while the disk tends to wind up slowly at intermediate radii (see also Binney et al. 1998). But as the inner (aligned) disk-DM halo system and the outer (flattened) DM halo exchange angular momentum very ineffectively, their mutual inclination decreases very slowly. The question remains as to how a thin, gaseous disk at large radii reacts to the forces stemming from the outer DM halo and how it evolves in the long term.

In simulations using a standard cosmological setup, following the time evolution of a disk-DM halo system after a last merger event, Bailin et al. (2005) find the same intrinsic misalignment of the DM halo to be present. Within the disk, disk and halo have the same orientation, while at larger radii the (flattened) halo is tilted with respect to the inner disk-halo system. The mutual inclination of both systems stays constant until the end of the simulation is reached (after $2.2 \mathrm{Gyr}$ ). If a thin gaseous disk traced the outer flat halo (Bailin et al. 2005), the whole disk structure would show a warp structure with an outer flat disk as described here.

Thus, the warps of the galaxies studied in this paper are possibly tracing a long-lived, rather stationary structure, consisting of an inner, flattened disk-DM halo system and an outer misaligned flattened DM halo, which precess slowly about each other, the amplitude of the misaligned decreasing very slowly due to an ineffective exchange of angular momentum between the inner and outer portion of the DM halo.

Acknowledgements. I am deeply indebted to Tom Oosterloo and Uli Klein for their personal and scientific support when conducting the underlying Ph.d. thesis project and for their important comments on this paper. I thank Franz Kenn for many fruitful discussions. I thank Peter Kalberla for comments on this paper. I thank the referee, Rob Swaters, for numerous critical suggestions enhancing the quality of the paper. The underlying thesis project was partly financed by the Deutsche Forschungsgemeinschaft in the framework of the Graduiertenkolleg 787 "Galaxy Groups as Laboratories for Baryonic and Dark Matter". It was also partly financed by the University of Bonn in the framework of the "Research Group Bonn: Dark Matter and Dark Energy". This research has made use of the NASA/IPAC Extragalactic Database (NED) and the HyperLeda database.

\section{References}

Avner, E. S., \& King, I. R. 1967, AJ, 72, 650

Bailin, J. 2003, ApJ, 583, L79

Bailin, J., \& Steinmetz, M. 2003, Ap\&SS, 284, 701

Bailin, J., Kawata, D., Gibson, B. K., et al. 2005, ApJ, 627, L17

Battaglia, G., Fraternali, F., Oosterloo, T., \& Sancisi, R. 2006, A\&A, 447, 49

Battaner, E., \& Jimenez-Vicente, J. 1998, A\&A, 332, 809

Battaner, E., Florido, E., \& Sánchez-Saavedra, M. L. 1990a, A\&A, 236, 1

Battaner, E., Florido, E., \& Sánchez-Saavedra, M. L. 1990b, in Galactic and Intergalactic Magnetic Fields, ed. R. Beck, P. P. Kronberg, \& R. Wielebinski (Dordrecht: Kluwer), IAU Symp., 140, 504

Begemann, K. G. 1987, Ph.D. Thesis

Binney, J. 1978, MNRAS, 183, 779

Binney, J. 1992, ARA\&A, 30, 51

Binney, J., \& Merrifield, M. 1998, Galactic astronomy (Princeton, NJ: Princeton University Press)

Binney, J., Jiang, I., \& Dutta, S. 1998, MNRAS, 297, 1237

Bosma, A. 1978, Ph.D. Thesis, Univ. Groningen

Brada, R., \& Milgrom, M. 2000, ApJ, 531, L21

Briggs, F. H. 1990, ApJ, 352, 15

Briggs, F., \& Kamphuis, J. 1991, in Warped Disks and Inclined Rings around Galaxies, ed. S. Casertano, P. Sackett, \& F. Briggs (Cambridge: CUP), 190

Burke, B. F. 1957, AJ, 62, 90

Cabrera-Lavers, A., \& Garzón, F. 2004, AJ, 127, 1386

Chapelon, S., Contini, T., \& Davoust, E. 1999, A\&A, 345, 81

Christodoulou, D. M., \& Tohline, J. E. 1986, ApJ, 307, 449

Coccato, L., Corsini, E. M., Pizzella, A., et al. 2004, A\&A, 416, 507

Collins, J. A., Benjamin, R. A., \& Rand, R. J. 2002, ApJ, 578, 98

Corbelli, E., \& Schneider, S. E. 1997, ApJ, 479, 244

Cox, A. L., Sparke, L. S., van Moorsel, G., \& Shaw, M. 1996, AJ, 111, 1505

de Boer, W., Sander, C., Zhukov, V., Gladyshev, A. V., \& Kazakov, D. I. 2005, A\&A, 444, 51

de Vaucouleurs, G., de Vaucouleurs, A., Corwin, H. G., et al. 1991, Third Reference Catalogue of Bright Galaxies (Berlin Heidelberg New York: Springer)

Debattista, V. P., \& Sellwood, J. A. 1999, ApJ, 513, L107

Dedes, L., Kalberla, P. M. W., Arnal, E. M., et al. 2005, in Extra-Planar Gas, ed. R. Braun, ASP Conf. Ser., 331, 75

Dekel, A., \& Shlosman, I. 1983, in Internal Kinematics and Dynamics of Galaxies, ed. E. Athanassoula (Dordrecht: Kluwer), IAU Symp., 100, 187 Dubinski, J., \& Kuijken, K. 1995, ApJ, 442, 492

Elwert, G., \& Hablick, D. 1965, Zeitschrift für Astrophysik, 61, 273

Ferrarese, L., Bresolin, F., Kennicutt, R. C., et al. 1998, ApJ, 507, 655

Florido, E., Prieto, M., Battaner, E., et al. 1991, A\&A, 242, 301

Fraternali, F., \& Binney, J. J. 2006, MNRAS, 366, 449

Fraternali, F., Oosterloo, T., \& Sancisi, R. 2004, Ap\&SS, 289, 377

Freedman, W. L., Madore, B. F., Gibson, B. K., et al. 2001, ApJ, 553, 47

Gao, L., \& White, S. D. M. 2006, MNRAS ArXiv Astrophysics e-prints, submitted

García-Ruiz, I., Sancisi, R., \& Kuijken, K. 2002, A\&A, 394, 769

Garrido, O., Marcelin, M., \& Amram, P. 2004, MNRAS, 349, 225

Hofner, P., \& Sparke, L. S. 1994, ApJ, 428, 466

Hunter, C., \& Toomre, A. 1969, ApJ, 155, 747

Jiang, I., \& Binney, J. 1999, MNRAS, 303, L7

Józsa, G. I. G. 2006, Ph.D. Thesis, Univ. Bonn, available at

http://hss.ulb.uni-bonn.de/diss_online/math_nat_fak/

2006/jozsa_gyula/index.htm

Józsa, G. I. G., Kenn, F., Klein, U., \& Oosterloo, T. A. 2007a, A\&A, 468, 731 (Paper 1)

Józsa, G. I. G., Niemczyk, C., Klein, U., \& Oosterloo, T. A. 2007b, in New Astron. Rev., The Fate of the Gas in Galaxies, ed. R. Morganti, T. A. Oosterloo, M. Villar-Martin, \& J. H. van Gorkom, 51, 273

Kahn, F. D., \& Woltjer, L. 1959, ApJ, 130, 705

Karachentsev, I. D., Kopylov, A. I., \& Kopylova, F. G. 1994, Bull. Special Astrophys. Obs., 38, 5 
Karachentsev, I. D., Sharina, M. E., Dolphin, A. E., et al. 2003, A\&A, 398, 467 Kerr, F. J. 1957, AJ, 62, 93

Kuijken, K. 1991, ApJ, 376, 467

López-Corredoira, M., Betancort-Rijo, J., \& Beckman, J. E. 2002, A\&A, 386, 169

Levine, E. S., Blitz, L., \& Heiles, C. 2006, ApJ, 643, 881

Lynden-Bell, D. 1965, MNRAS, 129, 299

Nelson, R. W., \& Tremaine, S. 1995, MNRAS, 275, 897

Ostriker, E. C., \& Binney, J. J. 1989, MNRAS, 237, 785

Paturel, G. 1989, Catalogue of principal galaxies (PGC) (Lyon: Base de Données Extragalactiques, Observatoire de Lyon)

Paturel, G., Petit, C., Prugniel, P., et al. 2003, A\&A, 412, 45

Reshetnikov, V., \& Combes, F. 1998, A\&A, 337, 9

Revaz, Y., \& Pfenniger, D. 2001, A\&A, 372, 784

Revaz, Y., \& Pfenniger, D. 2004, A\&A, 425, 67

Rogstad, D. H., Lockart, I. A., \& Wright, M. C. H. 1974, ApJ, 193, 309

Sakai, S., Mould, J. R., Hughes, S. M. G., et al. 2000, ApJ, 529, 698

Sánchez-Saavedra, M. L., Battaner, E., Guijarro, A., López-Corredoira, M., \& Castro-Rodríguez, N. 2003, A\&A, 399, 457

Sánchez-Salcedo, F. J. 2004, J. Korean Astron. Soc., 37, 205

Sánchez-Salcedo, F. J. 2006, MNRAS, 365, 555

Sault, R. J., Teuben, P. J., \& Wright, M. C. H. 1995, in Astronomical Data Analysis Software and Systems IV (San Francisco: ASP), ASP Conf. Ser., 77,433

Schoenmakers, R. H. M., Franx, M., \& de Zeeuw, P. T. 1997, MNRAS, 292, 349
Schwarzkopf, U., \& Dettmar, R.-J. 2001, A\&A, 373, 402

Shang, Z., Brinks, E., Zheng, Z., et al. 1998, ApJ, 504, L23

Sharma, S., \& Steinmetz, M. 2005, ApJ, 628, 21

Shen, J., \& Sellwood, J. A. 2006, MNRAS, 370, 2

Sicotte, V., \& Carignan, C. 1997, AJ, 113, 609

Sicotte, V., Carignan, C., \& Durand, D. 1996, AJ, 112, 1423

Sparke, L. S. 1984, ApJ, 280, 117

Sparke, L. S. 1985, in The Milky Way Galaxy, ed. H. van Woerden, R. J. Allen,

\& W. B. Burton (Dordrecht: Kluwer), IAU Symp., 106, 499

Sparke, L. S., \& Casertano, S. 1988, MNRAS, 234, 873

Swaters, R. A. 2001, in Galaxy Disks and Disk Galaxies, ed. J. G. Funes, \&

E. M. Corsini (San Francisco: ASP), ASP Conf. Ser., 230, 545

Swaters, R. A., \& Balcells, M. 2002, A\&A, 390, 863

Swaters, R. A., Madore, B. F., \& Trewhella, M. 2000, ApJ, 531, L107

Swaters, R. A., Madore, B. F., van den Bosch, F. C., \& Balcells, M. 2003, ApJ, 583,732

Toomre, A. 1983, in Internal Kinematics and Dynamics of Galaxies, ed. E. Athanassoula (Dordrecht: Kluwer), IAU Symp., 100, 177

Tubbs, A. D., \& Sanders, R. H. 1979, ApJ, 230, 736

van der Hulst, J. M., van Albada, T. S., \& Sancisi, R. 2001, in Gas and Galaxy Evolution, ed. J. E. Hibbard, M. Rupen, \& J. H. van Gorkom (San Francisco: ASP), ASP Conf. Ser., 240, 451

Weinberg, M. D. 1998, MNRAS, 299, 499

Weinberg, M. D., \& Blitz, L. 2006, ApJ, 641, L33 
G. I. G. Józsa: Kinematic modelling of disk galaxies. II., Online Material p 1

\section{Online Material}


G. I. G. Józsa: Kinematic modelling of disk galaxies. II., Online Material p 2

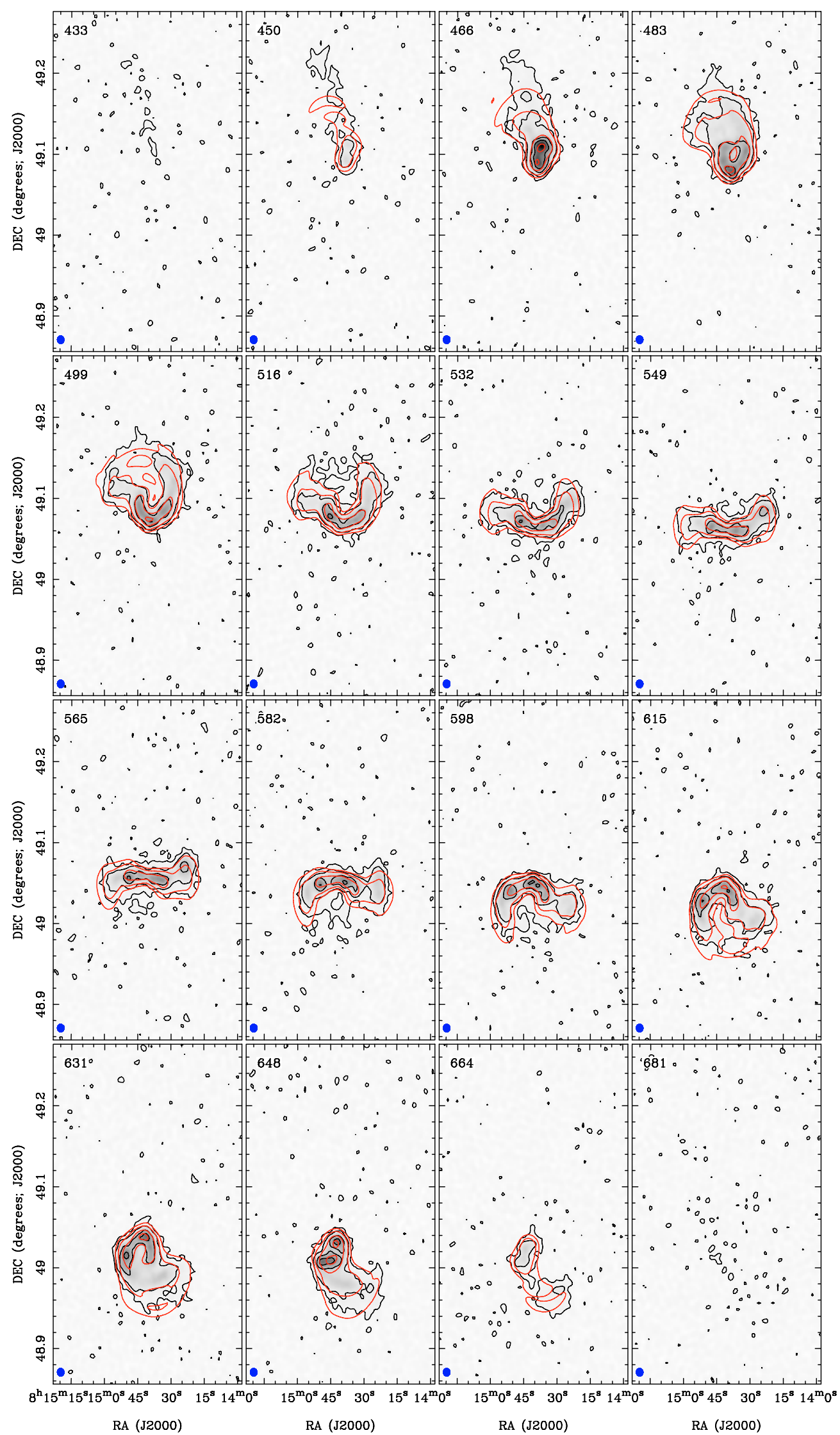

Fig. 5. Selected images from the low-resolution data cubes overlaid with contours of the original data set (blue) and the final model data cube (pink). The numbers on the upper left give the heliocentric radio velocity in $\mathrm{km} \mathrm{s}^{-1}$. The blue dot represents the clean beam. NGC 2541 . The contours represent the $1,4,16,32 \mathrm{mJy} /$ beam levels. 
G. I. G. Józsa: Kinematic modelling of disk galaxies. II., Online Material p 3

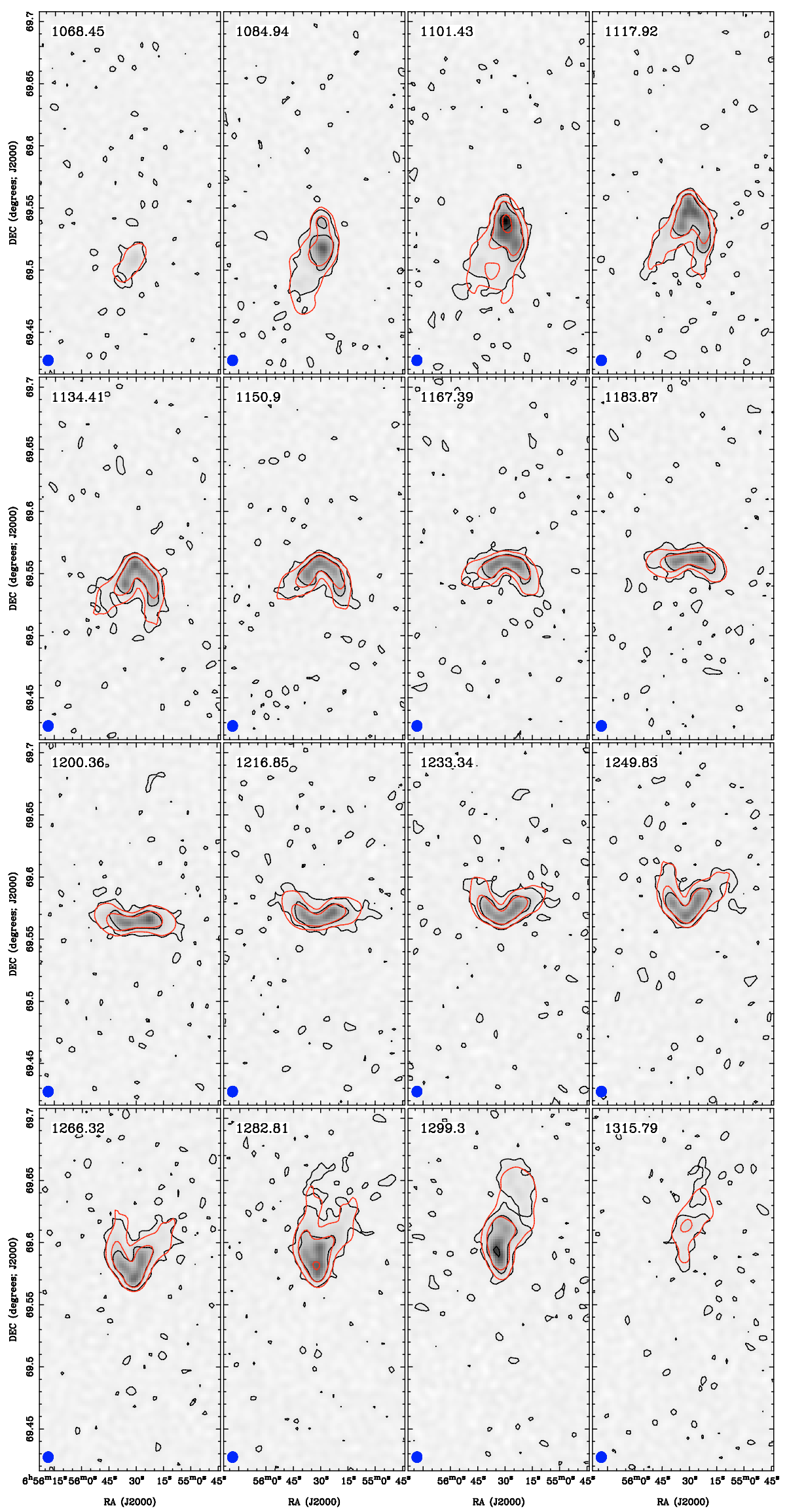

Fig. 5. continued. UGC 3580 . The contours represent the $1,6,24 \mathrm{mJy} /$ beam levels. 
G. I. G. Józsa: Kinematic modelling of disk galaxies. II., Online Material $p 4$

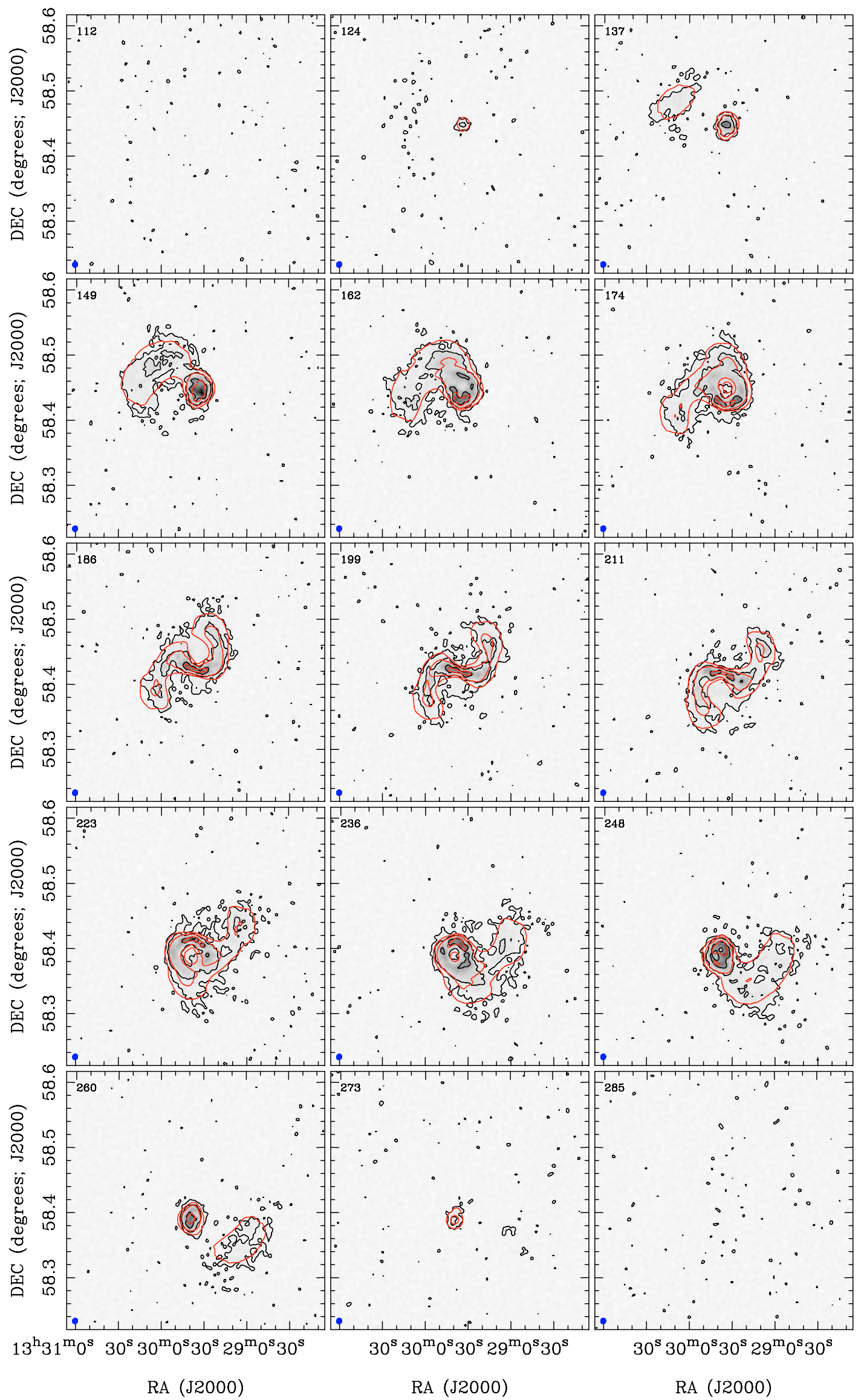

Fig. 5. continued. NGC 5204. The contours represent the 1, 4, $16 \mathrm{mJy} /$ beam levels. 
G. I. G. Józsa: Kinematic modelling of disk galaxies. II., Online Material p 5

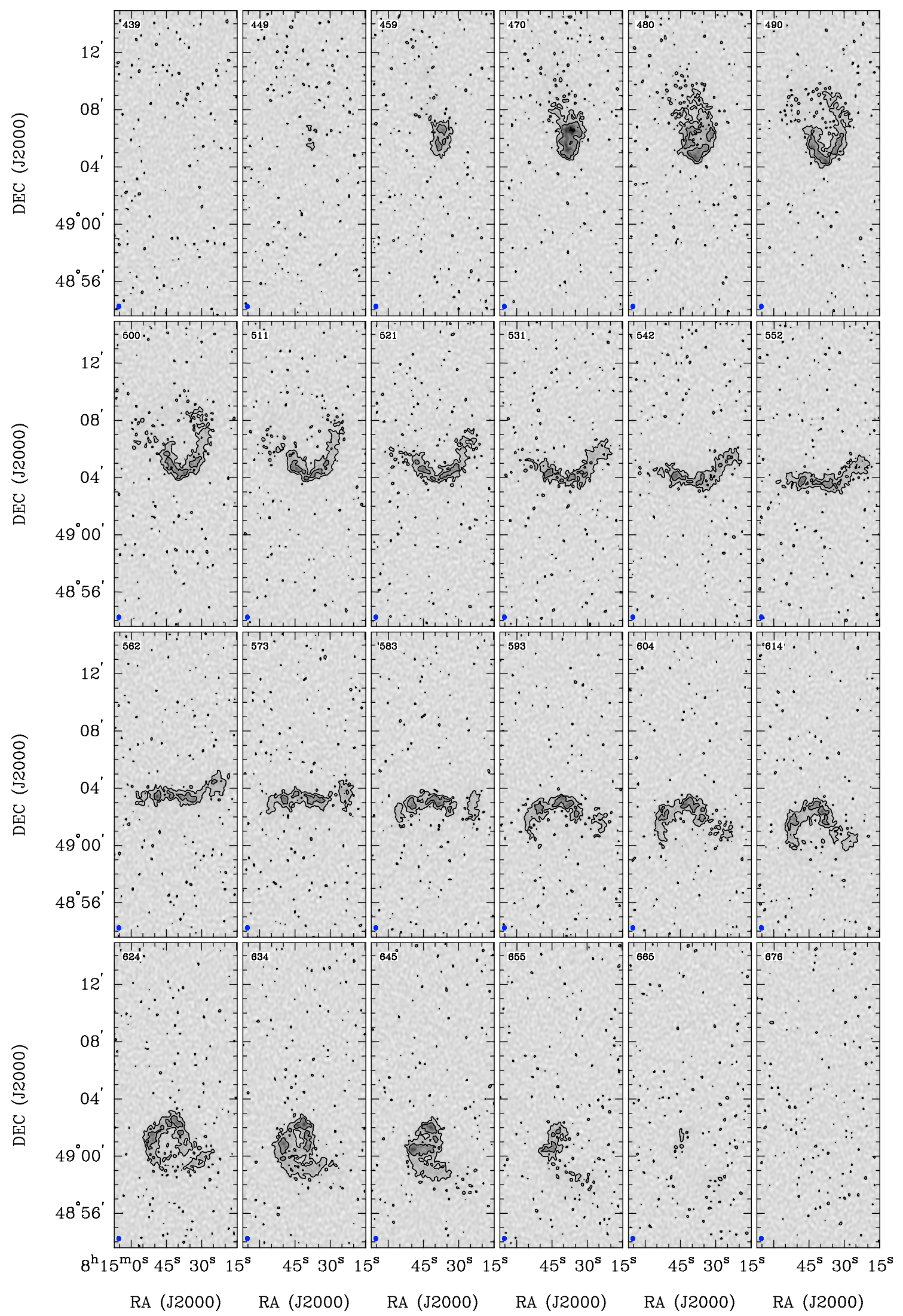

Fig. 6. Selected images from the high-resolution data cubes (channel resolution of $2.06 \mathrm{~km} \mathrm{~s}^{-1}$, uniform weighting, all visibilities used). The numbers in the upper left give the heliocentric radio velocity in $\mathrm{km} \mathrm{s}^{-1}$. The blue dot represents the clean beam. NGC 2541 . The contours represent the 2,6,18 $\mathrm{mJy} /$ beam levels. 
G. I. G. Józsa: Kinematic modelling of disk galaxies. II., Online Material p 6

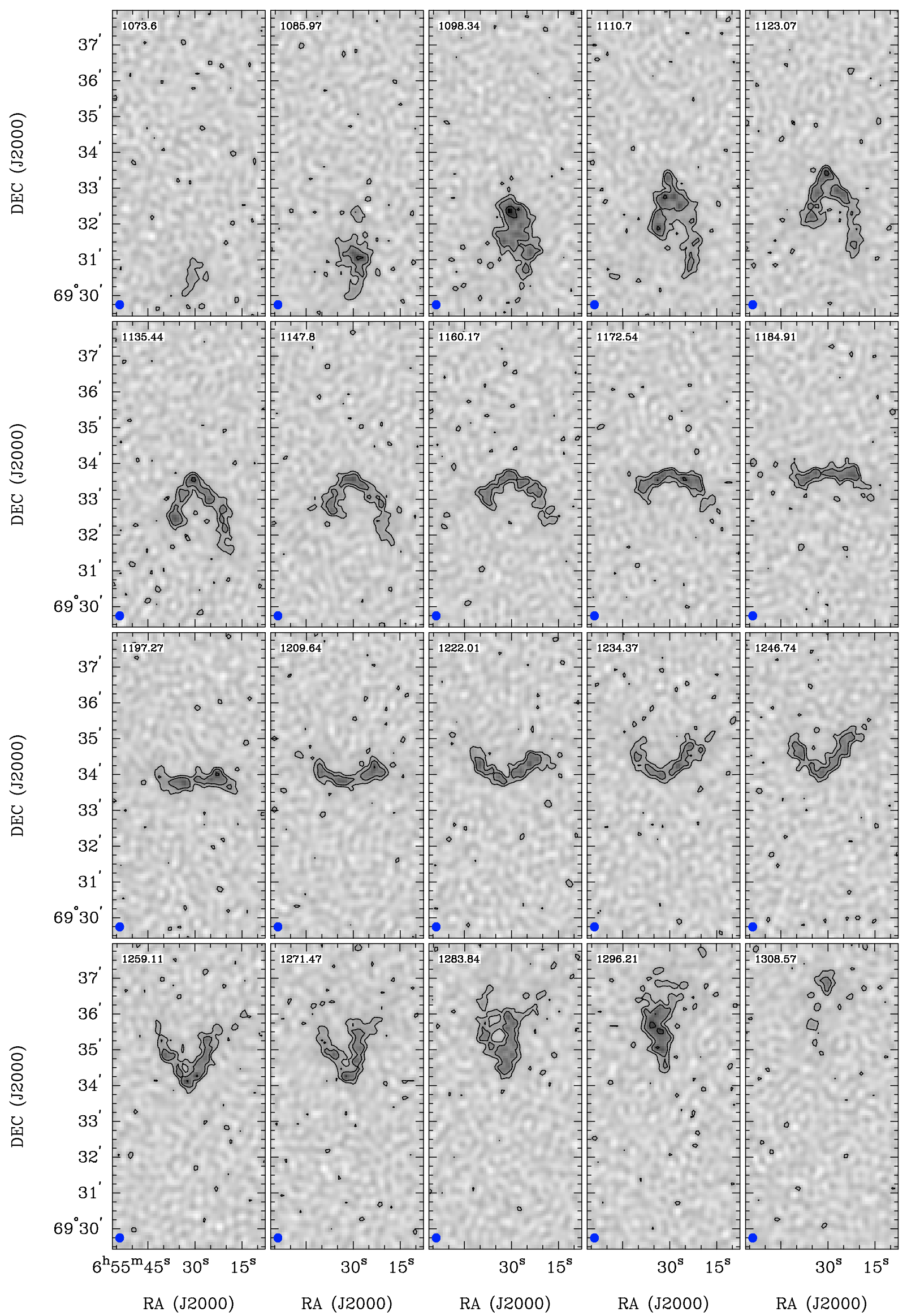

Fig. 6. continued. UGC 3580 . The contours represent the 2, 8, $16 \mathrm{mJy} /$ beam levels. 
G. I. G. Józsa: Kinematic modelling of disk galaxies. II., Online Material $p 7$

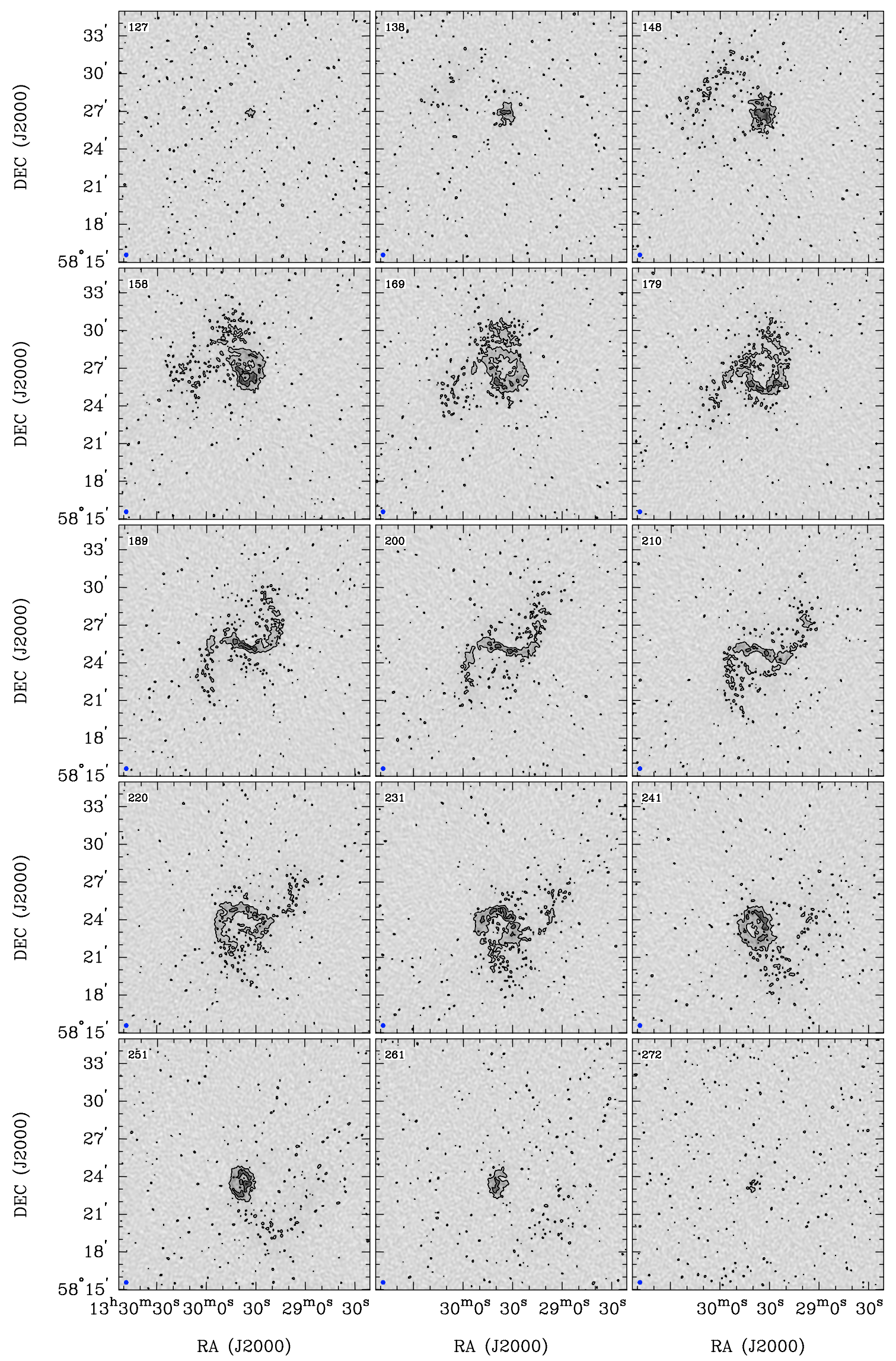

Fig. 6. continued. NGC 5204. The contours represent the $1.5,6 \mathrm{mJy} /$ beam levels. 
G. I. G. Józsa: Kinematic modelling of disk galaxies. II., Online Material p 8
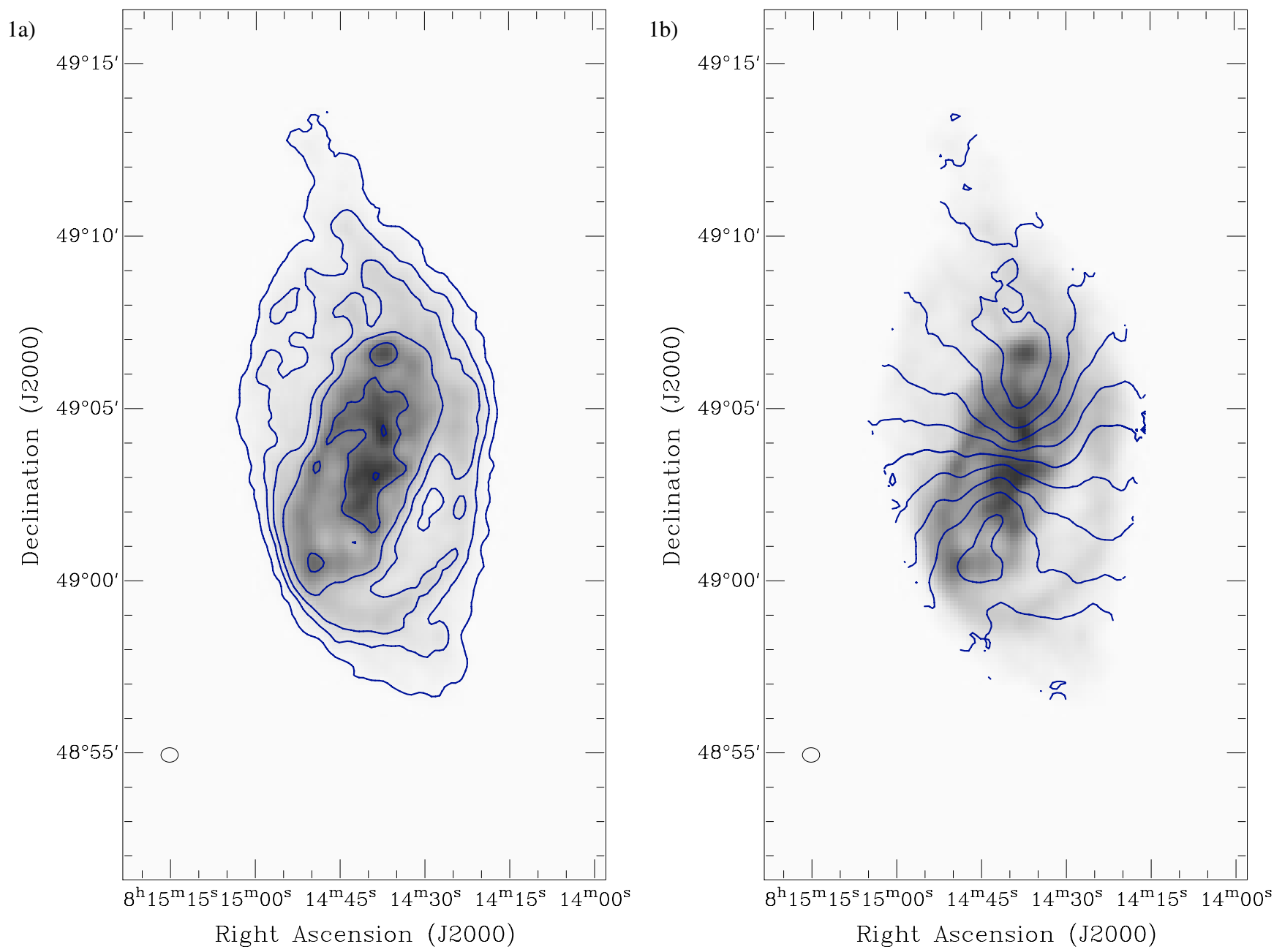

Fig. 7. Total intensity maps (a), left) and first-moment velocity fields (b), right) derived from the low-resolution data cubes overlaid on total intensity grey-scale maps. The ellipse in the lower left corner represents the clean beam. NGC 2541. The contours represent the $5,20,40,80,160,240 \times 10^{19}$ atoms cm$~^{-2}$ levels and the $V_{\text {sys }} \pm 0,20,40,60,80,100 \mathrm{~km} \mathrm{~s}^{-1}$ levels, respectively (see Table 1). The approaching side is oriented NE. 
G. I. G. Józsa: Kinematic modelling of disk galaxies. II., Online Material $p 9$
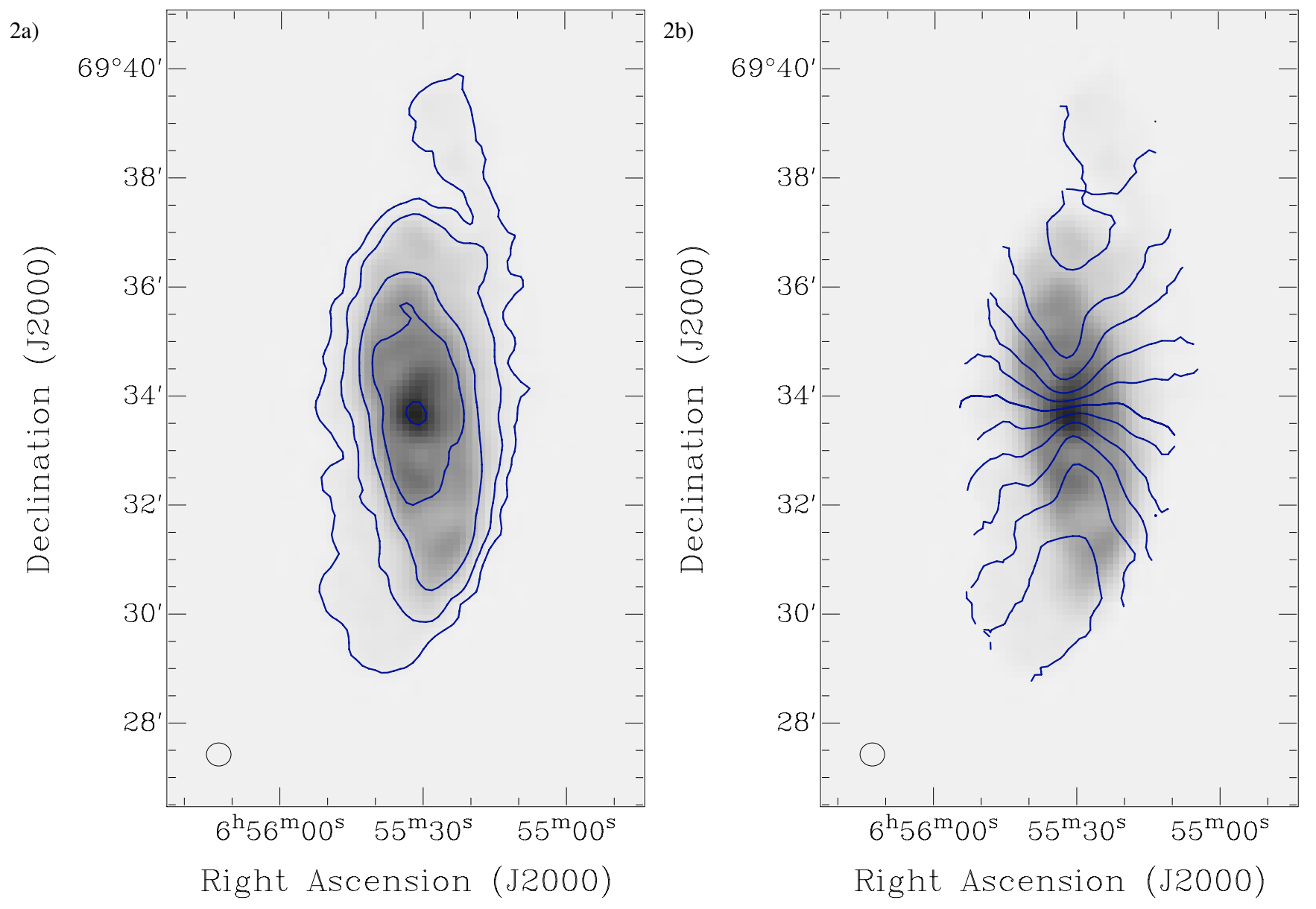

Fig. 7. continued. UGC 3580 . The contours represent the $6,19,57,114,228 \times 10^{19}$ atoms cm ${ }^{-2}$ levels and the $V_{\text {sys }} \pm 0,20,40,60,80,100 \mathrm{~km} \mathrm{~s}^{-1}$ levels, respectively (see Table 1). The approaching side is oriented SE.
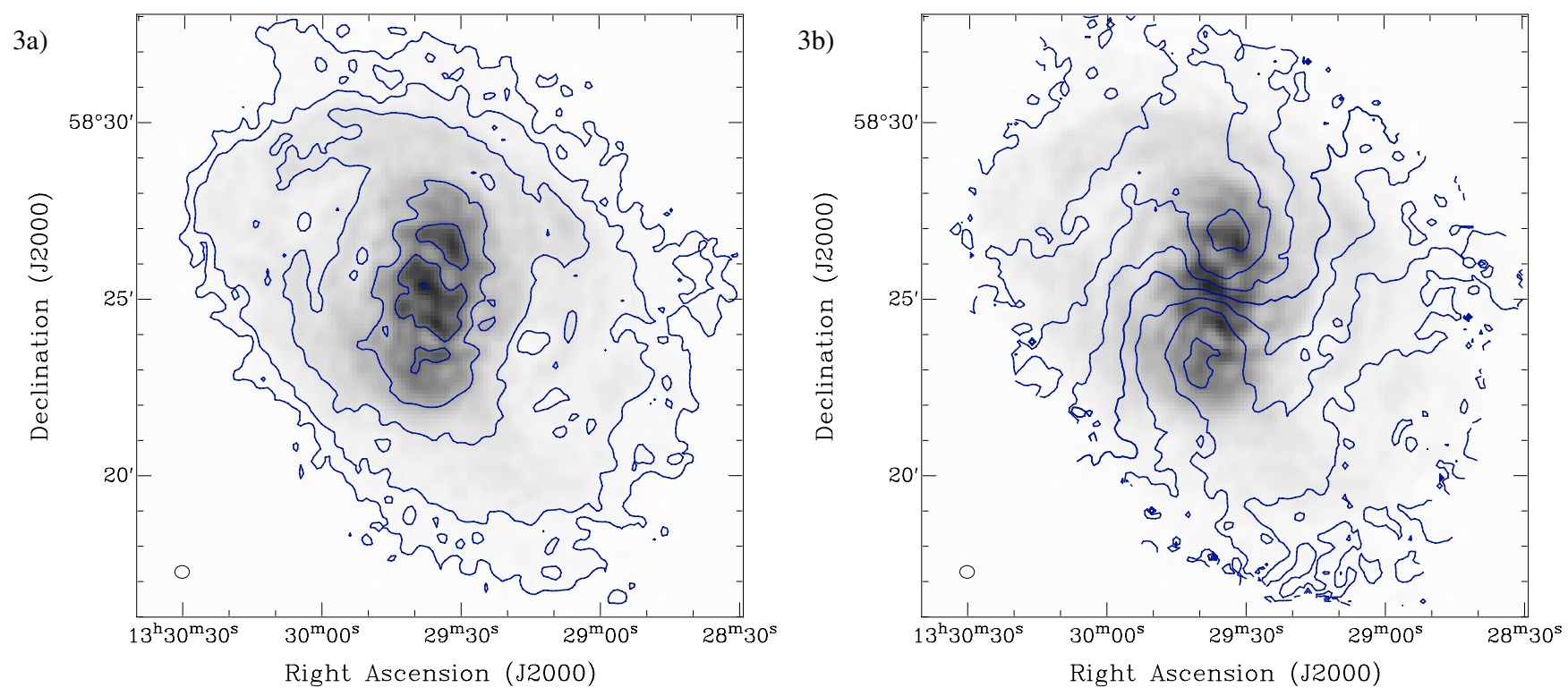

Fig. 7. continued. NGC 5204. The contours represent the $2.5,8,25,75,145,225 \times 10^{19}$ atoms cm ${ }^{-2}$ levels and the $V_{\text {sys }} \pm 0,15,30,45,60 \mathrm{~km} \mathrm{~s}^{-1}$ levels, respectively (see Table 1). The approaching side is oriented NE. 
G. I. G. Józsa: Kinematic modelling of disk galaxies. II., Online Material p 10
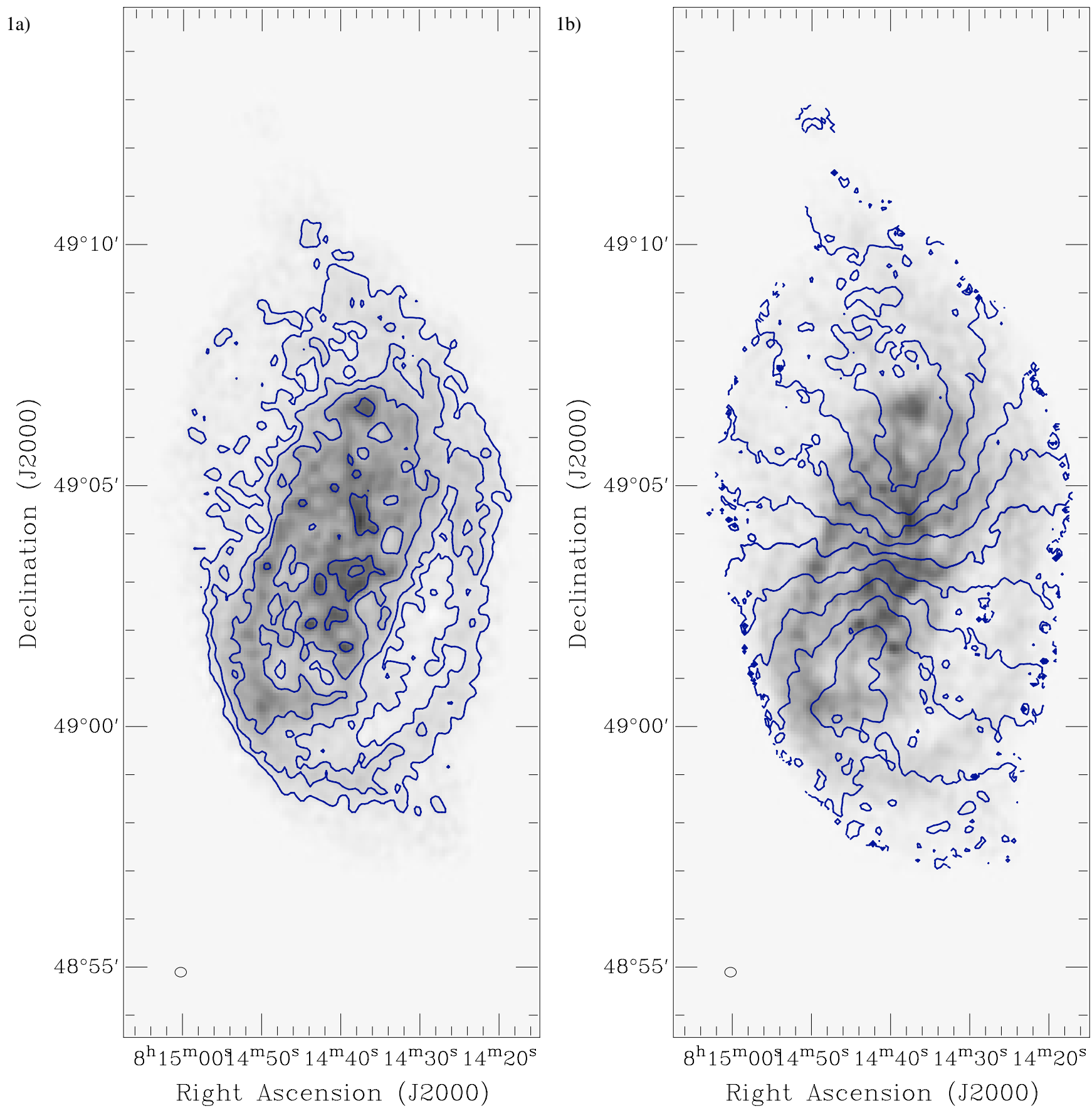

Fig. 8. Total intensity maps (a), left) and first moment velocity fields (b), right) derived from the high-resolution data cubes overlaid on total intensity grey-scale maps. The ellipse in the lower left corner represents the clean beam. NGC 2541 . The contours represent the $29,58,115,230,345 \times 10^{19}$ atoms cm$~^{-2}$ levels and the $V_{\text {sys }} \pm 0,20,40,60,80,100 \mathrm{~km} \mathrm{~s}^{-1}$ levels, respectively (see Table 1 ). The approaching side is oriented NE. 
G. I. G. Józsa: Kinematic modelling of disk galaxies. II., Online Material p 11
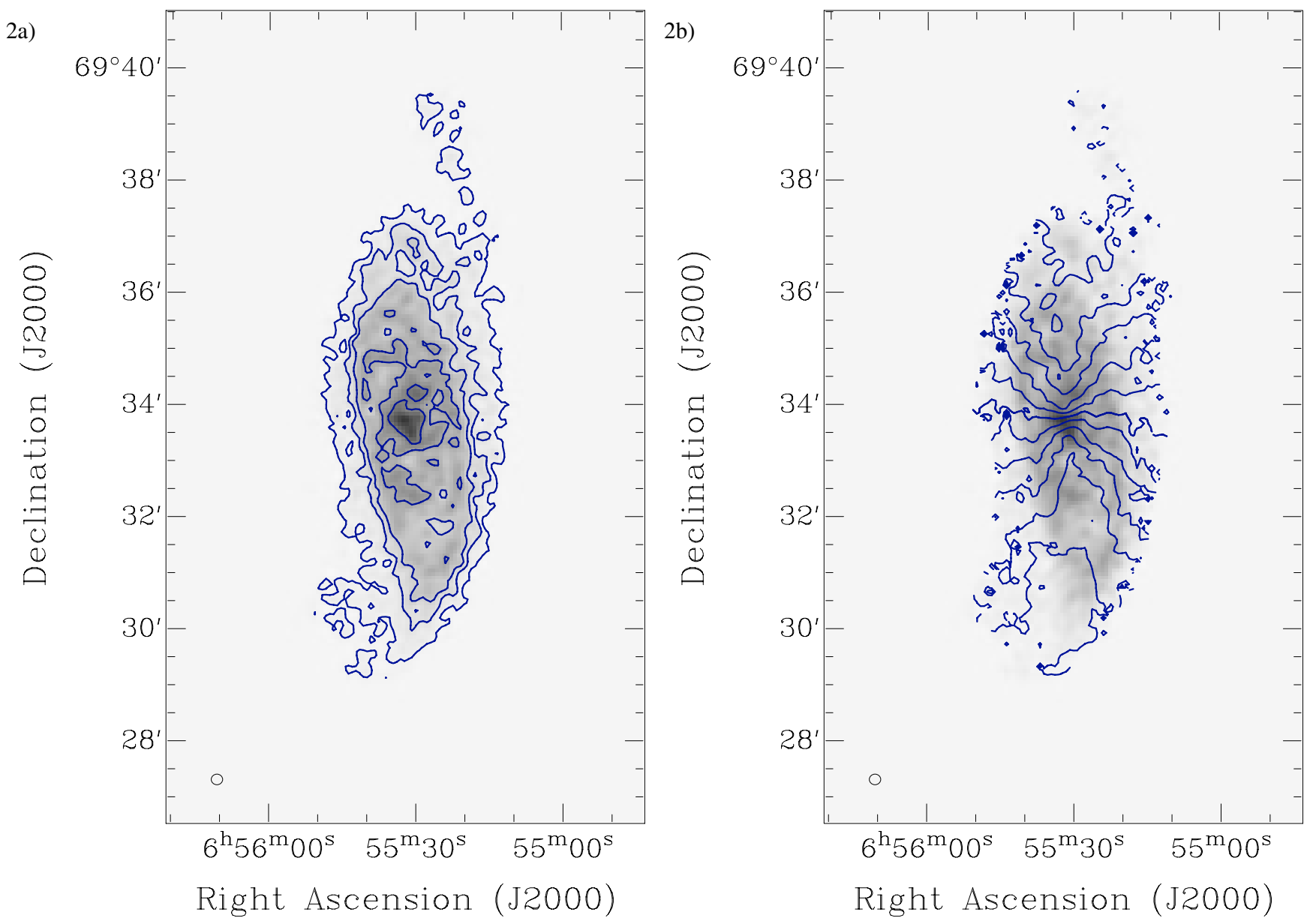

Fig. 8. continued. UGC 3580 . The contours represent the $8,35,69,139,208 \times 10^{19}$ atoms cm ${ }^{-2}$ levels and the $V_{\text {sys }} \pm 0,20,40,60,80,100 \mathrm{~km} \mathrm{~s}^{-1}$ levels, respectively (see Table 1). The approaching side is oriented SE.
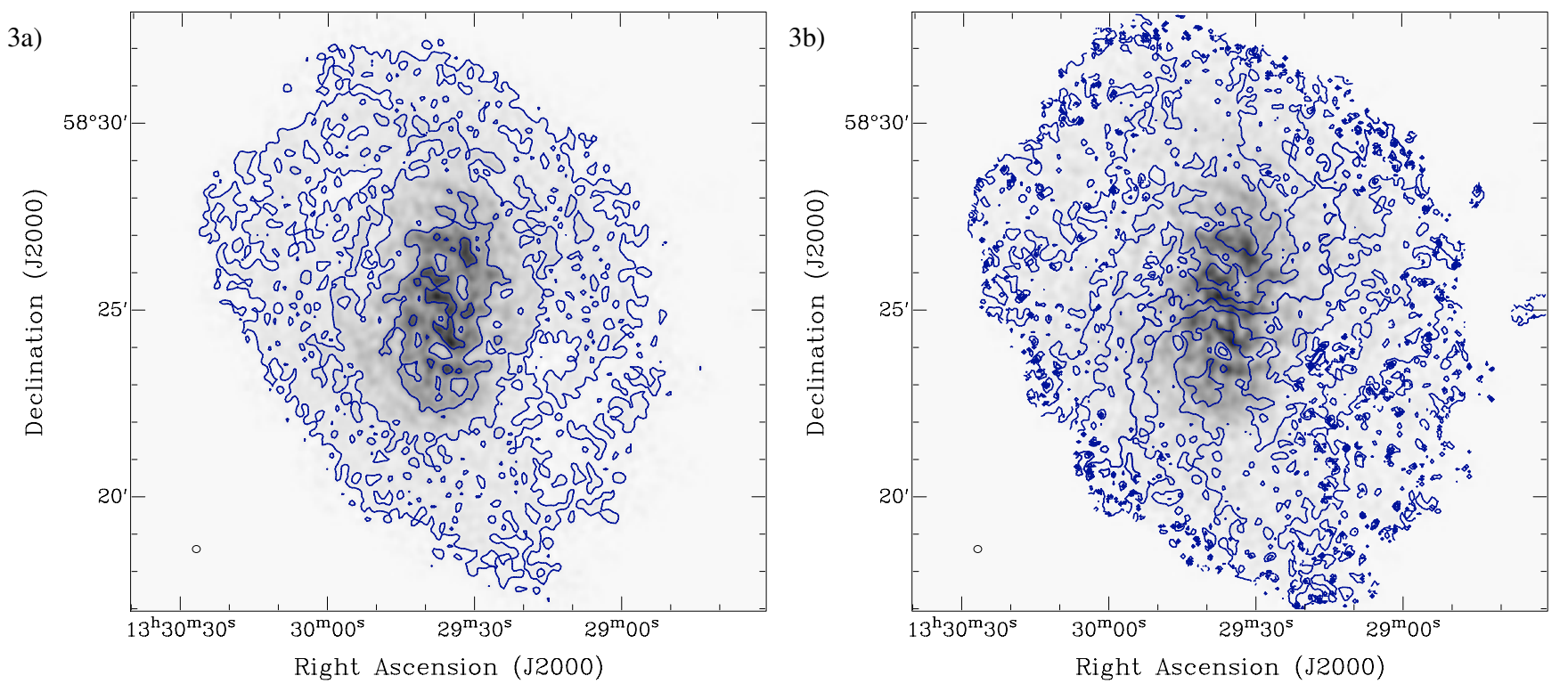

Fig. 8. continued. NGC 5204. The contours represent the $11,32,97,194 \times 10^{19}$ atoms cm $\mathrm{cm}^{-2}$ levels and the $V_{\text {sys }} \pm 0,15,30,45,60 \mathrm{~km} \mathrm{~s}^{-1}$ levels, respectively (see Table 1). The approaching side is oriented NE. 
G. I. G. Józsa: Kinematic modelling of disk galaxies. II., Online Material p 12

Table 6. Radially dependent best-fit parameters for the examined galaxies. (1) Radius ("). (2) Radius (kpc, according to Table 1). (3) Error of $r_{\mathrm{t}}$ $(\mathrm{kpc})$ (4) Face-on surface brightness $\left(\mathrm{Jy} \mathrm{km} \mathrm{s}^{-1}\right)$. (5) Error of $I_{\mathrm{tot}, \mathrm{f}}\left(\mathrm{Jy} \mathrm{km} \mathrm{s}^{-1}\right)$. (6) Face-on H I column density $\left(10^{19}\right.$ atoms $\left.\mathrm{cm}^{-2}\right)$. (7) Error of $N_{\mathrm{H} \text { I }}$ $\left(10^{19}\right.$ atoms cm $\left.{ }^{-2}\right)$. (8) Face-on surface density $\left(M_{\odot} \mathrm{pc}^{-2}\right)$. (9) Error of $\sigma\left(M_{\odot} \mathrm{pc}^{-2}\right)$. (10) Rotation velocity $\left(\mathrm{km} \mathrm{s}^{-1}\right)$. (11) Error of $V_{\text {rot }}\left(\mathrm{km} \mathrm{s}^{-1}\right)$.

\begin{tabular}{rrrrrrrrrrr}
\hline \hline$r_{\mathrm{p}}$ & $\begin{array}{r}r_{\mathrm{t}} \\
(1)\end{array}$ & $\begin{array}{r}\Delta r_{\mathrm{t}} \\
(3)\end{array}$ & $\begin{array}{r}I_{\text {tot,f }} \\
(4)\end{array}$ & $\begin{array}{r}\Delta I_{\text {tot,f }} \\
(5)\end{array}$ & $\begin{array}{r}N_{\mathrm{HI}} \\
(6)\end{array}$ & $\begin{array}{r}\Delta N_{\mathrm{HI}} \\
(7)\end{array}$ & $\begin{array}{r}\sigma \\
(8)\end{array}$ & $\begin{array}{r}\Delta \sigma \\
(9)\end{array}$ & $\begin{array}{r}V_{\text {rot }} \\
(10)\end{array}$ & $\begin{array}{r}\Delta V_{\text {rot }} \\
(11)\end{array}$ \\
\hline 0 & 0.00 & 0.00 & 77.6 & 2.0 & 96.9 & 2.5 & 7.8 & 0.2 & 0.0 & 4.1 \\
12 & 0.65 & 0.05 & 75.4 & 2.0 & 94.2 & 2.5 & 7.5 & 0.2 & 42.1 & 20.0 \\
24 & 1.31 & 0.09 & 62.3 & 2.0 & 77.8 & 2.5 & 6.2 & 0.2 & 46.7 & 4.7 \\
36 & 1.96 & 0.14 & 55.1 & 2.0 & 68.9 & 2.5 & 5.5 & 0.2 & 64.0 & 6.4 \\
48 & 2.61 & 0.19 & 58.1 & 2.0 & 72.5 & 2.5 & 5.8 & 0.2 & 62.9 & 6.3 \\
60 & 3.26 & 0.23 & 61.0 & 2.0 & 76.2 & 2.5 & 6.1 & 0.2 & 73.4 & 4.1 \\
72 & 3.92 & 0.28 & 63.5 & 2.0 & 79.3 & 2.5 & 6.4 & 0.2 & 81.0 & 4.1 \\
84 & 4.57 & 0.33 & 66.1 & 2.0 & 82.5 & 2.5 & 6.6 & 0.2 & 87.9 & 4.1 \\
96 & 5.22 & 0.37 & 64.3 & 2.0 & 80.3 & 2.5 & 6.4 & 0.2 & 94.2 & 4.1 \\
108 & 5.87 & 0.42 & 58.4 & 2.0 & 73.0 & 2.5 & 5.8 & 0.2 & 102.3 & 4.1 \\
120 & 6.53 & 0.47 & 52.3 & 2.0 & 65.3 & 2.5 & 5.2 & 0.2 & 102.7 & 4.1 \\
132 & 7.18 & 0.51 & 47.5 & 2.0 & 59.3 & 2.5 & 4.8 & 0.2 & 104.2 & 4.1 \\
144 & 7.83 & 0.56 & 42.8 & 2.0 & 53.5 & 2.5 & 4.3 & 0.2 & 104.6 & 4.1 \\
156 & 8.49 & 0.61 & 43.3 & 2.0 & 54.0 & 2.5 & 4.3 & 0.2 & 104.0 & 4.1 \\
168 & 9.14 & 0.65 & 48.9 & 2.0 & 61.1 & 2.5 & 4.9 & 0.2 & 102.5 & 4.1 \\
180 & 9.79 & 0.70 & 54.7 & 2.0 & 68.3 & 2.5 & 5.5 & 0.2 & 100.9 & 4.1 \\
192 & 10.44 & 0.74 & 53.6 & 2.0 & 67.0 & 2.5 & 5.4 & 0.2 & 100.9 & 4.1 \\
210 & 11.42 & 0.81 & 51.6 & 2.0 & 64.4 & 2.5 & 5.2 & 0.2 & 101.0 & 8.2 \\
240 & 13.06 & 0.93 & 19.9 & 9.8 & 24.8 & 12.2 & 2.0 & 1.0 & 106.3 & 8.2 \\
270 & 14.69 & 1.05 & 24.1 & 3.1 & 30.1 & 3.8 & 2.4 & 0.3 & 96.7 & 8.2 \\
300 & 16.32 & 1.16 & 17.9 & 3.2 & 22.3 & 4.0 & 1.8 & 0.3 & 102.4 & 14.8 \\
330 & 17.95 & 1.28 & 9.9 & 5.2 & 12.4 & 6.5 & 1.0 & 0.5 & 109.7 & 23.8 \\
360 & 19.58 & 1.40 & 7.1 & 2.4 & 8.9 & 3.0 & 0.7 & 0.2 & 77.7 & 18.4 \\
390 & 21.21 & 1.51 & 6.0 & 3.8 & 7.5 & 4.7 & 0.6 & 0.4 & 128.3 & 24.2 \\
420 & 22.85 & 1.63 & 3.9 & 2.6 & 4.9 & 3.3 & 0.4 & 0.3 & 81.3 & 25.0 \\
450 & 24.48 & 1.75 & 1.3 & 2.0 & 1.6 & 2.5 & 0.1 & 0.2 & 119.7 & 12.0 \\
520 & 28.29 & 2.02 & 0.6 & 2.0 & 0.7 & 2.5 & 0.1 & 0.2 & 117.2 & 29.5 \\
590 & 32.09 & 2.29 & 0.6 & 2.0 & 0.8 & 2.5 & 0.1 & 0.2 & 127.2 & 25.0 \\
660 & 35.90 & 2.56 & 0.1 & 2.0 & 0.2 & 2.5 & 0.0 & 0.2 & 102.0 & 25.0 \\
\hline & & & & & & & & & &
\end{tabular}


G. I. G. Józsa: Kinematic modelling of disk galaxies. II., Online Material p 13

Table 6. continued. NGC 2541. (12) Inclination $\left({ }^{\circ}\right)$. (13) Error of $i\left({ }^{\circ}\right)$. (14) Position angle $\left({ }^{\circ}\right)$. (15) Error of PA $\left({ }^{\circ}\right)$. (16)-(23) are Cartesian components of the spin normal vector $\boldsymbol{n}$ of the ring and their errors. (16) Spin normal vector component towards W (natural units). (17) Error of $n_{\mathrm{W}}$ (natural units). (18) Spin normal vector component towards N (natural units). (19) Error of $n_{\mathrm{N}}$ (natural units). (20) Spin normal vector component towards observer (natural units). (21) Error of $n_{\mathrm{LOS}}$ (natural units). (22)-(25) contain the tip angle and the LON. The reference plane is chosen such that a reference ring with the inclination of $66^{\circ} 3$ and the position angle of 261.2 is seen face-on. (22) Tip (warp) angle $\left({ }^{\circ}\right)$. (23) Error of tip $\left({ }^{\circ}\right)$. (24) $\operatorname{LON}\left({ }^{\circ}\right) .(25)$ Error of $\operatorname{LON}\left({ }^{\circ}\right)$.

\begin{tabular}{|c|c|c|c|c|c|c|c|c|c|c|c|c|c|c|}
\hline $\begin{array}{r}r_{\mathrm{p}} \\
(1)\end{array}$ & $\begin{array}{r}i \\
(12)\end{array}$ & $\begin{array}{r}\Delta i \\
(13)\end{array}$ & $\begin{array}{r}\text { PA } \\
(14)\end{array}$ & $\begin{array}{r}\Delta \mathrm{PA} \\
(15)\end{array}$ & $\begin{array}{r}n_{\mathrm{W}} \\
(16)\end{array}$ & $\begin{array}{r}\Delta n_{\mathrm{W}} \\
(17)\end{array}$ & $\begin{array}{r}n_{\mathrm{N}} \\
(18)\end{array}$ & $\begin{array}{l}\Delta n_{\mathrm{N}} \\
(19)\end{array}$ & $\begin{array}{r}n_{\mathrm{LOS}} \\
(20)\end{array}$ & $\begin{array}{r}\Delta n_{\mathrm{LOS}} \\
\quad(21)\end{array}$ & $\begin{array}{r}t i p \\
(22)\end{array}$ & $\begin{array}{r}\Delta t i p \\
(23)\end{array}$ & $\begin{array}{r}L O N \\
(24)\end{array}$ & $\begin{array}{r}\Delta L O N \\
(25)\end{array}$ \\
\hline 0 & 83.3 & 10.0 & 283.2 & 15.0 & -0.967 & 0.063 & -0.227 & 0.253 & 0.117 & 0.173 & 27.2 & 13.1 & -125 & 28 \\
\hline 12 & 83.3 & 10.0 & 283.2 & 15.0 & -0.967 & 0.063 & -0.227 & 0.253 & 0.117 & 0.173 & 27.2 & 13.1 & -125 & 28 \\
\hline 24 & 77.8 & 10.0 & 271.7 & 15.0 & -0.977 & 0.038 & -0.029 & 0.256 & 0.211 & 0.171 & 15.3 & 12.2 & -137 & 50 \\
\hline 36 & 65.6 & 10.0 & 263.1 & 15.0 & -0.904 & 0.077 & 0.109 & 0.237 & 0.414 & 0.159 & 1.9 & 13.2 & -67 & 180 \\
\hline 48 & 66.3 & 10.0 & 261.2 & 15.0 & -0.905 & 0.078 & 0.141 & 0.237 & 0.402 & 0.160 & 0.0 & 0.0 & -125 & 180 \\
\hline 60 & 67.0 & 5.3 & 259.2 & 7.9 & -0.905 & 0.043 & 0.172 & 0.126 & 0.390 & 0.086 & 1.9 & 7.2 & 112 & 170 \\
\hline 72 & 66.8 & 4.0 & 259.4 & 6.0 & -0.904 & 0.032 & 0.169 & 0.095 & 0.393 & 0.064 & 1.7 & 5.5 & 107 & 140 \\
\hline 84 & 66.6 & 4.0 & 259.5 & 4.0 & -0.903 & 0.030 & 0.167 & 0.064 & 0.397 & 0.064 & 1.5 & 3.8 & 101 & 149 \\
\hline 96 & 66.0 & 4.0 & 259.8 & 4.0 & -0.899 & 0.030 & 0.163 & 0.063 & 0.407 & 0.064 & 1.3 & 3.7 & 77 & 172 \\
\hline 108 & 65.0 & 4.0 & 260.1 & 4.0 & -0.893 & 0.031 & 0.156 & 0.063 & 0.423 & 0.063 & 1.7 & 3.9 & 37 & 130 \\
\hline 120 & 64.0 & 5.2 & 260.4 & 4.0 & -0.886 & 0.041 & 0.150 & 0.062 & 0.439 & 0.081 & 2.5 & 5.1 & 17 & 88 \\
\hline 132 & 63.9 & 5.0 & 260.0 & 4.0 & -0.884 & 0.040 & 0.156 & 0.062 & 0.440 & 0.079 & 2.6 & 4.9 & 23 & 85 \\
\hline 144 & 63.9 & 4.9 & 259.7 & 5.5 & -0.883 & 0.040 & 0.161 & 0.084 & 0.440 & 0.077 & 2.8 & 5.0 & 29 & 101 \\
\hline 156 & 64.0 & 4.0 & 259.1 & 4.6 & -0.882 & 0.033 & 0.170 & 0.071 & 0.438 & 0.063 & 3.0 & 4.1 & 39 & 78 \\
\hline 168 & 64.3 & 4.0 & 258.2 & 4.0 & -0.882 & 0.032 & 0.184 & 0.062 & 0.434 & 0.063 & 3.3 & 3.8 & 52 & 67 \\
\hline 180 & 64.6 & 4.0 & 257.4 & 4.0 & -0.882 & 0.032 & 0.197 & 0.062 & 0.429 & 0.063 & 3.8 & 3.7 & 63 & 60 \\
\hline 192 & 63.9 & 4.0 & 257.1 & 4.0 & -0.875 & 0.033 & 0.201 & 0.061 & 0.440 & 0.063 & 4.5 & 3.8 & 56 & 51 \\
\hline 210 & 62.8 & 4.6 & 256.5 & 4.0 & -0.865 & 0.039 & 0.207 & 0.061 & 0.457 & 0.072 & 5.5 & 4.1 & 49 & 45 \\
\hline 240 & 58.4 & 4.0 & 261.5 & 4.0 & -0.843 & 0.037 & 0.126 & 0.059 & 0.524 & 0.059 & 7.9 & 4.0 & -2 & 25 \\
\hline 270 & 58.1 & 4.0 & 271.6 & 4.0 & -0.849 & 0.037 & -0.024 & 0.059 & 0.528 & 0.059 & 12.3 & 3.7 & -46 & 18 \\
\hline 300 & 56.0 & 4.0 & 271.9 & 4.0 & -0.828 & 0.039 & -0.028 & 0.058 & 0.560 & 0.058 & 14.0 & 3.7 & -40 & 16 \\
\hline 330 & 58.4 & 5.0 & 277.2 & 5.7 & -0.845 & 0.047 & -0.107 & 0.085 & 0.524 & 0.074 & 16.2 & 4.9 & -57 & 18 \\
\hline 360 & 58.4 & 4.0 & 274.9 & 5.3 & -0.849 & 0.037 & -0.073 & 0.079 & 0.524 & 0.059 & 14.5 & 4.5 & -54 & 17 \\
\hline 390 & 62.8 & 6.3 & 280.4 & 10.0 & -0.875 & 0.057 & -0.161 & 0.153 & 0.456 & 0.097 & 17.7 & 8.9 & -75 & 21 \\
\hline 420 & 59.8 & 5.9 & 275.0 & 10.0 & -0.861 & 0.053 & -0.075 & 0.150 & 0.503 & 0.088 & 13.9 & 8.3 & -59 & 27 \\
\hline 450 & 62.6 & 15.0 & 282.9 & 5.3 & -0.865 & 0.119 & -0.198 & 0.084 & 0.461 & 0.232 & 19.9 & 4.9 & -75 & 44 \\
\hline 520 & 65.1 & 7.5 & 277.8 & 5.5 & -0.899 & 0.056 & -0.124 & 0.087 & 0.421 & 0.118 & 15.2 & 5.1 & -82 & 29 \\
\hline 590 & 68.4 & 6.3 & 287.6 & 6.6 & -0.886 & 0.050 & -0.281 & 0.102 & 0.368 & 0.103 & 24.5 & 6.2 & -90 & 16 \\
\hline 660 & 58.6 & 9.7 & 277.9 & 6.0 & -0.845 & 0.088 & -0.117 & 0.090 & 0.521 & 0.144 & 16.7 & 6.1 & -59 & 32 \\
\hline
\end{tabular}


G. I. G. Józsa: Kinematic modelling of disk galaxies. II., Online Material p 14

Table 6. continued. UGC 3580.

\begin{tabular}{rrrrrrrrrrr}
\hline \hline$r_{\mathrm{p}}$ & $r_{\mathrm{t}}$ & $\Delta r_{\mathrm{t}}$ & $I_{\text {tot,f }}$ & $\Delta I_{\text {tot,f }}$ & $N_{\mathrm{HI}}$ & $\Delta N_{\mathrm{HI}}$ & $\sigma$ & $\Delta \sigma$ & $V_{\text {rot }}$ & $\Delta V_{\text {rot }}$ \\
$(1)$ & $(2)$ & $(3)$ & $(4)$ & $(5)$ & $(6)$ & $(7)$ & $(8)$ & $(9)$ & $(10)$ & $(11)$ \\
\hline 0 & 0.00 & 0.00 & 75.9 & 35.2 & 94.8 & 44.0 & 7.6 & 3.5 & 0.0 & 0.0 \\
12 & 0.88 & 0.20 & 94.8 & 45.9 & 118.4 & 57.3 & 9.5 & 4.6 & 78.5 & 7.9 \\
24 & 1.76 & 0.40 & 81.6 & 15.9 & 101.9 & 19.9 & 8.2 & 1.6 & 77.6 & 7.8 \\
36 & 2.64 & 0.61 & 55.0 & 12.3 & 68.6 & 15.3 & 5.5 & 1.2 & 94.3 & 9.4 \\
48 & 3.51 & 0.81 & 34.9 & 5.4 & 43.6 & 6.7 & 3.5 & 0.5 & 100.8 & 9.8 \\
60 & 4.39 & 1.01 & 42.0 & 5.0 & 52.4 & 6.3 & 4.2 & 0.5 & 95.0 & 7.6 \\
72 & 5.27 & 1.21 & 42.7 & 2.1 & 53.3 & 2.7 & 4.3 & 0.2 & 103.7 & 4.1 \\
84 & 6.15 & 1.42 & 41.8 & 10.1 & 52.2 & 12.6 & 4.2 & 1.0 & 110.7 & 4.1 \\
120 & 8.78 & 2.02 & 35.9 & 2.5 & 44.8 & 3.1 & 3.6 & 0.3 & 106.2 & 4.1 \\
150 & 10.98 & 2.53 & 12.9 & 5.4 & 16.1 & 6.7 & 1.3 & 0.5 & 114.4 & 8.2 \\
180 & 13.18 & 3.03 & 17.4 & 2.0 & 21.7 & 2.5 & 1.7 & 0.2 & 121.1 & 8.2 \\
210 & 15.37 & 3.54 & 7.6 & 2.4 & 9.5 & 3.0 & 0.8 & 0.2 & 122.2 & 8.7 \\
240 & 17.57 & 4.04 & 2.8 & 2.2 & 3.5 & 2.7 & 0.3 & 0.2 & 135.3 & 10.0 \\
270 & 19.76 & 4.55 & 4.5 & 2.0 & 5.7 & 2.5 & 0.5 & 0.2 & 111.2 & 8.2 \\
300 & 21.96 & 5.05 & 1.4 & 2.0 & 1.7 & 2.5 & 0.1 & 0.2 & 120.3 & 8.2 \\
330 & 24.16 & 5.56 & 1.7 & 2.0 & 2.1 & 2.5 & 0.2 & 0.2 & 113.2 & 10.0 \\
375 & 27.45 & 6.32 & 0.8 & 2.0 & 1.0 & 2.5 & 0.1 & 0.2 & 121.3 & 16.9 \\
420 & 30.74 & 7.08 & 0.7 & 2.0 & 0.9 & 2.5 & 0.1 & 0.2 & 89.6 & 27.0 \\
\hline
\end{tabular}

Table 6. continued. UGC 3580. The reference ring for (22)-(25) has an inclination of $67^{\circ} .2$ and a position angle of $96^{\circ} .3$.

\begin{tabular}{|c|c|c|c|c|c|c|c|c|c|c|c|c|c|c|}
\hline $\begin{array}{r}r_{\mathrm{p}} \\
(1)\end{array}$ & $\begin{array}{r}i \\
(12)\end{array}$ & $\begin{array}{r}\Delta i \\
(13)\end{array}$ & $\begin{array}{r}\mathrm{PA} \\
(14)\end{array}$ & $\begin{array}{r}\Delta \mathrm{PA} \\
(15)\end{array}$ & $\begin{array}{r}n_{\mathrm{W}} \\
(16)\end{array}$ & $\begin{array}{r}\Delta n_{\mathrm{W}} \\
(17)\end{array}$ & $\begin{array}{r}n_{\mathrm{N}} \\
(18)\end{array}$ & $\begin{array}{l}\Delta n_{\mathrm{N}} \\
(19)\end{array}$ & $\begin{array}{r}n_{\mathrm{LOS}} \\
(20)\end{array}$ & $\begin{array}{r}\Delta n_{\mathrm{LOS}} \\
(21)\end{array}$ & $\begin{array}{r}t i p \\
(22)\end{array}$ & $\begin{array}{r}\Delta \text { tip } \\
(23)\end{array}$ & $\begin{array}{r}\text { LON } \\
(24)\end{array}$ & $\begin{array}{r}L O N \\
(25)\end{array}$ \\
\hline 0 & 67.2 & 8.6 & 96.3 & 8.6 & 0.916 & 0.060 & 0.102 & 0.138 & 0.388 & 0.139 & 0.0 & 0.0 & 123 & 180 \\
\hline 12 & 67.2 & 8.6 & 96.3 & 8.6 & 0.916 & 0.060 & 0.102 & 0.138 & 0.388 & 0.139 & 0.0 & 0.0 & 123 & 180 \\
\hline 24 & 67.2 & 8.4 & 96.3 & 4.0 & 0.916 & 0.057 & 0.102 & 0.064 & 0.388 & 0.135 & 0.0 & 0.0 & 123 & 180 \\
\hline 36 & 67.2 & 6.7 & 96.3 & 4.0 & 0.916 & 0.046 & 0.102 & 0.064 & 0.388 & 0.108 & 0.0 & 0.0 & 123 & 180 \\
\hline 48 & 67.2 & 4.0 & 96.3 & 4.0 & 0.916 & 0.028 & 0.102 & 0.064 & 0.388 & 0.064 & 0.0 & 0.0 & 123 & 180 \\
\hline 60 & 67.2 & 4.0 & 96.3 & 4.0 & 0.916 & 0.028 & 0.102 & 0.064 & 0.388 & 0.064 & 0.0 & 0.0 & 123 & 180 \\
\hline 72 & 67.2 & 4.0 & 96.3 & 4.0 & 0.916 & 0.028 & 0.102 & 0.064 & 0.388 & 0.064 & 0.0 & 0.0 & 123 & 180 \\
\hline 84 & 67.2 & 4.0 & 96.3 & 4.0 & 0.916 & 0.028 & 0.102 & 0.064 & 0.388 & 0.064 & 0.0 & 0.0 & 123 & 180 \\
\hline 120 & 67.2 & 4.0 & 96.3 & 4.0 & 0.916 & 0.028 & 0.102 & 0.064 & 0.388 & 0.064 & 0.0 & 0.0 & 123 & 180 \\
\hline 150 & 67.2 & 5.6 & 96.3 & 4.0 & 0.916 & 0.038 & 0.102 & 0.064 & 0.388 & 0.090 & 0.0 & 0.0 & 123 & 180 \\
\hline 180 & 70.1 & 4.0 & 91.3 & 4.0 & 0.940 & 0.024 & 0.021 & 0.066 & 0.340 & 0.066 & 5.5 & 3.9 & 121 & 41 \\
\hline 210 & 67.6 & 4.1 & 87.4 & 4.0 & 0.923 & 0.028 & -0.042 & 0.064 & 0.382 & 0.067 & 8.2 & 3.7 & 91 & 29 \\
\hline 240 & 66.8 & 8.9 & 81.3 & 10.0 & 0.909 & 0.065 & -0.139 & 0.159 & 0.394 & 0.142 & 13.9 & 9.2 & 86 & 37 \\
\hline 270 & 74.9 & 4.0 & 82.1 & 4.1 & 0.956 & 0.020 & -0.133 & 0.069 & 0.261 & 0.067 & 15.5 & 4.0 & 117 & 15 \\
\hline 300 & 68.4 & 10.9 & 80.4 & 4.6 & 0.916 & 0.070 & -0.156 & 0.074 & 0.369 & 0.177 & 14.8 & 4.5 & 91 & 42 \\
\hline 330 & 70.4 & 4.0 & 82.5 & 4.0 & 0.934 & 0.025 & -0.123 & 0.065 & 0.336 & 0.066 & 13.3 & 3.8 & 101 & 17 \\
\hline 375 & 67.1 & 4.0 & 79.2 & 4.4 & 0.905 & 0.030 & -0.173 & 0.070 & 0.389 & 0.064 & 15.8 & 4.1 & 86 & 15 \\
\hline 420 & 59.0 & 4.6 & 78.1 & 9.5 & 0.839 & 0.050 & -0.177 & 0.139 & 0.515 & 0.068 & 18.2 & 7.8 & 59 & 17 \\
\hline
\end{tabular}


Table 6. continued. NGC 5204.

\begin{tabular}{rrrrrrrrrrr}
\hline \hline$r_{\mathrm{p}}$ & $r_{\mathrm{t}}$ & $\Delta r_{\mathrm{t}}$ & $I_{\text {tot,f }}$ & $\Delta I_{\text {tot,f }}$ & $N_{\mathrm{H}_{\mathrm{I}}}$ & $\Delta N_{\mathrm{H} \text { I }}$ & $\sigma$ & $\Delta \sigma$ & $V_{\text {rot }}$ & $\Delta V_{\text {rot }}$ \\
$(1)$ & $(2)$ & $(3)$ & $(4)$ & $(5)$ & $(6)$ & $(7)$ & $(8)$ & $(9)$ & $(10)$ & $(11)$ \\
\hline 0 & 0.00 & 0.00 & 184.7 & 17.4 & 230.6 & 21.7 & 18.5 & 1.7 & 0.0 & 4.1 \\
12 & 0.24 & 0.06 & 111.8 & 59.4 & 139.6 & 74.2 & 11.2 & 5.9 & 47.7 & 14.4 \\
24 & 0.48 & 0.11 & 95.3 & 29.9 & 119.0 & 37.3 & 9.5 & 3.0 & 47.5 & 4.1 \\
36 & 0.72 & 0.17 & 73.2 & 17.3 & 91.4 & 21.6 & 7.3 & 1.7 & 53.8 & 4.1 \\
48 & 0.96 & 0.23 & 69.8 & 16.5 & 87.1 & 20.6 & 7.0 & 1.7 & 59.9 & 4.1 \\
60 & 1.20 & 0.28 & 57.4 & 22.1 & 71.7 & 27.5 & 5.7 & 2.2 & 65.4 & 4.1 \\
72 & 1.45 & 0.34 & 52.7 & 2.0 & 65.9 & 2.5 & 5.3 & 0.2 & 64.6 & 4.1 \\
84 & 1.69 & 0.40 & 52.0 & 12.6 & 65.0 & 15.7 & 5.2 & 1.3 & 64.5 & 5.1 \\
96 & 1.93 & 0.45 & 54.8 & 20.4 & 68.4 & 25.5 & 5.5 & 2.0 & 68.9 & 4.4 \\
108 & 2.17 & 0.51 & 51.0 & 20.4 & 63.6 & 25.4 & 5.1 & 2.0 & 71.2 & 4.1 \\
120 & 2.41 & 0.57 & 38.8 & 5.4 & 48.5 & 6.7 & 3.9 & 0.5 & 70.1 & 4.1 \\
132 & 2.65 & 0.62 & 43.4 & 3.1 & 54.2 & 3.9 & 4.3 & 0.3 & 69.8 & 4.1 \\
144 & 2.89 & 0.68 & 40.1 & 3.9 & 50.1 & 4.8 & 4.0 & 0.4 & 66.2 & 4.1 \\
156 & 3.13 & 0.74 & 53.0 & 12.2 & 66.2 & 15.2 & 5.3 & 1.2 & 64.2 & 4.1 \\
168 & 3.37 & 0.79 & 49.9 & 9.5 & 62.3 & 11.9 & 5.0 & 1.0 & 58.7 & 4.1 \\
180 & 3.61 & 0.85 & 41.7 & 2.0 & 52.1 & 2.5 & 4.2 & 0.2 & 56.3 & 8.2 \\
210 & 4.22 & 0.99 & 21.7 & 2.0 & 27.0 & 2.5 & 2.2 & 0.2 & 72.2 & 11.4 \\
240 & 4.82 & 1.13 & 11.5 & 2.6 & 14.4 & 3.2 & 1.2 & 0.3 & 80.4 & 8.9 \\
270 & 5.42 & 1.27 & 11.9 & 2.0 & 14.9 & 2.5 & 1.2 & 0.2 & 79.6 & 9.7 \\
300 & 6.02 & 1.41 & 11.2 & 2.1 & 14.0 & 2.6 & 1.1 & 0.2 & 84.0 & 8.2 \\
330 & 6.63 & 1.56 & 9.1 & 3.0 & 11.3 & 3.7 & 0.9 & 0.3 & 82.9 & 8.2 \\
360 & 7.23 & 1.70 & 10.2 & 2.0 & 12.8 & 2.5 & 1.0 & 0.2 & 81.1 & 8.2 \\
390 & 7.83 & 1.84 & 7.8 & 2.0 & 9.7 & 2.5 & 0.8 & 0.2 & 84.8 & 8.2 \\
420 & 8.43 & 1.98 & 4.2 & 2.0 & 5.2 & 2.5 & 0.4 & 0.2 & 105.0 & 25.0 \\
450 & 9.04 & 2.12 & 4.7 & 2.0 & 5.9 & 2.5 & 0.5 & 0.2 & 131.2 & 25.0 \\
\hline & & & & & & & & & &
\end{tabular}

Table 6. continued. NGC 5204. The reference ring for (22)-(25) has an inclination of 56.1 and a position angle of 257.4.

\begin{tabular}{|c|c|c|c|c|c|c|c|c|c|c|c|c|c|c|}
\hline $\begin{array}{r}r_{\mathrm{p}} \\
(1)\end{array}$ & $\begin{array}{r}i \\
(12)\end{array}$ & $\begin{array}{r}\Delta i \\
(13)\end{array}$ & $\begin{array}{r}\text { PA } \\
(14)\end{array}$ & $\begin{array}{r}\Delta \mathrm{PA} \\
(15)\end{array}$ & $\begin{array}{r}n_{\mathrm{W}} \\
(16)\end{array}$ & $\begin{array}{r}\Delta n_{\mathrm{W}} \\
\text { (17) }\end{array}$ & $\begin{array}{r}n_{\mathrm{N}} \\
(18)\end{array}$ & $\begin{array}{l}\Delta n_{\mathrm{N}} \\
(19)\end{array}$ & $\begin{array}{l}n_{\mathrm{LOS}} \\
(20)\end{array}$ & $\begin{array}{r}\Delta n_{\mathrm{LOS}} \\
(21)\end{array}$ & $\begin{array}{r}t i p \\
(22)\end{array}$ & $\begin{array}{r}\Delta t i p \\
(23)\end{array}$ & $\begin{array}{r}L O N \\
(24)\end{array}$ & $\begin{array}{r}L O N \\
(25)\end{array}$ \\
\hline 0 & 56.1 & 23.7 & 257.4 & 26.2 & -0.810 & 0.240 & 0.181 & 0.374 & 0.558 & 0.343 & 0.0 & 0.0 & 349.0 & 180.0 \\
\hline 12 & 56.1 & 14.1 & 257.4 & 38.7 & -0.810 & 0.181 & 0.181 & 0.547 & 0.558 & 0.204 & 0.0 & 0.0 & 349.0 & 180.0 \\
\hline 24 & 56.1 & 4.0 & 257.4 & 11.0 & -0.810 & 0.051 & 0.181 & 0.156 & 0.558 & 0.058 & 0.0 & 0.0 & 349.0 & 180.0 \\
\hline 36 & 56.1 & 7.4 & 257.4 & 6.2 & -0.810 & 0.073 & 0.181 & 0.089 & 0.558 & 0.107 & 0.0 & 0.0 & 349.0 & 180.0 \\
\hline 48 & 56.1 & 9.5 & 257.4 & 4.0 & -0.810 & 0.092 & 0.181 & 0.060 & 0.558 & 0.138 & 0.0 & 0.0 & 349.0 & 180.0 \\
\hline 60 & 56.1 & 11.7 & 257.4 & 7.2 & -0.810 & 0.113 & 0.181 & 0.105 & 0.558 & 0.169 & 0.0 & 0.0 & 349.0 & 180.0 \\
\hline 72 & 56.1 & 6.2 & 257.4 & 4.3 & -0.810 & 0.061 & 0.181 & 0.062 & 0.558 & 0.090 & 0.0 & 0.0 & 349.0 & 180.0 \\
\hline 84 & 56.1 & 4.0 & 257.4 & 4.0 & -0.810 & 0.040 & 0.181 & 0.057 & 0.558 & 0.058 & 0.0 & 0.0 & 349.0 & 180.0 \\
\hline 96 & 56.1 & 4.0 & 257.4 & 4.0 & -0.810 & 0.040 & 0.181 & 0.057 & 0.558 & 0.058 & 0.0 & 0.0 & 349.0 & 180.0 \\
\hline 108 & 56.1 & 4.0 & 257.4 & 4.0 & -0.810 & 0.040 & 0.181 & 0.057 & 0.558 & 0.058 & 0.0 & 0.0 & 349.0 & 180.0 \\
\hline 120 & 56.1 & 4.0 & 257.4 & 4.0 & -0.810 & 0.040 & 0.181 & 0.057 & 0.558 & 0.058 & 0.0 & 0.0 & 349.0 & 180.0 \\
\hline 132 & 56.1 & 4.0 & 257.4 & 4.0 & -0.810 & 0.040 & 0.181 & 0.057 & 0.558 & 0.058 & 0.0 & 0.0 & 349.0 & 180.0 \\
\hline 144 & 56.1 & 4.0 & 257.4 & 4.0 & -0.810 & 0.040 & 0.181 & 0.057 & 0.558 & 0.058 & 0.0 & 0.0 & 349.0 & 180.0 \\
\hline 156 & 50.4 & 4.0 & 258.8 & 4.0 & -0.756 & 0.045 & 0.150 & 0.053 & 0.638 & 0.054 & 5.8 & 4.0 & 349.8 & 31.1 \\
\hline 168 & 48.0 & 4.0 & 261.4 & 4.0 & -0.735 & 0.047 & 0.111 & 0.052 & 0.669 & 0.052 & 8.6 & 3.9 & 339.9 & 21.1 \\
\hline 180 & 42.7 & 4.0 & 264.0 & 4.0 & -0.674 & 0.051 & 0.070 & 0.047 & 0.735 & 0.047 & 14.3 & 3.9 & 341.5 & 12.0 \\
\hline 210 & 28.9 & 4.0 & 286.3 & 6.1 & -0.464 & 0.060 & -0.136 & 0.053 & 0.876 & 0.034 & 32.9 & 3.5 & 334.6 & 6.6 \\
\hline 240 & 30.1 & 4.0 & 310.6 & 18.9 & -0.381 & 0.117 & -0.327 & 0.132 & 0.865 & 0.035 & 42.9 & 9.3 & 323.9 & 6.5 \\
\hline 270 & 34.8 & 4.0 & 309.7 & 4.0 & -0.439 & 0.051 & -0.365 & 0.048 & 0.821 & 0.040 & 41.6 & 2.4 & 317.1 & 6.0 \\
\hline 300 & 37.6 & 4.0 & 317.5 & 4.0 & -0.412 & 0.049 & -0.449 & 0.050 & 0.793 & 0.043 & 46.0 & 2.5 & 312.7 & 5.6 \\
\hline 330 & 39.3 & 4.0 & 316.1 & 4.0 & -0.439 & 0.049 & -0.456 & 0.050 & 0.774 & 0.044 & 45.2 & 2.6 & 310.3 & 5.7 \\
\hline 360 & 42.4 & 4.0 & 318.5 & 4.0 & -0.447 & 0.049 & -0.505 & 0.050 & 0.738 & 0.047 & 46.9 & 2.8 & 306.1 & 5.5 \\
\hline 390 & 39.0 & 4.0 & 319.4 & 4.0 & -0.409 & 0.049 & -0.478 & 0.050 & 0.778 & 0.044 & 47.2 & 2.6 & 310.9 & 5.5 \\
\hline 420 & 34.1 & 25.0 & 322.2 & 25.0 & -0.344 & 0.294 & -0.443 & 0.322 & 0.828 & 0.245 & 48.6 & 14.1 & 317.5 & 33.4 \\
\hline 450 & 23.3 & 25.0 & 322.1 & 25.0 & -0.242 & 0.281 & -0.312 & 0.334 & 0.919 & 0.172 & 49.2 & 10.4 & 331.9 & 32.8 \\
\hline
\end{tabular}


G. I. G. Józsa: Kinematic modelling of disk galaxies. II., Online Material p 16

1)

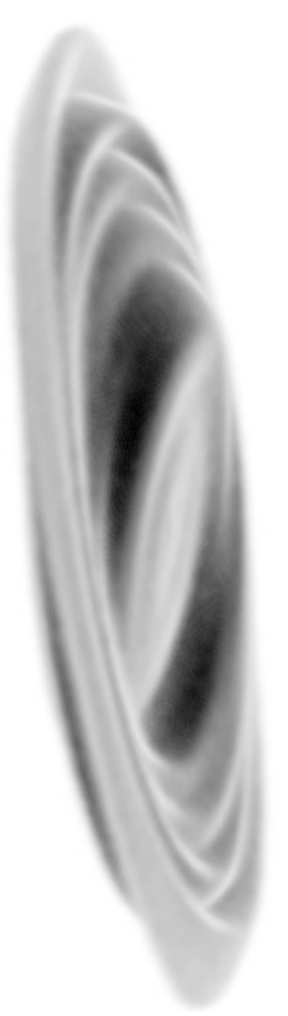

Fig. 9. Three-dimensional models of the H I disk of the galaxies according to the derived tilted-ring models. The models are rotated and artificially made opaque in order to again show the presence of an outer planar disk. 1) NGC 2541. 2) UGC 3580 3) NGC 5204. 
G. I. G. Józsa: Kinematic modelling of disk galaxies. II., Online Material p 17

1)

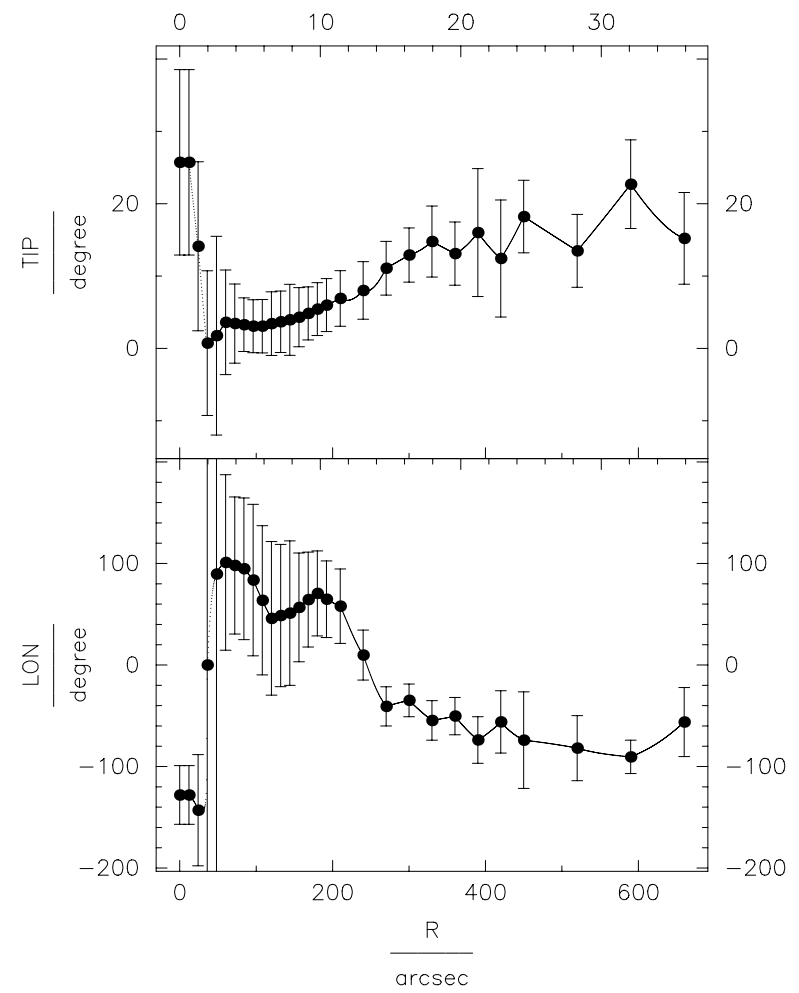

3)

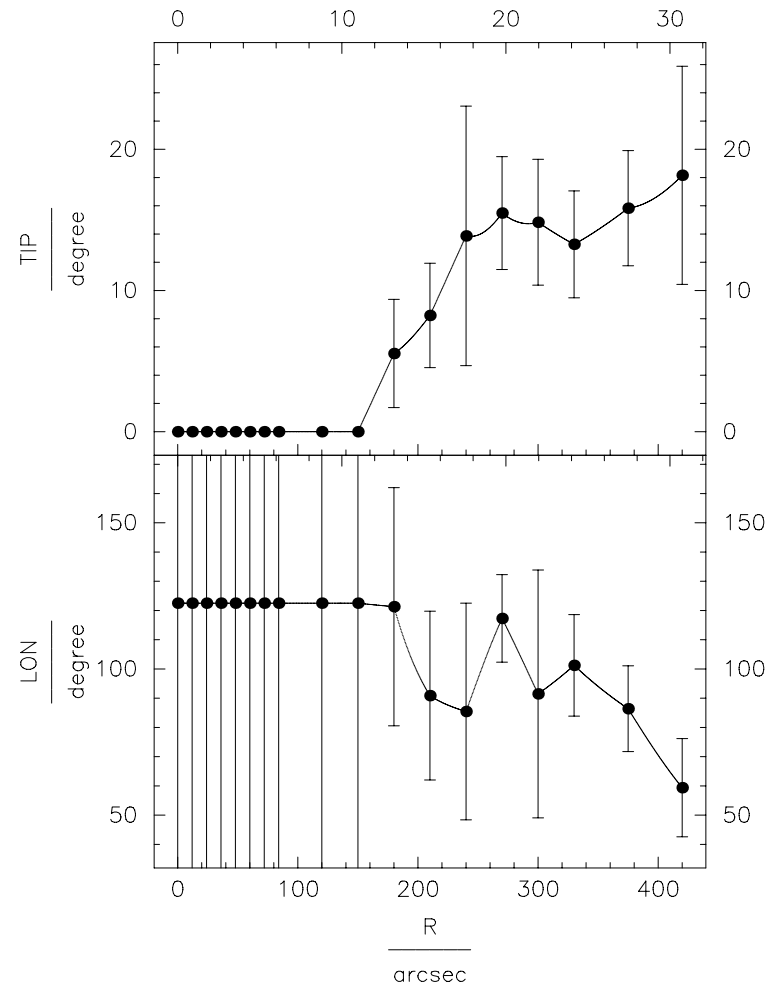

2)

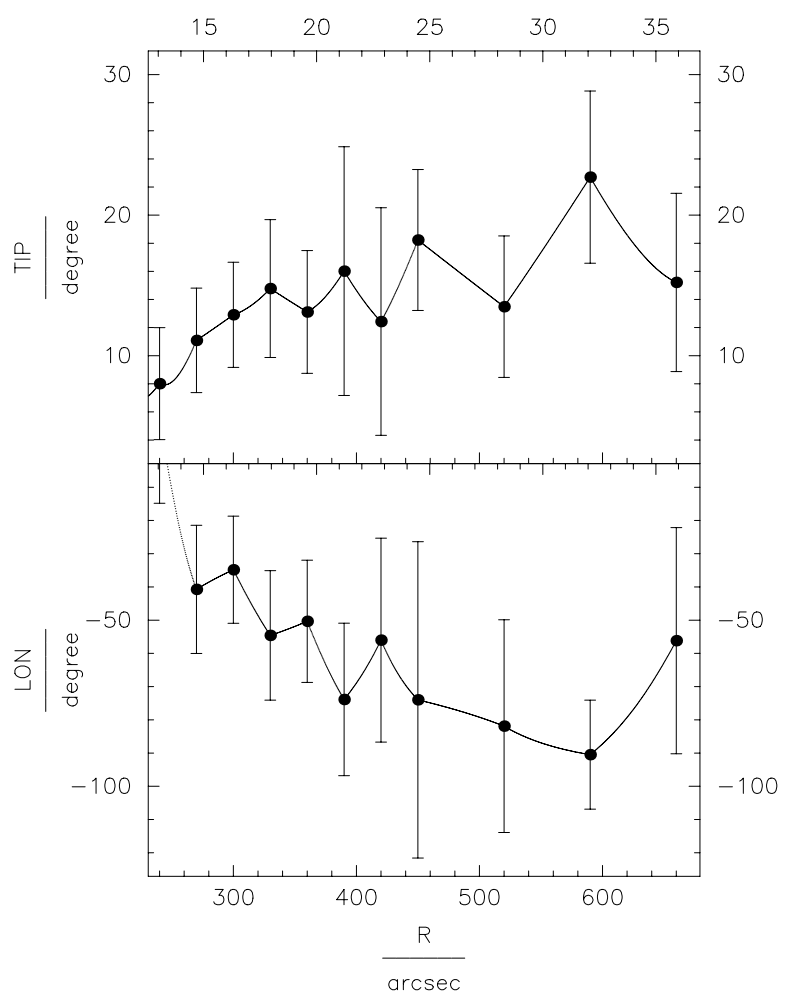

4)

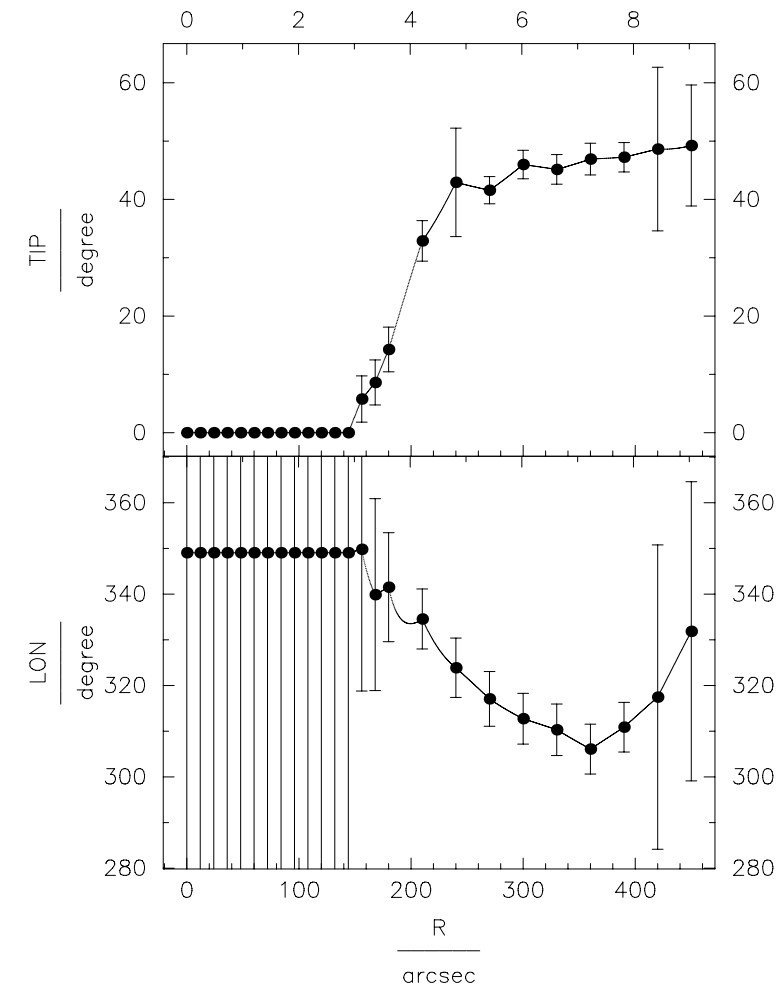

Fig. 10. Linear representation of tip angle (upper panels) and LON (lower panels) as given in the tip-LON diagrams in Fig. 4a). 1) NGC 2541. 2) NGC 2541, blow-up. 3) UGC 3580. 4) NGC 5204. 
G. I. G. Józsa: Kinematic modelling of disk galaxies. II., Online Material p 18

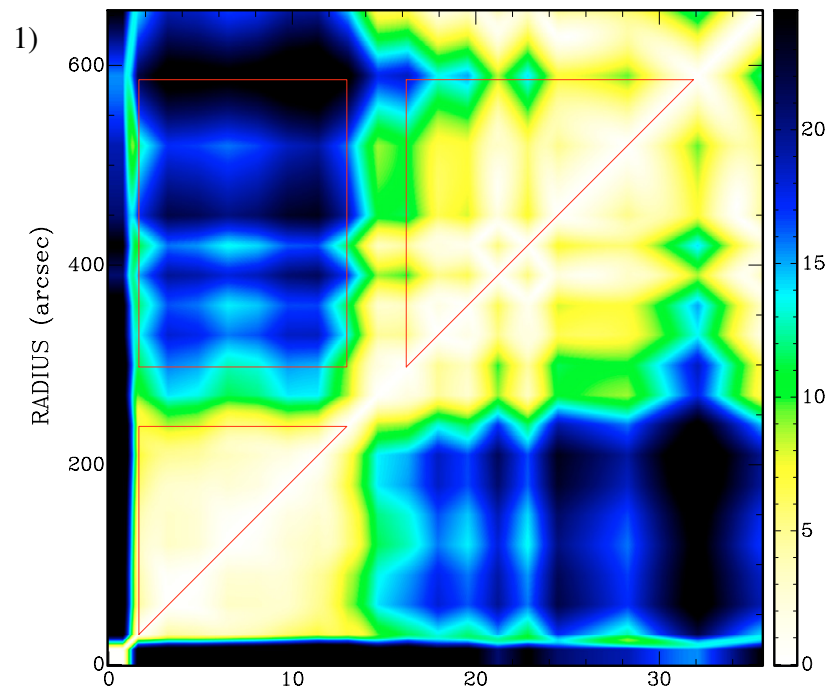

RADIUS (kpc)
2)

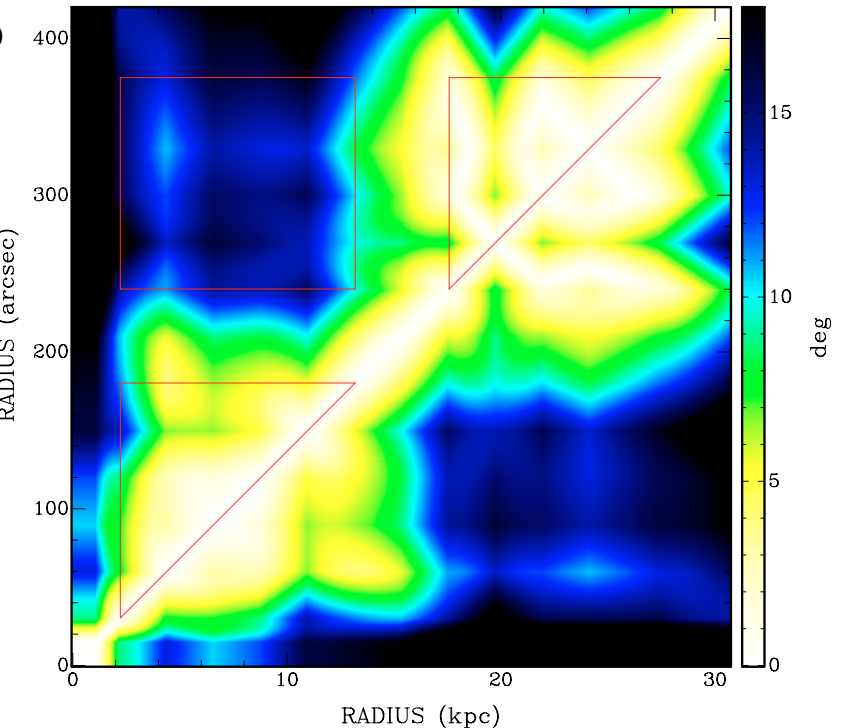

RADIUS (kpc)

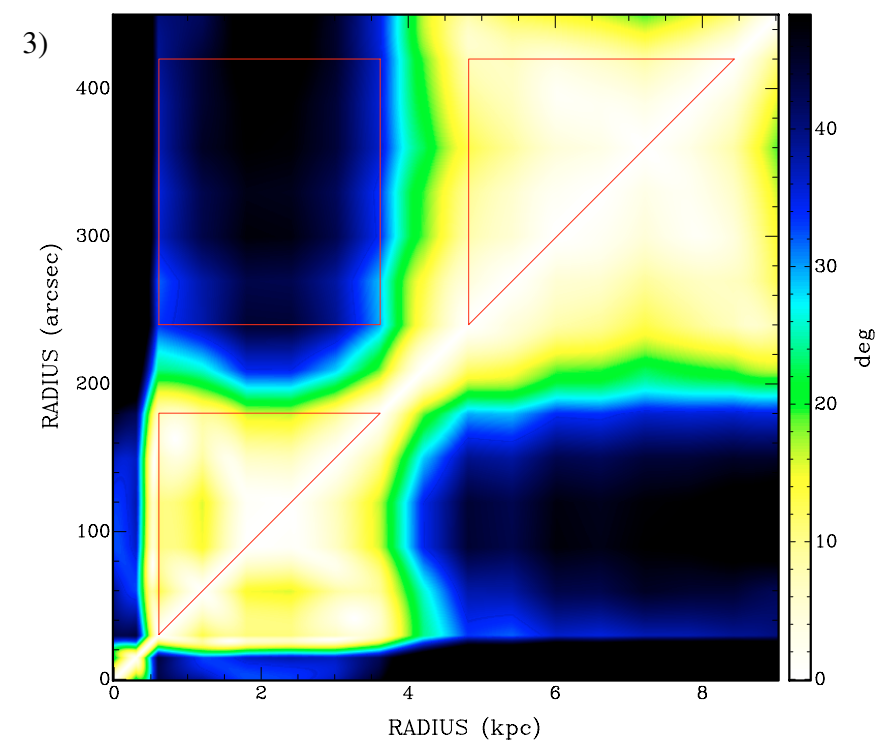

Fig. 11. Tiltogram of the galaxies, showing the mutual inclination $\theta_{\text {tilt }}$ of rings at different radii, derived from the best-fit parameters fitting to the low-resolution data cube without enforcing an inner flat disk. The mean mutual inclinations $\bar{\theta}_{\text {tilt }}^{\text {in/out/mut }}$ of Table 4 were determined inside the regions encompassed by the red lines. 1) NGC 2541. 2) UGC 3580. 3) NGC 5204. 


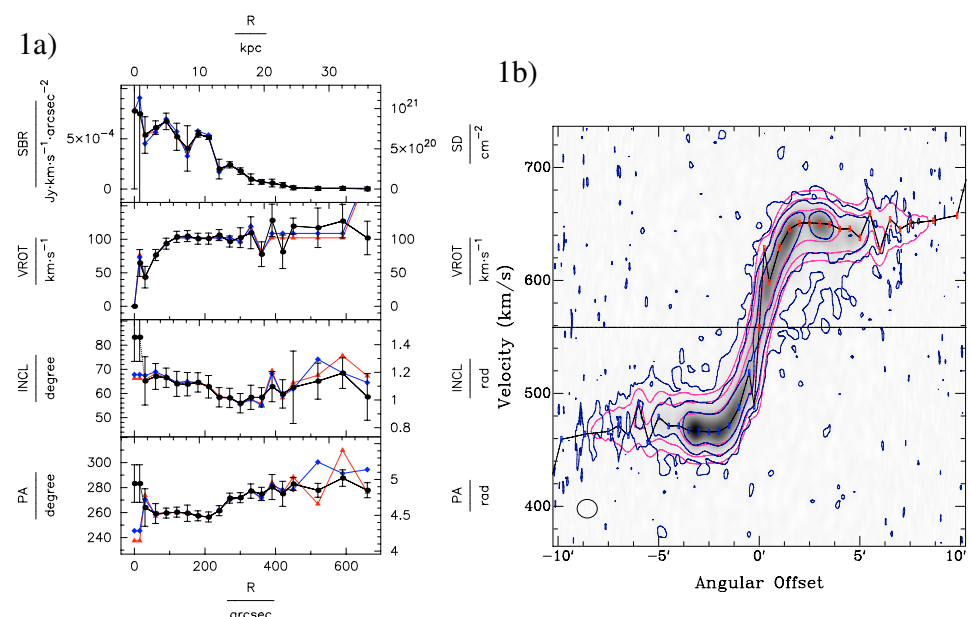

1c)

2a)

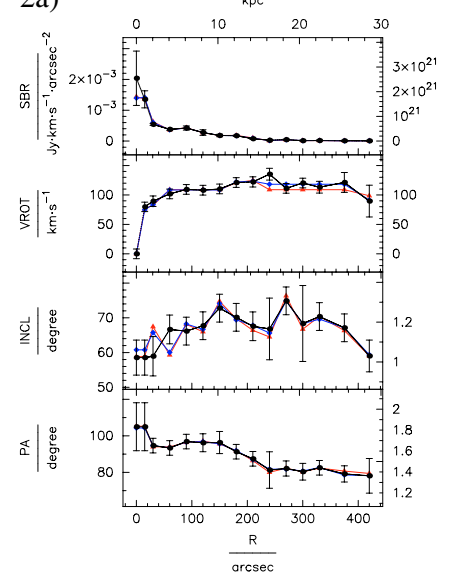

3a)

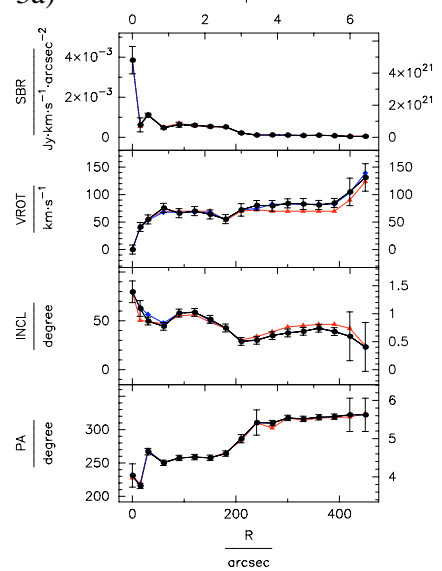

2b)

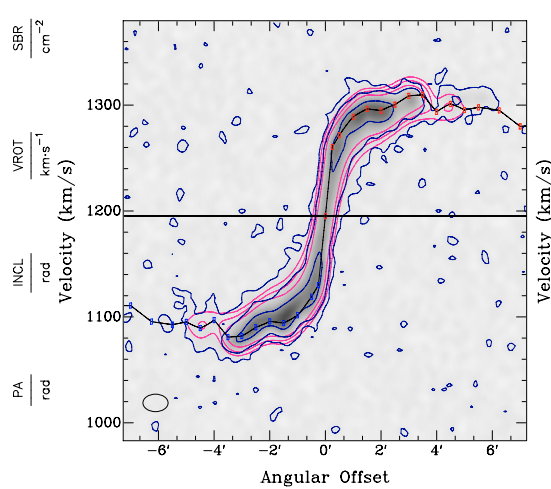

3b)

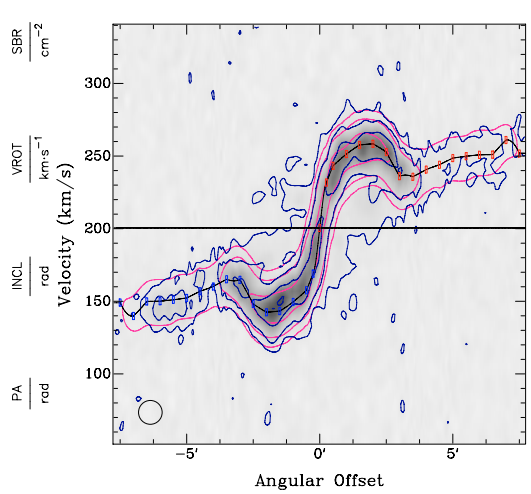

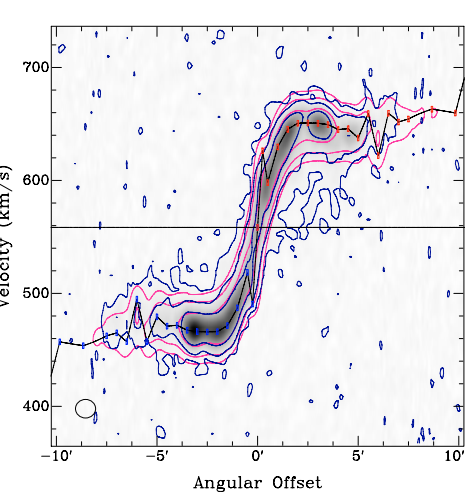

2c)



$3 c)$

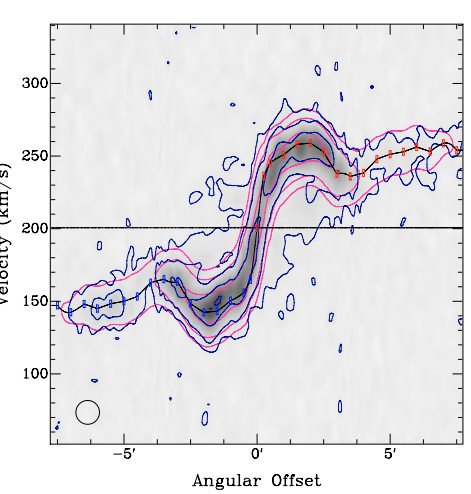

Fig. 12. Comparison of fits to the low-resolution data cube selecting two regions with flat rotation curves. Two fits to the data cube were performed: a) shows the fitting results for the parameters variable with radius, $\mathbf{b}$ ) and $\mathbf{c}$ ) are comparisons of PV diagrams of the original data cube and model data cube along the kinematical major axis. The ellipses in the lower left corner indicate the major axis HPBW and the velocity resolution (FWHM). b) and the blue curve in plot a): Enforcing the two selected regions to have the same rotation velocity. c) and the red curve in plot a): Enforcing the rotation curve to be flat inside the selected regions but allowing the rotation velocity to vary from region to region. Black curve in a): Low-resolution best-fit rotation curve 1) NGC 2541. Contours at 1, 4, 16 mJy/beam. 2) UGC 3580. Contours at 1, 4, 16 mJy/beam. 3) NGC 5204. Contours at $1,4,16,32 \mathrm{mJy} /$ beam. 
G. I. G. Józsa: Kinematic modelling of disk galaxies. II., Online Material p 20
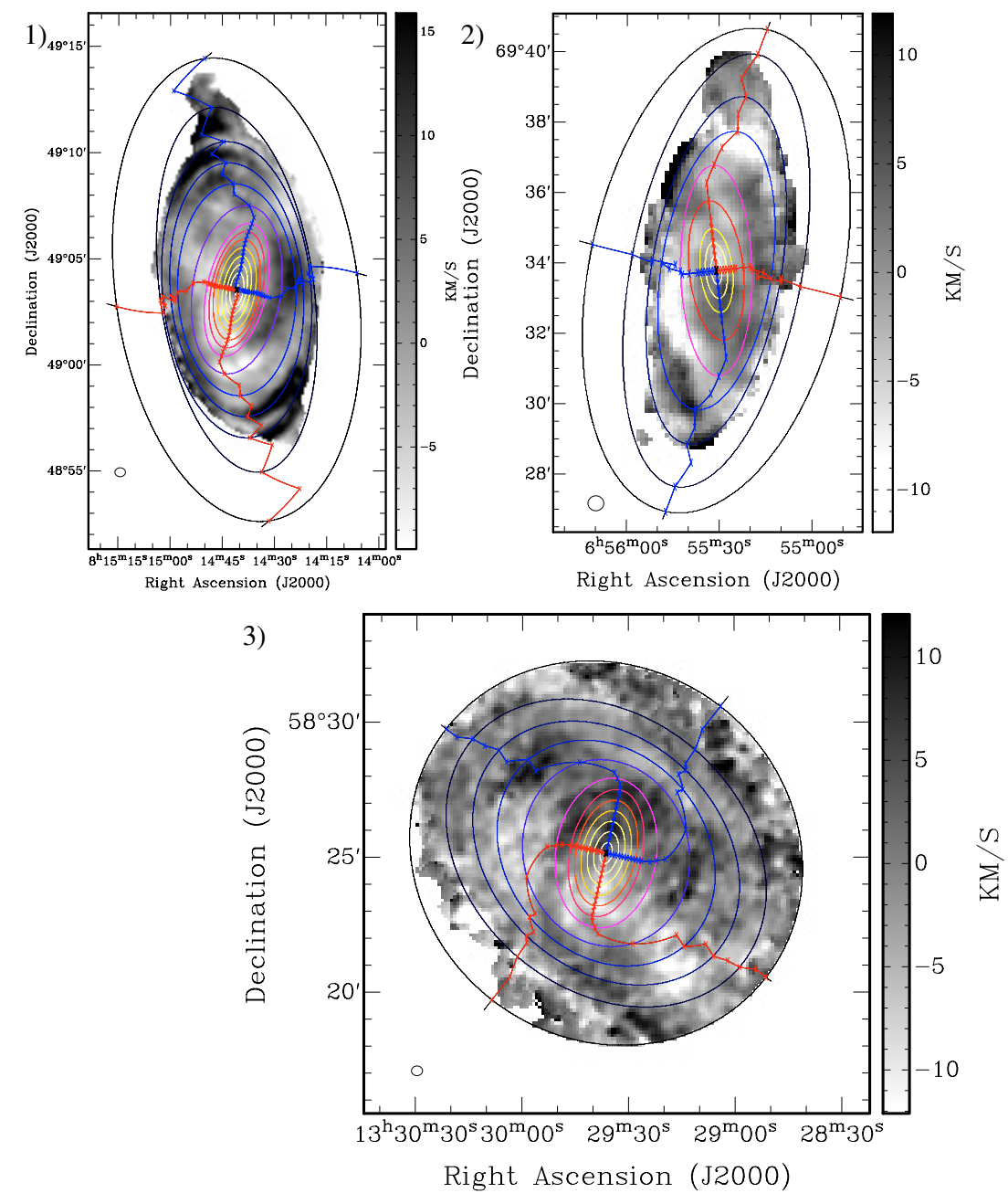

Fig. 13. Difference of velocity maps derived by subtracting the model first moment map from the original first moment map. 1) NGC 2541, 2) UGC 3580, 3) NGC 5204. 